\title{
Elements of Regiocontrol in Palladium-Catalyzed Oxidative Arene Cross-Coupling Reactions
}

\author{
David R. Stuart, Elisia Villemure and Keith Fagnou* \\ Center for Catalysis Research and Innovation, University of Ottawa, Department \\ of Chemistry, 10 Marie Curie, Ottawa Ontario, Canada, K1N 6N5 \\ keith.fagnou@uottawa.ca
}

\section{Supporting Information}

\section{General Methods:}

All indoles and pyrroles were purchased from Aldrich (with the exception of 6-methoxyindole and 6-methylindole, both purchased from Alfa Aesar) and used without further purification. HPLC grade benzene and dichloromethane were dried and purified via an MBraun SP Series solvent purification system. Dichloroethane was dried over $\mathrm{CaH}_{2}$; triethylamine was dried over $\mathrm{NaOH}$ and both were freshly distilled before every use. All reactions were performed in airdried or oven-dried glassware. Coupling reactions were performed without regard for exclusion of ambient air or moisture (specific conditions described below). Analysis of crude reaction mixtures were done on an Agilent 6890 Network GC System with an Agilent 5973 Network Mass Selective Detector. Reactions were purified by flash chromatography or Chromatotron on silica gel. ${ }^{1} \mathrm{H},{ }^{13} \mathrm{C}$, and DEPT-135 spectra were recorded in $\mathrm{CDCl}_{3}$ solutions on a Bruker AVANCE $400 \mathrm{MHz}$ spectrometer at ambient temperature and chemical shifts are reported relative to tetramethylsilane (TMS). Fourier-transform infra-red (FTIR) spectra were obtained as thin films on sodium chloride plates. High resolution mass spectra were obtained with a Kratos Concept IIH mass spectrometer. Melting points were recorded using a Gallenkamp Melting Point Apparatus and are reported uncorrected.

\section{General procedure for the pivalylation of indoles :}

Indole (2.0345 g, $17.4 \mathrm{mmol}, 1$ eq.) and DMAP (0.2097 g, $1.72 \mathrm{mmol}, 0.1$ eq.) were weighed into a round bottom flask. The flask was purged with argon and dry DCM $(30 \mathrm{~mL})$ was added to yield a clear, colorless solution. Triethylamine $(3.6 \mathrm{~mL}, 25.8 \mathrm{mmol}, 1.48$ eq.) was added and the flask was submerged into an ice-water bath and cooled to $0^{\circ} \mathrm{C}$. Pivaloyl chloride $(2.5 \mathrm{~mL}$, $20.3 \mathrm{mmol}, 1.17$ eq.) was added to the flask slowly (over a period of $1 \mathrm{~min}$.) via syringe and the reaction was allowed to stir at $0^{\circ} \mathrm{C}$ for $\sim 10 \mathrm{~min}$. The reaction was allowed to warm to room temperature and stirred overnight. The following morning the progess of the reaction was checked by tlc. The DCM was removed by rotory evaporation and the residue was partioned between diethyl ether (50 $\mathrm{mL}$ ) and a saturated solution of ammonium chloride $(50 \mathrm{~mL})$. The layers were separated and the etherial layer was washed with brine $(25 \mathrm{~mL})$. The combined 
aqueous layers were back extracted with a further $50 \mathrm{~mL}$ of diethyl ether and the combined organic fractions were dried with $\mathrm{MgSO}_{4}$ and evaporated to dryness. Purification was performed under the specific conditions described below.

\section{$N$-Pivaloylindole :}<smiles>CC(C)(C)C(=O)n1ccc2ccccc21</smiles>

The title compound was prepared according to the above procedure and was subjected to column chromatography on silica gel with $5 \%$ diethyl ether in hexanes as the solvent to afford the title compound in $94 \%$ yield.

${ }^{1} \mathrm{H}$ NMR (400 MHz, $\left.\mathrm{CDCl}_{3}, 293 \mathrm{~K}\right): \delta 8.52(\mathrm{dd}, \mathrm{J}=8.4 \mathrm{~Hz}, \mathrm{~J}=0.7 \mathrm{~Hz}, 1 \mathrm{H}), 7.72$ $(\mathrm{d}, \mathrm{J}=3.8 \mathrm{~Hz}, 1 \mathrm{H}), 7.55(\mathrm{~d}, \mathrm{~J}=8.1 \mathrm{~Hz}, 1 \mathrm{H}), 7.34(\mathrm{ddd}, \mathrm{J}=\mathrm{J}=8.3 \mathrm{~Hz}, \mathrm{~J}=1.2 \mathrm{~Hz}$, $1 \mathrm{H}), 7.26(\mathrm{ddd}, \mathrm{J}=\mathrm{J}=8.3 \mathrm{~Hz}, \mathrm{~J}=1.1 \mathrm{~Hz}, 1 \mathrm{H}), 6.61(\mathrm{~d}, \mathrm{~J}=3.8 \mathrm{~Hz}, 1 \mathrm{H}), 1.51(\mathrm{~s}$, $9 \mathrm{H})$.

${ }^{13} \mathrm{C}$ NMR (100 MHz, $\left.\mathrm{CDCl}_{3}, 293 \mathrm{~K}\right): \delta 177.1$ (C), 136.7 (C), 129.4 (C), 125.6 $(\mathrm{CH}), 125.1(\mathrm{CH}), 123.5(\mathrm{CH}), 120.5(\mathrm{CH}), 117.3(\mathrm{CH}), 108.2(\mathrm{CH}), 41.2(\mathrm{C})$, $28.7\left(\mathrm{CH}_{3}\right)$.

FTIR: $2980,1692,1448,907 \mathrm{~cm}^{-1}$.

HRMS (EI): calculated for $\mathrm{C}_{13} \mathrm{H}_{15} \mathrm{NO}\left(\mathrm{M}^{+}\right)$201.1154; found for $\mathrm{C}_{13} \mathrm{H}_{15} \mathrm{NO}\left(\mathrm{M}^{+}\right)$ 201.1137.

Melting point (diethyl ether/hexanes) $68-70^{\circ} \mathrm{C}$.

$\mathbf{R}_{\mathbf{f}}$ (5\% diethyl ether in hexanes): 0.4 .

\section{N-Pivaloyl-5-methoxyindole :}<smiles>COc1ccc2c(ccn2C(=O)C(C)(C)C)c1</smiles>

The title compound was prepared according to the above procedure and was subjected to column chromatography on silica gel with $5 \%$ diethyl ether in hexanes to $10 \%$ diethyl ether in hexanes as the solvent to afford the title compound in $93 \%$ yield.

${ }^{1} \mathrm{H}$ NMR (400 MHz, $\left.\mathrm{CDCl}_{3}, 293 \mathrm{~K}\right): \delta 8.41(\mathrm{~d}, \mathrm{~J}=9.1 \mathrm{~Hz}, 1 \mathrm{H}), 7.70(\mathrm{~d}, \mathrm{~J}=3.8 \mathrm{~Hz}$, $1 \mathrm{H}), 7.01(\mathrm{~d}, \mathrm{~J}=2.5 \mathrm{~Hz}, 1 \mathrm{H}), 6.95(\mathrm{dd}, \mathrm{J}=9.1 \mathrm{~Hz}, \mathrm{~J}=2.6 \mathrm{~Hz}, 1 \mathrm{H}), 6.54(\mathrm{~d}, \mathrm{~J}=$ $3.8 \mathrm{~Hz}, 1 \mathrm{H}), 3.85(\mathrm{~s}, 3 \mathrm{H}), 1.51(\mathrm{~s}, 9 \mathrm{H})$. 
${ }^{13} \mathrm{C}$ NMR (100 MHz, CDCl 3 , 293 K): $\delta 176.7$ (C), 156.4 (C), 131.5 (C), 130.3 (C), $126.3(\mathrm{CH}), 118.1(\mathrm{CH}), 113.4(\mathrm{CH}), 108.1(\mathrm{CH}), 103.3(\mathrm{CH}), 55.6\left(\mathrm{CH}_{3}\right), 41.1$ (C), $28.8\left(\mathrm{CH}_{3}\right)$.

FTIR: $2981,1689,1473,905 \mathrm{~cm}^{-1}$.

HRMS (EI): calculated for $\mathrm{C}_{14} \mathrm{H}_{17} \mathrm{NO}_{2}\left(\mathrm{M}^{+}\right)$231.1259; found for $\mathrm{C}_{14} \mathrm{H}_{17} \mathrm{NO}_{2}\left(\mathrm{M}^{+}\right)$ 231.1263.

Melting point (diethyl ether/hexanes) $99-100^{\circ} \mathrm{C}$.

$\mathbf{R}_{\mathbf{f}}$ (10\% diethyl ether in hexanes): 0.24 .

\section{N-Pivaloyl-5-chloroindole :}<smiles>CC(C)(C)C(=O)n1ccc2cc(Cl)ccc21</smiles>

The title compound was prepared according to the above procedure and was subjected to column chromatography on silica gel with $5 \%$ diethyl ether in hexanes as the solvent to afford the title compound in $76 \%$ yield.

${ }^{1} \mathrm{H}$ NMR (400 MHz, $\left.\mathrm{CDCl}_{3}, 293 \mathrm{~K}\right): \delta 8.44(\mathrm{~d}, \mathrm{~J}=8.9 \mathrm{~Hz}, 1 \mathrm{H}), 7.76(\mathrm{~d}, \mathrm{~J}=3.8 \mathrm{~Hz}$, $1 \mathrm{H}), 7.52(\mathrm{~d}, \mathrm{~J}=2.2 \mathrm{~Hz}, 1 \mathrm{H}), 7.29(\mathrm{dd}, \mathrm{J}=8.9 \mathrm{~Hz}, \mathrm{~J}=2.2 \mathrm{~Hz}, 1 \mathrm{H}), 6.56(\mathrm{~d}, \mathrm{~J}=$ $3.8 \mathrm{~Hz}, 1 \mathrm{H}), 1.52(\mathrm{~s}, 9 \mathrm{H})$.

${ }^{13} \mathrm{C}$ NMR (100 MHz, $\left.\mathrm{CDCl}_{3}, 293 \mathrm{~K}\right): \delta 177.0$ (C), 135.1 (C), 130.6 (C), 129.0 (C), $126.8(\mathrm{CH}), 125.2(\mathrm{CH}), 120.1(\mathrm{CH}), 118.3(\mathrm{CH}), 107.5(\mathrm{CH}), 41.3(\mathrm{C}), 28.7$ $\left(\mathrm{CH}_{3}\right)$.

FTIR: $2973,1690,1493,905 \mathrm{~cm}^{-1}$.

HRMS (EI): calculated for $\mathrm{C}_{13} \mathrm{H}_{14} \mathrm{NOCl}\left(\mathrm{M}^{+}\right)$235.0764; found for $\mathrm{C}_{13} \mathrm{H}_{14} \mathrm{NOCl}\left(\mathrm{M}^{+}\right)$ 235.0757.

Melting point (diethyl ether/hexanes) $106-107^{\circ} \mathrm{C}$.

$\mathbf{R}_{\mathbf{f}}$ (5\% diethyl ether in hexanes): 0.28 .

\section{N-Pivaloyl-6-methoxyindole :}<smiles>COc1ccc2ccn(C(=O)C(C)(C)C)c2c1</smiles>

The title compound was prepared according to the above procedure and was subjected to column chromatography on silica gel with $10 \%$ diethyl ether in hexanes as the solvent to afford the title compound in $78 \%$ yield.

${ }^{1} \mathrm{H}$ NMR (400 MHz, $\left.\mathrm{CDCl}_{3}, 293 \mathrm{~K}\right): \delta 8.16(\mathrm{~d}, \mathrm{~J}=2.4 \mathrm{~Hz}, 1 \mathrm{H}), 7.62(\mathrm{~d}, \mathrm{~J}=3.8 \mathrm{~Hz}$, $1 \mathrm{H}), 7.41(\mathrm{~d}, \mathrm{~J}=8.5 \mathrm{~Hz}, 1 \mathrm{H}), 6.91(\mathrm{dd}, \mathrm{J}=8.5 \mathrm{~Hz}, \mathrm{~J}=2.5 \mathrm{~Hz}, 1 \mathrm{H}), 6.54(\mathrm{~d}, \mathrm{~J}=$ $3.8 \mathrm{~Hz}, 1 \mathrm{H}), 3.88(\mathrm{~s}, 3 \mathrm{H}), 1.51(\mathrm{~s}, 9 \mathrm{H})$. 
${ }^{13} \mathrm{C}$ NMR (100 MHz, $\left.\mathrm{CDCl}_{3}, 293 \mathrm{~K}\right): \delta 177.4$ (C), 158.4 (C), 137.7 (C), 124.4 $(\mathrm{CH}), 123.1(\mathrm{C}), 120.8(\mathrm{CH}), 113.2(\mathrm{CH}), 108.2(\mathrm{CH}), 101.2(\mathrm{CH}), 55.6\left(\mathrm{CH}_{3}\right)$, $41.3(\mathrm{C}), 28.7\left(\mathrm{CH}_{3}\right)$.

FTIR: $2980,1692,1310,903.804 \mathrm{~cm}^{-1}$.

HRMS (EI): calculated for $\mathrm{C}_{14} \mathrm{H}_{17} \mathrm{NO}_{2}\left(\mathrm{M}^{+}\right)$231.1259; found for $\mathrm{C}_{14} \mathrm{H}_{17} \mathrm{NO}_{2}\left(\mathrm{M}^{+}\right)$ 231.1249.

Melting point (diethyl ether/hexanes) $110-112^{\circ} \mathrm{C}$.

$\mathbf{R}_{\mathbf{f}}(10 \%$ diethyl ether in hexanes): 0.39 .

\section{N-Pivaloyl-6-methylindole :}<smiles>Cc1ccc2ccn(C(=O)C(C)(C)C)c2c1</smiles>

The title compound was prepared according to the above procedure and was subjected to column chromatography on silica gel with $2 \%$ diethyl ether in hexanes as the solvent to afford the title compound in $72 \%$ yield.

${ }^{1}{ }_{\mathrm{H}}$ NMR $\left(400 \mathrm{MHz}, \mathrm{CDCl}_{3}, 293 \mathrm{~K}\right): \delta 8.38(\mathrm{~d}, \mathrm{~J}=0.7 \mathrm{~Hz}, 1 \mathrm{H}), 7.65(\mathrm{~d}, \mathrm{~J}=3.8 \mathrm{~Hz}$, $1 \mathrm{H}), 7.42(\mathrm{~d}, \mathrm{~J}=7.9 \mathrm{~Hz}, 1 \mathrm{H}), 7.09(\mathrm{dd}, \mathrm{J}=7.9 \mathrm{~Hz}, \mathrm{~J}=0.9 \mathrm{~Hz}, 1 \mathrm{H}), 6.56(\mathrm{~d}, \mathrm{~J}=$ $3.8 \mathrm{~Hz}, 1 \mathrm{H}), 2.48(\mathrm{~s}, 3 \mathrm{H}), 1.50(\mathrm{~s}, 9 \mathrm{H})$.

${ }^{13} \mathrm{C}$ NMR (100 MHz, $\left.\mathrm{CDCl}_{3}, 293 \mathrm{~K}\right): \delta 177.2$ (C), 137.1 (C), 135.2 (C), 127.1 (C), $125.1(\mathrm{CH}), 124.9(\mathrm{CH}), 120.0(\mathrm{CH}), 117.6(\mathrm{CH}), 108.2(\mathrm{CH}), 41.2(\mathrm{C}), 28.7$ $\left(\mathrm{CH}_{3}\right), 22.0\left(\mathrm{CH}_{3}\right)$.

FTIR: $2980,1692,1310,903.804 \mathrm{~cm}^{-1}$.

HRMS (EI): calculated for $\mathrm{C}_{14} \mathrm{H}_{17} \mathrm{NO}_{1}\left(\mathrm{M}^{+}\right)$215.1310; found for $\mathrm{C}_{14} \mathrm{H}_{17} \mathrm{NO}_{1}\left(\mathrm{M}^{+}\right)$ 215.1312.

Melting point (diethyl ether/hexanes) $63-65^{\circ} \mathrm{C}$.

$\mathbf{R}_{\mathbf{f}}(2 \%$ diethyl ether in hexanes): 0.27 .

Methyl 1-(pivaloyl)-1H-indole-6-carboxylate :<smiles>COC(=O)c1ccc2ccn(C(=O)C(C)(C)C)c2c1</smiles>

The title compound was prepared according to the above procedure and was subjected to column chromatography on silica gel with $10 \%$ diethyl ether in hexanes as the solvent to afford the title compound in $87 \%$ yield.

${ }^{1} \mathrm{H}$ NMR (400 MHz, $\left.\mathrm{CDCl}_{3}, 293 \mathrm{~K}\right): \delta 9.21(\mathrm{~s}, 1 \mathrm{H}), 7.98(\mathrm{dd}, \mathrm{J}=8.2 \mathrm{~Hz}, \mathrm{~J}=1.5$ $\mathrm{Hz}, 1 \mathrm{H}), 7.88(\mathrm{~d}, \mathrm{~J}=3.8 \mathrm{~Hz}, 1 \mathrm{H}), 7.59(\mathrm{~d}, \mathrm{~J}=8.2 \mathrm{~Hz}, 1 \mathrm{H}), 6.65(\mathrm{~d}, \mathrm{~J}=3.8 \mathrm{~Hz}$, $1 \mathrm{H}), 3.94(\mathrm{~s}, 3 \mathrm{H}), 1.53(\mathrm{~s}, 9 \mathrm{H})$. 
${ }^{13} \mathrm{C}$ NMR (100 MHz, $\left.\mathrm{CDCl}_{3}, 293 \mathrm{~K}\right): \delta 177.0$ (C), 167.7 (C), $136.2(\mathrm{C}), 133.0$ (C), $128.4(\mathrm{CH}), 126.8(\mathrm{C}), 124.9(\mathrm{CH}), 120.2(\mathrm{CH}), 119.1(\mathrm{CH}), 108.0(\mathrm{CH}), 52.0$ $\left(\mathrm{CH}_{3}\right), 41.4(\mathrm{C}), 28.6\left(\mathrm{CH}_{3}\right)$.

FTIR: 2983, 1717, 904, $780 \mathrm{~cm}^{-1}$.

HRMS (EI): calculated for $\mathrm{C}_{15} \mathrm{H}_{17} \mathrm{NO}_{3}\left(M^{+}\right)$259.1208; found for $\mathrm{C}_{15} \mathrm{H}_{17} \mathrm{NO}_{3}\left(\mathrm{M}^{+}\right)$ 259.1208.

Melting point (diethyl ether/hexanes) $93-94^{\circ} \mathrm{C}$.

$\mathbf{R}_{\mathbf{f}}(10 \%$ diethyl ether in hexanes): 0.19 .

\section{Procedures for the protection of pyrroles:}

\section{N-Pivaloylpyrrole :}<smiles>CC(C)(C)C(=O)n1cccc1</smiles>

Triethylamine ( 1.5 equiv.) was added to a strirring solution of pyrrole (1 equiv.) and trimethylacetyl chloride (1.5 equiv.) in dry DCM $(1.5 \mathrm{M})$ at $0{ }^{\circ} \mathrm{C}$. DMAP ( 0.1 equiv) was added after 5 min. The reaction mixture was then stirred at room temperature until the reaction was complete, after which water was added and the mixture was extracted with 3 portions of EtOAc. The organic layers were combined, dried with $\mathrm{MgSO}_{4}$ and concentrated under reduced pressure. The crude material was purified by silica gel column chromatography with $10 \%$ DCM/hexane as eluent to afford translucid oil ( $71 \%$ yield). The compound was analytically pure as judged by ${ }^{1} \mathrm{H}$ NMR (attached) which matched literature reports (Organic \& Biomolecular Chemistry, 1 (21), 3787-3798, 2003).

\section{1-(1-(Methoxymethyl)-1H-pyrrol-2-yl)ethanone:}<smiles>COCn1cccc1C(C)=O</smiles>

A solution of 2-acetylpyrrole (1 equiv.) in DMF (3.5 M) was added to a suspension of $\mathrm{NaH}$ (1.1 equiv.) in DMF $(2 \mathrm{M})$ at $0{ }^{\circ} \mathrm{C}$. The mixture was stirred for $1 \mathrm{~h}$ at $0{ }^{\circ} \mathrm{C}$ before the addition of chloromethyl methyl ether (1.4 equiv.) at $0{ }^{\circ} \mathrm{C}$. The reaction mixture was then stirred at room temperature until the reaction was complete, after which ice cold water was added and the mixture was extracted with 3 portions of EtOAc. The organic layers were combined, dried with $\mathrm{MgSO}_{4}$ and concentrated under reduced pressure. The crude material was purified by silica gel column chromatography with $10 \%$ acetone/hexane as eluent to afford translucid oil ( $93 \%$ yield). 
${ }^{1} \mathrm{H}$ NMR (400MHz, $\left.\mathrm{CDCl}_{3}, 293 \mathrm{~K}, \mathrm{TMS}\right): \delta 2.45(3 \mathrm{H}, \mathrm{s}), 3.30(3 \mathrm{H}, \mathrm{s}), 5.68(2 \mathrm{H}, \mathrm{s})$, $6.21(1 \mathrm{H}, \mathrm{dd}, \mathrm{J}=2.6,3.9 \mathrm{~Hz}), 7.01(1 \mathrm{H}, \mathrm{dd}, \mathrm{J}=1.7,3.9 \mathrm{~Hz}), 7.05(1 \mathrm{H}, \mathrm{dd}, \mathrm{J}=1.7$, $2.6 \mathrm{~Hz})$.

${ }^{13} \mathrm{C}$ NMR (100MHz, CDCl $3,293 \mathrm{~K}$, TMS): $\delta 27.3\left(\mathrm{CH}_{3}\right), 56.2\left(\mathrm{CH}_{3}\right), 79.1\left(\mathrm{CH}_{2}\right)$, $108.9(\mathrm{CH}), 121.2(\mathrm{CH}), 130.0(\mathrm{CH}), 130.7(\mathrm{C}), 188.7(\mathrm{C})$.

IR $\left(v_{\max } / \mathrm{cm}^{-1}\right): 1083,1107,1236,1323,1411,1653,2824,2938,3109$.

HRMS calculated for $\mathrm{C}_{8} \mathrm{H}_{11} \mathrm{NO}_{2}\left(\mathrm{M}^{+}\right)$: 153.0790; Found: 153.0787

$\mathbf{R}_{\mathbf{f}}: 0.48$ on silica gel $(20 \%$ Acetone/Hexane)

Methyl 1-(methoxymethyl)-1H-pyrrole-2-carboxylate:<smiles>COCn1cccc1C(=O)OC</smiles>

A solution of methyl 2-pyrrolecarboxylate (1 equiv.) in DMF $(0.6 \mathrm{M})$ was added to a suspension of $\mathrm{NaH}$ (1.2 equiv.) in DMF $(0.7 \mathrm{M})$ at $0{ }^{\circ} \mathrm{C}$. The mixture was stirred for $30 \mathrm{~min}$ at $0{ }^{\circ} \mathrm{C}$ before the addition of chloromethyl methyl ether (1.2 equiv.) at $0{ }^{\circ} \mathrm{C}$. The reaction mixture was then stirred at room temperature until the reaction was complete, after which ice cold water and saturated solution of $\mathrm{NaHCO}_{3}$ were added and the mixture was extracted with 3 portions of EtOAc. The organic layers were combined, dried with $\mathrm{MgSO}_{4}$ and concentrated under reduced pressure. The crude material was purified by silica gel column chromatography with $20 \%$ EtOAc/hexane as eluent to afford translucid oil $(93 \%$ yield).

${ }^{1} \mathrm{H}$ NMR (400MHz, $\left.\mathrm{CDCl}_{3}, 293 \mathrm{~K}, \mathrm{TMS}\right): \delta 3.29(3 \mathrm{H}, \mathrm{s}), 3.83(3 \mathrm{H}, \mathrm{s}), 5.65(2 \mathrm{H}, \mathrm{s})$, $6.20(1 \mathrm{H}, \mathrm{t}, \mathrm{J}=3.3 \mathrm{~Hz}), 7.01(2 \mathrm{H}, \mathrm{d}, \mathrm{J}=3.3 \mathrm{~Hz})$.

${ }^{13} \mathrm{C}$ NMR (100MHz, CDCl 3 , 293K, TMS): $\delta 51.2\left(\mathrm{CH}_{3}\right), 56.1\left(\mathrm{CH}_{3}\right), 78.6\left(\mathrm{CH}_{2}\right)$, $108.8(\mathrm{CH}), 119.2(\mathrm{CH}), 122.2(\mathrm{C}), 128.9(\mathrm{CH}), 161.5(\mathrm{C})$.

IR $\left(v_{\max } / \mathrm{cm}^{-1}\right): 1083,1096,1113,1237,1323,1414,1438,1709,2824,2949$, 3121.

HRMS calculated for $\mathrm{C}_{8} \mathrm{H}_{11} \mathrm{NO}_{3}\left(\mathrm{M}^{+}\right)$: 169.0739; Found: 169.0736

$\mathbf{R}_{\mathbf{f}}: 0.59$ on silica gel ( $30 \%$ EtOAc/Hexane)

\section{1-(Methoxymethyl)-1H-pyrrole-2-carbonitrile:}<smiles>COCn1cccc1C#N</smiles>

A solution of pyrrole-2-carbonitrile (1 equiv.) in DMF (0.6 M) was added to a suspension of $\mathrm{NaH}\left(1.2\right.$ equiv.) in DMF $(0.7 \mathrm{M})$ at $0{ }^{\circ} \mathrm{C}$. The mixture was stirred for $30 \mathrm{~min}$ at $0^{\circ} \mathrm{C}$ before the addition of chloromethyl methyl ether (1.2 equiv.) at $0{ }^{\circ} \mathrm{C}$. The reaction mixture was then stirred at room temperature until the reaction was complete, after which ice cold water and saturated solution of 
$\mathrm{NaHCO}_{3}$ were added and the mixture was extracted with 3 portions of EtOAc. The organic layers were combined, dried with $\mathrm{MgSO}_{4}$ and concentrated under reduced pressure. The crude material was purified by silica gel column chromatography with $20 \%$ Acetone/hexane as eluent to afford translucid oil $(89 \%$ yield).

${ }^{1} \mathrm{H}$ NMR (400MHz, $\left.\mathrm{CDCl}_{3}, 293 \mathrm{~K}, \mathrm{TMS}\right): \delta 3.31(3 \mathrm{H}, \mathrm{s}), 5.32(2 \mathrm{H}, \mathrm{s}), 6.26(1 \mathrm{H}$, $\mathrm{dd}, \mathrm{J}=2.8,3.9 \mathrm{~Hz}), 6.86(1 \mathrm{H}, \mathrm{dd}, \mathrm{J}=1.6,3.9 \mathrm{~Hz}), 7.00(1 \mathrm{H}, \mathrm{dd}, \mathrm{J}=1.6,2.8 \mathrm{~Hz})$.

${ }^{13} \mathrm{C}$ NMR (100MHz, $\left.\mathrm{CDCl}_{3}, 293 \mathrm{~K}, \mathrm{TMS}\right): \delta 56.4\left(\mathrm{CH}_{3}\right), 78.8\left(\mathrm{CH}_{2}\right), 104.3(\mathrm{C})$, $110.5(\mathrm{CH}), 113.3(\mathrm{C}), 121.1(\mathrm{CH}), 126.9(\mathrm{CH})$.

IR $\left(v_{\max } / \mathrm{cm}^{-1}\right): 1042,1102,1167,1196,1300,1410,1473,2220,2826,2935$, 3121.

HRMS calculated for $\mathrm{C}_{7} \mathrm{H}_{8} \mathrm{~N}_{2} \mathrm{O}\left(\mathrm{M}^{+}\right)$: 136.0637; Found: 136.0632

$\mathbf{R}_{\mathbf{f}}: 0.43$ on silica gel ( $30 \%$ Acetone/Hexane)

\section{General procedure for the $\mathrm{C} 2$ arylation of $\mathrm{N}$-pivaloylindoles :}

All coupling reactions were performed on a $0.45 \mathrm{mmol}$ (indole substrate) scale at $0.15 \mathrm{M}$ unless otherwise stated. $\mathrm{Pd}(\mathrm{TFA})_{2}(0.0079 \mathrm{~g}, 0.0237 \mathrm{mmol}, 5$ $\mathrm{mol} \%), N$-pivaloylindole $(0.0923 \mathrm{~g}, 0.45 \mathrm{mmol}, 1 \mathrm{eq}), \operatorname{AgOAc}(0.2291 \mathrm{~g}, 1.37$ $\mathrm{mmol}, 3 \mathrm{eq})$, and PivOH $(0.2788 \mathrm{~g}, 2.73 \mathrm{mmol}, 6 \mathrm{eq})$ were weighed into a $4 \mathrm{~mL}$ glass vial. $2.7 \mathrm{~mL}$ of benzene was added and the vial was sealed with a Teflon lined closed cap. The vial was placed into one of the wells of an aluminum block and heated to $110^{\circ} \mathrm{C}$ for $\sim 3$ hours. The crude reaction mixture was analyzed by GC-MS and showed $>99 \%$ consumption of $\mathrm{N}$-pivaloylindole and and a C2:C3:diarylated product distribution of 25:1:0.7. The crude reaction mixture was filtered over celite (washing with $\mathrm{Et}_{2} \mathrm{O}$ ). The solvent was removed and the residue was dissolved in $\mathrm{Et}_{2} \mathrm{O}(30 \mathrm{~mL})$ and washed with a saturated solution of $\mathrm{Na}_{2} \mathrm{CO}_{3}(3 \times 20 \mathrm{~mL})$. The aqueous layers were back extracted with $\mathrm{Et}_{2} \mathrm{O}(3 \times 20$ $\mathrm{mL}$ ) and the combined organic layers were dried over $\mathrm{MgSO}_{4}$ and concentrated. The crude reactions were purified by Chromatotron on $2-4 \mathrm{~mm}$ silica plates ( $\mathrm{Et}_{2} \mathrm{O} /$ hexanes) (see below for individual compounds).

\section{2-Phenyl-N-pivaloylindole :}

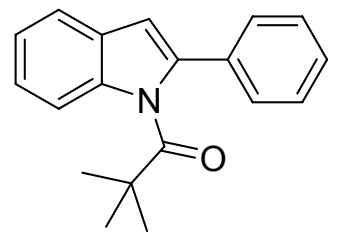

The title compound was prepared according to the general procedure for C2 arylation and was purified by Chromatotron (loaded with DCM) with hexanes $(50 \mathrm{~mL})$ then hexanes/ $\mathrm{Et}_{2} \mathrm{O}(99: 1 ; 200 \mathrm{~mL})$ as the solvent to afford the title compound as an oil in $84 \%$ yield. 
${ }^{1} \mathrm{H}$ NMR (400 MHz, $\left.\mathrm{CDCl}_{3}, 293 \mathrm{~K}\right): \delta 7.62(\mathrm{~d}, \mathrm{~J}=7.3 \mathrm{~Hz}, 1 \mathrm{H}), 7.53(\mathrm{~d}, \mathrm{~J}=7.0 \mathrm{~Hz}$, $2 \mathrm{H}), 7.43(\mathrm{dd}, \mathrm{J}=\mathrm{J}=7.0 \mathrm{~Hz}, 2 \mathrm{H}), 7.38-7.33(\mathrm{~m}, 2 \mathrm{H}), 7.24$ (ddd, $\mathrm{J}=\mathrm{J}=7.2 \mathrm{~Hz}$, $\mathrm{J}=1.4 \mathrm{~Hz}, 1 \mathrm{H}), 7.18(\mathrm{ddd}, \mathrm{J}=\mathrm{J}=7.1 \mathrm{~Hz}, \mathrm{~J}=1.2 \mathrm{~Hz}, 1 \mathrm{H}), 6.68(\mathrm{~s}, 1 \mathrm{H}), 0.96(\mathrm{~s}$, $9 \mathrm{H})$.

${ }^{13} \mathrm{C}$ NMR (100 MHz, CDCl 3 , $\left.293 \mathrm{~K}\right): \delta 187.4$ (C), 139.4 (C), 137.2 (C), 133.9 (C), $129.1(\mathrm{CH}), 128.4(\mathrm{C}), 128.3(\mathrm{CH}), 127.8(\mathrm{CH}), 123.4(\mathrm{CH}), 121.5(\mathrm{CH}), 120.6$ $(\mathrm{CH}), 111.2(\mathrm{CH}), 104.7(\mathrm{CH}) 45.2(\mathrm{C}), 27.9\left(\mathrm{CH}_{3}\right)$.

FTIR: 2969, 1717, 935, 763, $742 \mathrm{~cm}^{-1}$.

HRMS (EI): calculated for $\mathrm{C}_{19} \mathrm{H}_{19} \mathrm{NO}\left(\mathrm{M}^{+}\right)$277.1467; found for $\mathrm{C}_{19} \mathrm{H}_{19} \mathrm{NO}\left(\mathrm{M}^{+}\right)$ 277.1470 .

$\mathbf{R}_{\mathbf{f}}$ (5\% diethyl ether in hexanes): 0.3 .

5-Methoxy-2-phenyl- $N$-pivaloylindole :<smiles>COc1ccc2c(c1)cc(-c1ccccc1)n2C(=O)C(C)(C)C</smiles>

The title compound was prepared according to the general procedure for C2 arylation and was purified by Chromatotron (loaded with DCM) with hexanes $(50 \mathrm{~mL})$ then hexanes/ $\mathrm{Et}_{2} \mathrm{O}(99: 1 ; 200 \mathrm{~mL})$ as the solvent to afford the title compound in $90 \%$ yield.

${ }^{1} \mathrm{H}$ NMR (400 MHz, $\left.\mathrm{CDCl}_{3}, 293 \mathrm{~K}\right): \delta 7.51(\mathrm{~d}, \mathrm{~J}=7.0 \mathrm{~Hz}, 2 \mathrm{H}), 7.43(\mathrm{dd}, \mathrm{J}=\mathrm{J}=$ $7.2 \mathrm{~Hz}, 2 \mathrm{H}), 7.36(\mathrm{dd}, \mathrm{J}=\mathrm{J}=7.3 \mathrm{~Hz}, 1 \mathrm{H}), 7.26(\mathrm{~d}, \mathrm{~J}=8.9 \mathrm{~Hz}, 1 \mathrm{H}), 7.06(\mathrm{~d}, \mathrm{~J}=$ $2.4 \mathrm{~Hz}, 1 \mathrm{H}), 6.89(\mathrm{dd}, \mathrm{J}=8.9 \mathrm{~Hz}, \mathrm{~J}=2.5 \mathrm{~Hz}, 1 \mathrm{H}), 6.61(\mathrm{~s}, 1 \mathrm{H}), 3.86(\mathrm{~s}, 3 \mathrm{H}), 0.95$ (s, 9H).

${ }^{13} \mathrm{C}$ NMR (100 MHz, $\left.\mathrm{CDCl}_{3}, 293 \mathrm{~K}\right): \delta 187.3$ (C), 155.3 (C), 140.1 (C), 134.1 (C), $132.4(\mathrm{CH}), 129.1(\mathrm{C}), 128.9(\mathrm{C}), 128.3(\mathrm{CH}), 127.7(\mathrm{CH}), 113.3(\mathrm{CH}), 112.1$ $(\mathrm{CH}), 104.6(\mathrm{CH}), 102.3(\mathrm{CH}), 55.8\left(\mathrm{CH}_{3}\right), 45.2(\mathrm{C}), 28.0\left(\mathrm{CH}_{3}\right)$.

FTIR: 2968, 1713, 935, 762, $698 \mathrm{~cm}^{-1}$.

HRMS (EI): calculated for $\mathrm{C}_{20} \mathrm{H}_{21} \mathrm{NO}_{2}\left(\mathrm{M}^{+}\right) 307.1572$; found for $\mathrm{C}_{20} \mathrm{H}_{21} \mathrm{NO}_{2}\left(\mathrm{M}^{+}\right)$ 307.1576.

Melting point (diethyl ether/hexanes) $75-76^{\circ} \mathrm{C}$.

$\mathbf{R}_{\mathbf{f}}(10 \%$ diethyl ether in hexanes): 0.41 .

\section{5-Chloro-2-phenyl-N-pivaloylindole :}<smiles>CC(C)(C)C(=O)n1c(-c2ccccc2)cc2cc(Cl)ccc21</smiles> 
The title compound was prepared according to the general procedure for C2 arylation and was purified by Chromatotron (loaded with DCM) with hexanes $(50 \mathrm{~mL})$ then hexanes/ $\mathrm{Et}_{2} \mathrm{O}(99: 1 ; 200 \mathrm{~mL})$ as the solvent to afford the title compound in $86 \%$ yield.

${ }^{1} \mathrm{H}$ NMR (400 MHz, $\left.\mathrm{CDCl}_{3}, 293 \mathrm{~K}\right): \delta 7.58(\mathrm{~d}, \mathrm{~J}=1.8 \mathrm{~Hz}, 1 \mathrm{H}), 7.51(\mathrm{dd}, \mathrm{J}=8.2$ $\mathrm{Hz}, \mathrm{J}=1.6 \mathrm{~Hz}, 2 \mathrm{H}), 7.44(\mathrm{dd}, \mathrm{J}=\mathrm{J}=8.3 \mathrm{~Hz}, 2 \mathrm{H}), 7.38(\mathrm{t}, \mathrm{J}=7.3 \mathrm{~Hz}, 1 \mathrm{H}), 7.26$ $(\mathrm{d}, \mathrm{J}=8.8 \mathrm{~Hz}, 1 \mathrm{H}), 7.19(\mathrm{dd}, \mathrm{J}=8.8 \mathrm{~Hz}, \mathrm{~J}=2.0 \mathrm{~Hz}, 1 \mathrm{H}), 6.61(\mathrm{~s}, 1 \mathrm{H}), 0.94(\mathrm{~s}$, $9 \mathrm{H})$.

${ }^{13} \mathrm{C}$ NMR (100 MHz, CDCl 3 , $\left.293 \mathrm{~K}\right): \delta 187.0$ (C), 140.7 (C), 135.5 (C), 133.4 (C), $129.4(\mathrm{C}), 129.2(\mathrm{CH}), 128.7(\mathrm{CH}), 127.8(\mathrm{CH}), 127.1(\mathrm{C}), 123.6(\mathrm{CH}), 120.0$ $(\mathrm{CH}), 112.2(\mathrm{CH}), 103.9(\mathrm{CH}), 45.2(\mathrm{C}), 27.9\left(\mathrm{CH}_{3}\right)$.

FTIR: 2971, 1720, 917, 762, $695 \mathrm{~cm}^{-1}$.

HRMS (El): calculated for $\mathrm{C}_{19} \mathrm{H}_{18} \mathrm{NOCl}\left(\mathrm{M}^{+}\right) 311.1077$; found for $\mathrm{C}_{19} \mathrm{H}_{18} \mathrm{NOCl}\left(\mathrm{M}^{+}\right)$ 311.1066 .

Melting point (diethyl ether/hexanes) $83-85^{\circ} \mathrm{C}$.

$\mathbf{R}_{\mathbf{f}}$ (1\% diethyl ether in hexanes): 0.3 .

6-Methoxy-2-phenyl-N-pivaloylindole :<smiles>COc1ccc2cc(-c3ccccc3)n(C(=O)C(C)(C)C)c2c1</smiles>

The title compound was prepared according to the general procedure for C2 arylation and was purified by Chromatotron (loaded with DCM) with hexanes $(50 \mathrm{~mL})$, hexanes/ $\mathrm{Et}_{2} \mathrm{O}(99: 1 ; 200 \mathrm{~mL})$, and hexanes/ $\mathrm{Et}_{2} \mathrm{O}(98 / 2 ; 200 \mathrm{~mL})$ as the solvent to afford the title compound in $76 \%$ yield.

${ }^{1} \mathrm{H}$ NMR (400 MHz, $\mathrm{CDCl}_{3}, 293 \mathrm{~K}$ ): $\delta 7.50-7.47(\mathrm{~m}, 3 \mathrm{H}), 7.42$ (dd, $\mathrm{J}=\mathrm{J}=7.6$ $\mathrm{Hz}, 2 \mathrm{H}), 7.33(\mathrm{t}, \mathrm{J}=7.3 \mathrm{~Hz}, 1 \mathrm{H}), 6.87-6.83(\mathrm{~m}, 2 \mathrm{H}), 6.61(\mathrm{~s}, 1 \mathrm{H}), 3.84(\mathrm{~s}, 3 \mathrm{H})$, $0.97(\mathrm{~s}, 9 \mathrm{H})$.

${ }^{13} \mathrm{C}$ NMR (100 MHz, $\left.\mathrm{CDCl}_{3}, 293 \mathrm{~K}\right): \delta 187.5$ (C), 157.5 (C), 138.4 (C), 138.1 (C), $134.2(\mathrm{C}), 129.1(\mathrm{CH}), 127.9(\mathrm{CH}), 127.5(\mathrm{CH}), 122.6(\mathrm{C}), 121.2(\mathrm{CH}), 111.5$ $(\mathrm{CH}), 104.8(\mathrm{CH}), 94.8(\mathrm{CH}), 55.7\left(\mathrm{CH}_{3}\right), 45.3(\mathrm{C}), 28.0\left(\mathrm{CH}_{3}\right)$.

FTIR: 2969, 1717, 932, $757 \mathrm{~cm}^{-1}$.

HRMS (EI): calculated for $\mathrm{C}_{20} \mathrm{H}_{21} \mathrm{NO}_{2}\left(\mathrm{M}^{+}\right) 307.1572$; found for $\mathrm{C}_{20} \mathrm{H}_{21} \mathrm{NO}_{2}\left(\mathrm{M}^{+}\right)$ 307.1566 .

Melting point (diethyl ether/hexanes) $111-113^{\circ} \mathrm{C}$.

$\mathbf{R}_{\mathbf{f}}$ (5\% diethyl ether in hexanes): 0.25 .

\section{6-Methyl-2-phenyl-N-pivaloylindole :}




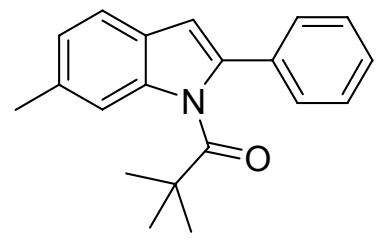

The title compound was prepared according to the general procedure for C2 arylation and was purified by Chromatotron (loaded with DCM) with hexanes $(50 \mathrm{~mL})$ and hexanes/Et ${ }_{2} \mathrm{O}(99: 1 ; 200 \mathrm{~mL})$ as the solvent to afford the title compound in $88 \%$ yield.

${ }^{1} \mathrm{H}$ NMR (400 MHz, $\mathrm{CDCl}_{3}, 293 \mathrm{~K}$ ): $\delta 7.52-7.48(\mathrm{~m}, 3 \mathrm{H}), 7.42(\mathrm{dd}, \mathrm{J}=\mathrm{J}=7.5$ $\mathrm{Hz}, 2 \mathrm{H}), 7.35$ (t, J = 7.3 Hz, 1H), $7.16(\mathrm{~s}, 1 \mathrm{H}), 7.02(\mathrm{~d}, \mathrm{~J}=8.0 \mathrm{~Hz}, 1 \mathrm{H}), 6.63(\mathrm{~s}$, $1 \mathrm{H}), 2.46(\mathrm{~s}, 3 \mathrm{H}), 0.96(\mathrm{~s}, 9 \mathrm{H})$.

${ }^{13} \mathrm{C}$ NMR (100 MHz, $\left.\mathrm{CDCl}_{3}, 293 \mathrm{~K}\right): \delta 187.5$ (C), 138.8 (C), 137.6 (C), 134.2 (C), $133.5(\mathrm{C}), 129.1(\mathrm{CH}), 128.1(\mathrm{CH}), 127.7(\mathrm{CH}), 126.2(\mathrm{C}), 123.3(\mathrm{CH}), 120.2$ $(\mathrm{CH}), 111.1(\mathrm{CH}), 104.7(\mathrm{CH}), 45.1(\mathrm{C}), 28.0\left(\mathrm{CH}_{3}\right), 21.9\left(\mathrm{CH}_{3}\right)$.

FTIR: 2971, 1721, 931, 815, $757 \mathrm{~cm}^{-1}$.

HRMS (EI): calculated for $\mathrm{C}_{20} \mathrm{H}_{21} \mathrm{NO}\left(\mathrm{M}^{+}\right)$291.1623; found for $\mathrm{C}_{20} \mathrm{H}_{21} \mathrm{NO}\left(\mathrm{M}^{+}\right)$ 291.1622.

Melting point (diethyl ether/hexanes) $82-83^{\circ} \mathrm{C}$.

$\mathbf{R}_{\mathbf{f}}$ (1\% diethyl ether in hexanes): 0.26 .

Methyl 2-phenyl-1-(pivaloyl)-1H-indole-6-carboxylate :<smiles>COC(=O)c1ccc2cc(-c3ccccc3)n(C(=O)C(C)(C)C)c2c1</smiles>

The title compound was prepared according to the general procedure for C2 arylation and was purified by Chromatotron (loaded with DCM) with hexanes $(50 \mathrm{~mL})$ and hexanes/ $\mathrm{Et}_{2} \mathrm{O}(95: 5)(300 \mathrm{~mL})$ as the solvent to afford the title compound in $86 \%$ yield.

${ }^{1} \mathrm{H}$ NMR (400 MHz, $\left.\mathrm{CDCl}_{3}, 293 \mathrm{~K}\right): \delta 8.06(\mathrm{~s}, 1 \mathrm{H}), 7.89(\mathrm{dd}, \mathrm{J}=8.3 \mathrm{~Hz}, \mathrm{~J}=1.4$ $\mathrm{Hz}, 1 \mathrm{H}), 7.64(\mathrm{~d}, \mathrm{~J}=8.3 \mathrm{~Hz}, 1 \mathrm{H}), 7.56-7.53(\mathrm{~m}, 2 \mathrm{H}), 7.48-7.38(\mathrm{~m}, 3 \mathrm{H}), 6.72$ (s, 1H), $3.93(\mathrm{~s}, 3 \mathrm{H}), 0.97(\mathrm{~s}, 9 \mathrm{H})$.

${ }^{13} \mathrm{C}$ NMR (100 MHz, CDCl 3 , $\left.293 \mathrm{~K}\right): \delta 186.8$ (C), 167.6 (C), 142.5 (C), 136.5 (C), $133.3(\mathrm{C}), 131.9(\mathrm{C}), 129.2(\mathrm{CH}), 128.9(\mathrm{CH}), 128.0(\mathrm{CH}), 125.0(\mathrm{C}), 122.6(\mathrm{CH})$, $120.2(\mathrm{CH}), 113.3(\mathrm{CH}), 104.4(\mathrm{CH}), 52.0\left(\mathrm{CH}_{3}\right), 45.3(\mathrm{C}), 27.9\left(\mathrm{CH}_{3}\right)$.

FTIR: 2973, 1720, 933, $766 \mathrm{~cm}^{-1}$.

HRMS (EI): calculated for $\mathrm{C}_{21} \mathrm{H}_{21} \mathrm{NO}_{3}\left(\mathrm{M}^{+}\right) 335.1521$; found for $\mathrm{C}_{21} \mathrm{H}_{21} \mathrm{NO}_{3}\left(\mathrm{M}^{+}\right)$ 335.1508.

Melting point (diethyl ether/hexanes) $128-129^{\circ} \mathrm{C}$.

$\mathbf{R}_{\mathbf{f}}$ (5\% diethyl ether in hexanes): 0.12 . 


\section{2-(2',5'-Dimethylbenzene)-N-pivaloylindole :}

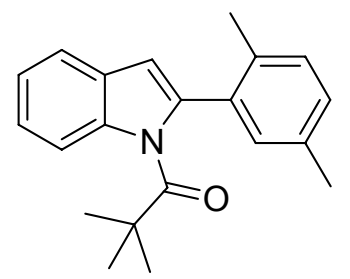

The title compound was prepared according to the following procedure. $\mathrm{Pd}(\mathrm{TFA})_{2}(0.0154 \mathrm{~g}, 0.045 \mathrm{mmol}, 10 \mathrm{~mol} \%), N$-pivaloylindole $(0.0922 \mathrm{~g}, 0.45$ mmol, $1 \mathrm{eq}$ ), AgOAc (0.2373 g, $1.42 \mathrm{mmol}, 3 \mathrm{eq})$, and PivOH (0.2850 g, 2.79 mmol, $6 \mathrm{eq}$ ) were weighed into a $15 \mathrm{~mL}$ sealed tube. $5.5 \mathrm{~mL}$ of $p$-xylene was added and the tube was sealed. The sealed tube was placed into an oil bath and heated to $110^{\circ} \mathrm{C}$ for $\sim 16$ hours. The crude reaction mixture was filtered over celite and washed with diethyl ether. The solvent was removed and the remaining $p$-xylene was removed on a Kugelrohr and the residue was purified by Chromatotron (loaded with DCM) with hexanes $(50 \mathrm{~mL})$ and hexanes: $\mathrm{Et}_{2} \mathrm{O}(99: 1$, $200 \mathrm{~mL}$ ) to afford the title compound as an oil in $58 \%$ yield.

${ }^{1}$ H NMR (400 MHz, $\left.\mathbf{C D C l}_{3}, 293 \mathrm{~K}\right): \delta 7.61(\mathrm{~d}, \mathrm{~J}=8.2 \mathrm{~Hz}, 1 \mathrm{H}), 7.39$ (d, J = $8.2 \mathrm{~Hz}$, $1 \mathrm{H}), 7.25$ (ddd, J = J = 7.6 Hz, J = $1.3 \mathrm{~Hz}, 1 \mathrm{H}), 7.21-7.17(\mathrm{~m}, 2 \mathrm{H}), 7.12-7.07$ (m, 2H), $6.58(\mathrm{~s}, 1 \mathrm{H}), 2.43(\mathrm{~s}, 3 \mathrm{H}), 2.31(\mathrm{~s}, 3 \mathrm{H}), 0.94(\mathrm{~s}, 9 \mathrm{H})$.

${ }^{13} \mathrm{C}$ NMR (100 MHz, $\left.\mathrm{CDCl}_{3}, 293 \mathrm{~K}\right): \delta 186.7$ (C), $137.5(\mathrm{C}), 136.6(\mathrm{C}), 135.5(\mathrm{C})$, $133.4(\mathrm{C}), 132.8(\mathrm{C}), 131.1(\mathrm{CH})$ (two overlapping peaks), $129.1(\mathrm{CH}), 128.3(\mathrm{C})$, $123.3(\mathrm{CH}), 121.5(\mathrm{CH}), 120.4(\mathrm{CH}), 111.5(\mathrm{CH}), 106.7(\mathrm{CH}), 44.4(\mathrm{C}), 27.7$ $\left(\mathrm{CH}_{3}\right), 20.8\left(\mathrm{CH}_{3}\right), 20.1\left(\mathrm{CH}_{3}\right)$.

FTIR: 2969, 1715, 1449, 901, 810, $740 \mathrm{~cm}^{-1}$.

HRMS (EI): calculated for $\mathrm{C}_{21} \mathrm{H}_{23} \mathrm{NO}\left(\mathrm{M}^{+}\right)$305.1780; found for $\mathrm{C}_{21} \mathrm{H}_{23} \mathrm{NO}\left(\mathrm{M}^{+}\right)$ 305.1761 .

$\mathbf{R}_{\mathbf{f}}$ (2\% diethyl ether in hexanes): 0.29.

\section{2-(3',4'-Dimethylbenzene)-N-pivaloylindole :}

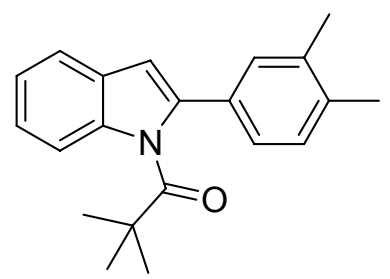

The title compound was prepared according to the general procedure for C2 arylation though was heated at $110^{\circ} \mathrm{C}$ for $\sim 16$ hours and was purified by Chromatotron (loaded with DCM) with hexanes $(250 \mathrm{~mL})$ to afford the title compound as an oily solid in $76 \%$ yield.

${ }^{1} \mathrm{H}$ NMR (400 MHz, $\left.\mathbf{C D C l}_{3}, 293 \mathrm{~K}\right): \delta 7.59(\mathrm{~d}, \mathrm{~J}=7.8 \mathrm{~Hz}, 1 \mathrm{H}), 7.35(\mathrm{~d}, \mathrm{~J}=8.2 \mathrm{~Hz}$, $1 \mathrm{H}), 7.30(\mathrm{~d}, \mathrm{~J}=1.4 \mathrm{~Hz}, 1 \mathrm{H}), 7.26-7.15(\mathrm{~m}, 4 \mathrm{H}), 6.63(\mathrm{~s}, 1 \mathrm{H}), 2.30(\mathrm{~s}, 6 \mathrm{H}), 0.98$ $(\mathrm{s}, 9 \mathrm{H})$. 
${ }^{13} \mathrm{C}$ NMR (100 MHz, $\left.\mathrm{CDCl}_{3}, 293 \mathrm{~K}\right): \delta 187.5$ (C), 139.7 (C), $137.4(\mathrm{C}), 137.1$ (C), $136.9(\mathrm{C}), 131.5(\mathrm{C}), 130.3(\mathrm{CH}), 128.8(\mathrm{CH}), 128.5(\mathrm{C}), 125.2(\mathrm{CH}), 123.1(\mathrm{CH})$, $121.4(\mathrm{CH}), 120.4(\mathrm{CH}), 111.2(\mathrm{CH}), 104.0(\mathrm{CH}), 45.2(\mathrm{C}), 28.0\left(\mathrm{CH}_{3}\right), 19.8$ $\left(\mathrm{CH}_{3}\right), 19.6\left(\mathrm{CH}_{3}\right)$.

FTIR: 2969, 1718, 957, 904, $744 \mathrm{~cm}^{-1}$.

HRMS (EI): calculated for $\mathrm{C}_{21} \mathrm{H}_{23} \mathrm{NO}\left(\mathrm{M}^{+}\right)$305.1780; found for $\mathrm{C}_{21} \mathrm{H}_{23} \mathrm{NO}\left(\mathrm{M}^{+}\right)$ 305.1781 .

$\mathbf{R}_{\mathbf{f}}$ (1\% diethyl ether in hexanes): 0.16 .

\section{3-(3',4'-Dimethylbenzene)- $N$-acetylindole :}<smiles>CC(=O)n1cc(-c2ccc(C)c(C)c2)c2ccccc21</smiles>

The title compound was prepared according to the following procedure. $\mathrm{Pd}(\mathrm{acac})_{2}(0.0176 \mathrm{~g}, 0.06 \mathrm{mmol}, 10 \mathrm{~mol} \%), N$-acetylindole $(0.0950 \mathrm{~g}, 0.60$ $\mathrm{mmol}, 1 \mathrm{eq}), \mathrm{Cu}(\mathrm{OAc})_{2}(0.3340 \mathrm{~g}, 1.8 \mathrm{mmol}, 3 \mathrm{eq})$, and PivOH $(0.3717 \mathrm{~g}, 3.6$ $\mathrm{mmol}, 6 \mathrm{eq}$ ) were weighed into a $4 \mathrm{~mL}$ glass vial. $1.6 \mathrm{~mL}$ of $o$-xylene was added and the vial was sealed with a Teflon-lined cap. The vial was placed into one of the wells of an aluminum block and heated to $110^{\circ} \mathrm{C}$ for $\sim 16$ hours. The crude reaction mixture was filtered over celite and washed with ethyl acetate. The solvent was removed and the remaining o-xylene was removed under reduced pressure. The residue was redissolved in ethyl acetate and extracted with an aqueous solution of ammonium chloride $(2 \times 30 \mathrm{~mL})$, the aqueous extracts were re-extracted with ethyl acetate. The solvent was removed and the residue was purified by flash chromatography with hexanes $(50 \mathrm{~mL})$, hexanes:EtOAc (98:2; $200 \mathrm{~mL})$, hexanes:EtOAc $(95: 5 ; 200 \mathrm{~mL})$ and hexanes $(90: 10 ; 100 \mathrm{~mL})$ to afford the title compound in $61 \%$ yield.

${ }^{1} \mathrm{H}$ NMR (400 MHz, $\left.\mathrm{CDCl}_{3}, 293 \mathrm{~K}\right): \delta 8.51(\mathrm{~d}, \mathrm{~J}=8.1 \mathrm{~Hz}, 1 \mathrm{H}), 7.80(\mathrm{~d}, \mathrm{~J}=7.5 \mathrm{~Hz}$, $1 \mathrm{H}), 7.46(\mathrm{~s}, 1 \mathrm{H}), 7.41-7.37(\mathrm{~m}, 3 \mathrm{H}), 7.33$ (ddd, $\mathrm{J}=\mathrm{J}=7.9 \mathrm{~Hz}, \mathrm{~J}=1.2 \mathrm{~Hz} 1 \mathrm{H}$ ), $7.24(\mathrm{~d}, \mathrm{~J}=7.6 \mathrm{~Hz}, 1 \mathrm{H}), 2.67(\mathrm{~s}, 3 \mathrm{H}), 2.35(\mathrm{~s}, 3 \mathrm{H}), 2.33(\mathrm{~s}, 3 \mathrm{H})$.

${ }^{13} \mathrm{C}$ NMR (100 MHz, CDCl 3 , $\left.293 \mathrm{~K}\right): \delta 168.5$ (C), $137.2(\mathrm{C}), 136.3$ (C), 136.1 (C), $130.8(\mathrm{C}), 130.2(\mathrm{CH}), 129.2(\mathrm{C}), 129.1(\mathrm{CH}), 125.4(\mathrm{C}), 125.4(\mathrm{CH}), 124.1(\mathrm{C})$, $123.8(\mathrm{CH}), 121.6(\mathrm{CH}), 120.0(\mathrm{CH}), 116.8(\mathrm{CH}), 24.1\left(\mathrm{CH}_{3}\right), 19.9\left(\mathrm{CH}_{3}\right), 19.6$ $\left(\mathrm{CH}_{3}\right)$.

FTIR: $2921,1705,1448,747 \mathrm{~cm}^{-1}$.

HRMS (EI): calculated for $\mathrm{C}_{18} \mathrm{H}_{17} \mathrm{NO}\left(\mathrm{M}^{+}\right)$263.1310; found for $\mathrm{C}_{18} \mathrm{H}_{17} \mathrm{NO}\left(\mathrm{M}^{+}\right)$ 263.1315.

Melting point (hexane/ethyl acetate) $123-125^{\circ} \mathrm{C}$.

$\mathbf{R}_{\mathbf{f}}(10 \%$ ethyl acetate in hexanes): 0.22 . 


\section{2-(3',4'-Dichlorobenzene)-N-pivaloylindole :}<smiles>CC(C)(C)C(=O)n1c(-c2ccc(Cl)c(Cl)c2)cc2ccccc21</smiles>

The title compound was prepared according to the general procedure for $\mathrm{C} 2$ arylation though was heated at $110^{\circ} \mathrm{C}$ for $\sim 16$ hours and was purified by Chromatotron (loaded with DCM) with hexanes $(50 \mathrm{~mL})$ and hexanes/ $\mathrm{Et}_{2} \mathrm{O}(99: 1$, $200 \mathrm{~mL}$ ) to afford the title compound in $55 \%$ yield.

${ }^{1} \mathrm{H}$ NMR (400 MHz, $\left.\mathrm{CDCl}_{3}, 293 \mathrm{~K}\right): \delta 7.64-7.61(\mathrm{~m}, 2 \mathrm{H}), 7.51(\mathrm{~d}, \mathrm{~J}=8.3 \mathrm{~Hz}$, $1 \mathrm{H}), 7.36-7.33(\mathrm{~m}, 2 \mathrm{H}), 7.28$ (ddd, $\mathrm{J}=\mathrm{J}=8.2 \mathrm{~Hz}, \mathrm{~J}=1.3,1 \mathrm{H}), 7.20$ (ddd, J = J $=7.9 \mathrm{~Hz}, \mathrm{~J}=1.2 \mathrm{~Hz}, 1 \mathrm{H}), 6.71(\mathrm{~s}, 1 \mathrm{H}), 1.02(\mathrm{~s}, 9 \mathrm{H})$.

${ }^{13} \mathrm{C}$ NMR (100 MHz, $\left.\mathrm{CDCl}_{3}, 293 \mathrm{~K}\right): \delta 186.8$ (C), $137.4(\mathrm{C}), 136.7$ (C), 133.9 (C), $133.4(\mathrm{C}), 132.4(\mathrm{C}), 131.1(\mathrm{CH}), 129.1(\mathrm{CH}), 128.1(\mathrm{C}), 126.7(\mathrm{CH}), 124.1(\mathrm{CH})$, $121.9(\mathrm{CH}), 120.9(\mathrm{CH}), 111.4(\mathrm{CH}), 105.9(\mathrm{CH}), 45.2(\mathrm{C}), 28.0\left(\mathrm{CH}_{3}\right)$.

FTIR: 2972, 1719, 1445, 950, $740 \mathrm{~cm}^{-1}$.

HRMS (EI): calculated for $\mathrm{C}_{19} \mathrm{H}_{17} \mathrm{NOCl}_{2}\left(\mathrm{M}^{+}\right)$345.0687; found for $\mathrm{C}_{19} \mathrm{H}_{17} \mathrm{NOCl}_{2}$ $\left(\mathrm{M}^{+}\right)$345.0696.

Melting point (diethyl ether/hexanes) $132-134^{\circ} \mathrm{C}$.

$\mathbf{R}_{\mathbf{f}}$ (1\% diethyl ether in hexanes): 0.28 .

Procedures for the oxidative arylation of pyrroles:

1-(1-(Methoxymethyl)-5-phenyl-1H-pyrrol-2-yl)ethanone:<smiles>COCn1c(C(C)=O)ccc1-c1ccccc1</smiles>

$\mathrm{Pd}(\mathrm{OPiv})_{2}$ (10 mol\%), 3-nitropyridine (10 mol\%) and AgOAc (3 equiv.) were weighed to air and placed in a screw cap vial. The protected pyrrole (1 equiv.) was added in the vial via a solution of benzene/PivOH (4:1) (0.3 M). The reaction mixture was stirred at $100{ }^{\circ} \mathrm{C}$ for $15-20 \mathrm{~h}$. The crude mixture was filtered on a pad of celite and was eluted with DCM. The GC-MS analysis of the crude mixture showed $71.8 \%$ of monoarylated product, $5.2 \%$ of diarylated product, $1.5 \%$ of homocoupling of the starting pyrrole and $21.5 \%$ of benzene homo coupling. NOESY NMR proved that the regiochemistry of the major regioisomer was the C2 arylated product (see below). Complete conversion was obtained. The volatiles were then removed under reduced pressure and the residue was purified via silica gel column chromatography with $10 \%$ acetone/hexane as eluent to afford a yellow pale oil ( $64 \%$ yield).

Regioselectivity (C2 Major): 15:1 
${ }^{1} \mathrm{H}$ NMR (400MHz, $\left.\mathrm{CDCl}_{3}, 293 \mathrm{~K}, \mathrm{TMS}\right): \delta 2.51(3 \mathrm{H}, \mathrm{s}), 3.35(3 \mathrm{H}, \mathrm{s}), 5.61(2 \mathrm{H}, \mathrm{s})$, $6.29(1 \mathrm{H}, \mathrm{d}, \mathrm{J}=4.0 \mathrm{~Hz}), 7.10(1 \mathrm{H}, \mathrm{d}, \mathrm{J}=4.0 \mathrm{~Hz}), 7.40-7.47(3 \mathrm{H}, \mathrm{m}), 7.54-7.57(2 \mathrm{H}$, $\mathrm{m})$.

${ }^{13} \mathrm{C}$ NMR (100MHz, CDCl 3 , 293K, TMS): $\delta 27.6\left(\mathrm{CH}_{3}\right), 55.7\left(\mathrm{CH}_{3}\right), 75.4\left(\mathrm{CH}_{2}\right)$, $110.1(\mathrm{CH}), 121.3(\mathrm{CH}), 128.5(\mathrm{CH}), 128.6(\mathrm{CH}), 129.5(\mathrm{CH}), 131.4(\mathrm{C}), 131.4$ (C), $143.9(\mathrm{C}), 188.4(\mathrm{C})$.

IR $\left(v_{\max } / \mathbf{c m}^{-1}\right):$ 1092, 1238, 1391, 1460, 1652, 2821, 2939, 3060, 3115.

HRMS calculated for $\mathrm{C}_{14} \mathrm{H}_{15} \mathrm{NO}_{2}\left(\mathrm{M}^{+}\right)$: 229.1103; Found: 229.1095

$\mathbf{R}_{\mathbf{f}}: 0.53$ on silica gel ( $30 \%$ Acetone/Hexane)

NOESY (400MHz, $\left.\mathrm{CDCl}_{3}, 293 \mathrm{~K}, \mathrm{TMS}\right)$ :

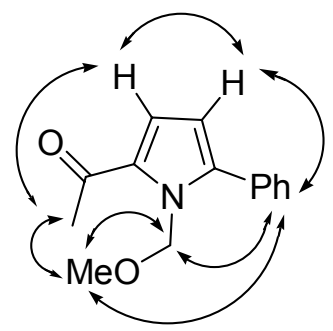

According to the network of the NOESY, the major regioisomer is the C2 arylated product.

\section{1-(1-Methyl-5-phenyl-1H-pyrrol-2-yl)ethanone:}<smiles>CC(=O)c1ccc(-c2ccccc2)n1C</smiles>

$\mathrm{Pd}(\mathrm{OPiv})_{2}$ (10 mol\%), 3-nitropyridine (10 mol\%) and AgOAc (3 equiv.) were weighed to air and placed in a screw cap vial. The protected pyrrole (1 equiv.) was added in the vial via a solution of benzene/PivOH (4:1) $(0.3 \mathrm{M})$. The reaction mixture was stirred at $100{ }^{\circ} \mathrm{C}$ for $15-20 \mathrm{~h}$. The crude mixture was filtered on a pad of celite and was eluted with DCM. The GC-MS analysis of the crude mixture showed $71.2 \%$ of monoarylated product, $4.7 \%$ of diarylated product, $2.1 \%$ of homocoupling of the starting pyrrole and $22.0 \%$ of benzene homo coupling. NOESY NMR proved that the regiochemistry of the major regioisomer was the C2 arylated product (see below). Complete conversion was obtained. The volatiles were then removed under reduced pressure and a saturated solution of $\mathrm{NaHCO}_{3}$ was added. The solution was extracted with 3 portions of $\mathrm{Et}_{2} \mathrm{O}$. The organic layers were combined, dried with $\mathrm{MgSO}_{4}$ and concentrated under reduced pressure. The crude material was then purified by silica gel column chromatography with $10 \%$ Acetone/hexane as eluent to afford a white solid $(68 \%$ yield).

Regioselectivity (C2 Major): 8.5:1

${ }^{1} \mathrm{H}$ NMR (400MHz, CDCl 3 , 293K, TMS): $\delta 2.47(3 \mathrm{H}, \mathrm{s}), 3.88(3 \mathrm{H}, \mathrm{s}), 6.22(1 \mathrm{H}, \mathrm{d}$, $\mathrm{J}=4.1 \mathrm{~Hz}), 7.03(1 \mathrm{H}, \mathrm{d}, \mathrm{J}=4.1 \mathrm{~Hz}), 7.39-7.46(5 \mathrm{H}, \mathrm{m})$. 
${ }^{13} \mathrm{C}$ NMR (100MHz, CDCl 3 , 293K, TMS): $\delta 27.4\left(\mathrm{CH}_{3}\right), 35.2\left(\mathrm{CH}_{3}\right), 109.3(\mathrm{CH})$, $119.7(\mathrm{CH}), 128.3(\mathrm{CH}), 128.5(\mathrm{CH}), 129.4(\mathrm{CH}), 131.8(\mathrm{C}), 131.9(\mathrm{C}), 142.9(\mathrm{C})$, $188.5(\mathrm{C})$.

IR $\left(v_{\max } / \mathrm{cm}^{-1}\right): 1017,1089,1247,1373,1455,1648,2952,2992,3028,3057$, 3106.

HRMS calculated for $\mathrm{C}_{13} \mathrm{H}_{13} \mathrm{NO}\left(\mathrm{M}^{+}\right)$: 199.0997; Found: 199.1000

Melting point $\left({ }^{\circ} \mathrm{C}\right): 69.3-71.0(100 \% \mathrm{DCM})$

$\mathbf{R}_{\mathbf{f}}: 0.53$ on silica gel ( $30 \%$ Acetone/Hexane)

NOESY (400MHz, $\left.\mathrm{CDCl}_{3}, 293 \mathrm{~K}, \mathrm{TMS}\right)$ :

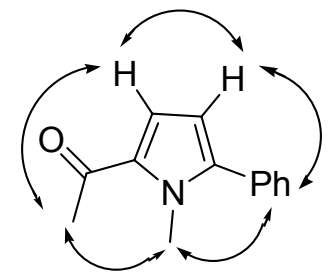

According to the network of the NOESY, the major regioisomer is the C2 arylated product.

Methyl 1-(methoxymethyl)-5-phenyl-1H-pyrrole-2-carboxylate:<smiles>COCn1c(C(=O)OC)ccc1-c1ccccc1</smiles>

$\mathrm{Pd}(\mathrm{OPiv})_{2}(10 \mathrm{~mol} \%)$ and AgOAc (3 equiv.) were weighed to air and placed in a screw cap vial. The protected pyrrole (1 equiv.) was added in the vial via a solution of benzene/PivOH (4:1) (0.3 M). The reaction mixture was stirred at $100{ }^{\circ} \mathrm{C}$ for $3 \mathrm{~h}$. The crude mixture was filtered on a pad of celite and was eluted with DCM. The GC-MS analysis of the crude mixture showed $76.8 \%$ of monoarylated product, $10.2 \%$ of a mixture of diarylated product and homocoupling of the starting pyrrole and $13.0 \%$ of benzene homo coupling. NOESY NMR proved that the regiochemistry of the major regioisomer was the C2 arylated product (see below). Complete conversion was obtained. The volatiles were then removed under reduced pressure and a saturated solution of $\mathrm{NaHCO}_{3}$ was added. The solution was extracted with 3 portions of $\mathrm{Et}_{2} \mathrm{O}$. The organic layers were combined, dried with $\mathrm{MgSO}_{4}$ and concentrated under reduced pressure. The crude material was then purified by silica gel column chromatography with $5 \%$ Acetone/hexane as eluent to afford a beige solid $(66 \%$ yield).

Regioselectivity (C2 Major): 9.8:1

${ }^{1} \mathrm{H}$ NMR (400MHz, CDCl $\left.3,293 \mathrm{~K}, \mathrm{TMS}\right): \delta 3.30(3 \mathrm{H}, \mathrm{s}), 3.85(3 \mathrm{H}, \mathrm{s}), 5.60(2 \mathrm{H}, \mathrm{s})$, 6.26, (1H, d, J=3.9 Hz), $7.09(1 \mathrm{H}, \mathrm{d}, \mathrm{J}=3.9 \mathrm{~Hz}), 7.39-7.55(5 \mathrm{H}, \mathrm{m})$.

${ }^{13} \mathrm{C}$ NMR (100MHz, $\mathrm{CDCl}_{3}$, 293K, TMS): $\delta 51.2\left(\mathrm{CH}_{3}\right), 55.6\left(\mathrm{CH}_{3}\right), 75.1\left(\mathrm{CH}_{2}\right)$, $109.9(\mathrm{CH}), 119.2(\mathrm{CH}), 123.2(\mathrm{C}), 128.3(\mathrm{CH}), 128.6(\mathrm{CH}), 129.5(\mathrm{CH}), 131.8$ (C), $142.7(\mathrm{C}), 161.6(\mathrm{C})$. 
IR $\left(v_{\max } / \mathrm{cm}^{-1}\right): 1107,1162,1240,1276,1330,1462,1707,2827,2949,2994$, 3063

HRMS calculated for $\mathrm{C}_{14} \mathrm{H}_{15} \mathrm{NO}_{3}\left(\mathrm{M}^{+}\right): 245.1052$; Found: 245.1041

Melting point $\left({ }^{\circ} \mathrm{C}\right): 52.9-53.7(100 \% \mathrm{DCM})$

$\mathbf{R}_{\mathbf{f}}$ : 0.55 on silica gel ( $30 \%$ Acetone/Hexane)

NOESY (400MHz, $\left.\mathrm{CDCl}_{3}, 293 \mathrm{~K}, \mathrm{TMS}\right)$ :

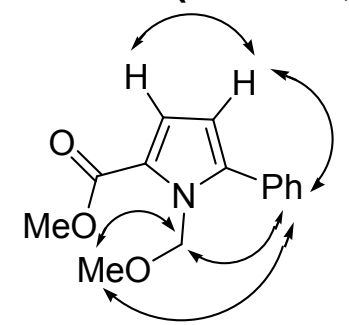

According to the network of the NOESY, the major regioisomer is the C2 arylated product.

\section{1-(Methoxymethyl)-5-phenyl-1H-pyrrole-2-carbonitrile:}<smiles>COCn1c(C#N)ccc1-c1ccccc1</smiles>

$\mathrm{Pd}(\mathrm{OPiv})_{2}(10 \mathrm{~mol} \%)$ and $\mathrm{AgOAc}$ (3 equiv.) were weighed to air and placed in a screw cap vial. The protected pyrrole (1 equiv.) was added in the vial via a solution of benzene/PivOH (4:1) (0.1 M). The reaction mixture was stirred at $100{ }^{\circ} \mathrm{C}$ for $15-20 \mathrm{~h}$. The crude mixture was filtered on a pad of celite and was eluted with DCM. The GC-MS analysis of the crude mixture showed $52.8 \%$ of monoarylated product, $9.5 \%$ of diarylated product and $37.7 \%$ of benzene homo coupling. NOESY NMR proved that the regiochemistry of the major regioisomer was the C2 arylated product (see below). 96\% conversion was obtained. The volatiles were then removed under reduced pressure and a saturated solution of $\mathrm{NaHCO}_{3}$ was added. The solution was extracted with 3 portions of $\mathrm{Et}_{2} \mathrm{O}$. The organic layers were combined, dried with $\mathrm{MgSO}_{4}$ and concentrated under reduced pressure. The crude material was then purified by silica gel column chromatography with $10 \%$ Acetone/hexane as eluent. The regioisomer mixture was repurified by silica gel column chromatography with $10 \% \mathrm{Et}_{2} \mathrm{O} /$ hexane as eluent to afford yellow pale oil (67\% yield).

Regioselectivity (C2 Major): 38.1:1:4.7

${ }^{1} \mathrm{H}$ NMR (400MHz, $\left.\mathrm{CDCl}_{3}, 293 \mathrm{~K}, \mathrm{TMS}\right): \delta 3.44(3 \mathrm{H}, \mathrm{s}), 5.30(2 \mathrm{H}, \mathrm{s}), 6.32(1 \mathrm{H}, \mathrm{d}$, $\mathrm{J}=3.9 \mathrm{~Hz}), 6.92(1 \mathrm{H}, \mathrm{d}, \mathrm{J}=3.9 \mathrm{~Hz}), 7.41-7.47(3 \mathrm{H}, \mathrm{m}), 7.53-7.55(2 \mathrm{H}, \mathrm{m})$.

${ }^{13} \mathrm{C}$ NMR (100MHz, CDCl 3 , 293K, TMS): $\delta 56.5\left(\mathrm{CH}_{3}\right), 76.17\left(\mathrm{CH}_{2}\right), 105.7(\mathrm{C})$, $110.6(\mathrm{CH}), 114.1(\mathrm{C}), 120.8(\mathrm{CH}), 128.7(\mathrm{CH}), 128.8(\mathrm{CH}), 129.2(\mathrm{CH}), 130.8$ (C), $141.0(\mathrm{C})$.

IR $\left(v_{\max } / \mathbf{c m}^{-1}\right): 1091,1132,1188,1311,1391,1461,2217,2825,2936,2991$, 3066, 3134.

HRMS calculated for $\mathrm{C}_{13} \mathrm{H}_{12} \mathrm{~N}_{2} \mathrm{O}\left(\mathrm{M}^{+}\right): 212.0950$; Found: 212.0954 
$\mathbf{R}_{\mathrm{f}}: 0.42$ on silica gel ( $20 \%$ Acetone/Hexane)

NOESY $\left(400 \mathrm{MHz}, \mathrm{CDCl}_{3}, 293 \mathrm{~K}, \mathrm{TMS}\right)$ :

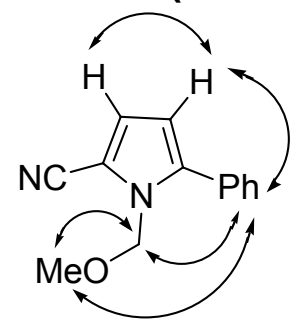

According to the network of the NOESY, the major regioisomer is the C2 arylated product.

\section{2,5-Diphenyl-N-Pivaloylpyrrole :}<smiles>CC1(C)C(=O)n2c(-c3ccccc3)ccc2-c2ccccc21</smiles>

$\mathrm{Pd}(\mathrm{OPiv})_{2}(10 \mathrm{~mol} \%)$ and AgOAc (5 equiv.) were weighed to air and placed in a screw cap vial. The protected pyrrole (1 equiv.) was added in the vial via a solution of benzene/PivOH (4:1) (0.1 M). The reaction mixture was stirred at $100{ }^{\circ} \mathrm{C}$ for $15-20 \mathrm{~h}$. The crude mixture was filtered on a pad of celite and was eluted with DCM. The GC-MS analysis of the crude mixture showed $8.3 \%$ of monoarylated product, $77.7 \%$ of diarylated product and $14.0 \%$ of benzene homo coupling. NOESY NMR proved that the regiochemistry of the major regioisomer was the C2, C5 diarylated product (see below). Complete conversion was obtained. The volatiles were then removed under reduced pressure and a saturated solution of $\mathrm{NaHCO}_{3}$ was added. The solution was extracted with 3 portions of $\mathrm{Et}_{2} \mathrm{O}$. The organic layers were combined, dried with $\mathrm{MgSO}_{4}$ and concentrated under reduced pressure. The crude material was then purified by silica gel column chromatography with $10 \%$ Toluene/hexane as eluent to afford pale red oil ( $53 \%$ yield).

Regioselectivity (C2, C5 Major): 6.1:1

${ }^{1} \mathrm{H}$ NMR (400MHz, $\left.\mathrm{CDCl}_{3}, 293 \mathrm{~K}, \mathrm{TMS}\right): \delta 0.58(9 \mathrm{H}, \mathrm{s}), 6.40(2 \mathrm{H}, \mathrm{s}), 7.25-7.29$ $(2 \mathrm{H}, \mathrm{m}), 7.35-7.38(4 \mathrm{H}, \mathrm{m}), 7.50-7.52(4 \mathrm{H}, \mathrm{m})$.

${ }^{13} \mathrm{C}$ NMR (100MHz, $\left.\mathrm{CDCl}_{3}, 293 \mathrm{~K}, \mathrm{TMS}\right): \delta 26.8\left(\mathrm{CH}_{3}\right), 45.6(\mathrm{C}), 110.1(\mathrm{CH})$, $127.3(\mathrm{CH}), 128.4(\mathrm{CH}), 128.6(\mathrm{CH}), 132.9(\mathrm{C}), 135.2(\mathrm{C}), 189.7(\mathrm{C})$.

IR $\left(v_{\max } / \mathrm{cm}^{-1}\right): 1010,1184,1300,1367,1485,1603,1735,2872,2906,2934$, 2976, 3065.

HRMS calculated for $\mathrm{C}_{21} \mathrm{H}_{21} \mathrm{NO}\left(\mathrm{M}^{+}\right)$: 303.1623; Found: 303.1623

$\mathbf{R}_{\mathbf{f}}: 0.72$ on silica gel ( $30 \%$ Acetone/Hexane)

NOESY (400MHz, $\left.\mathrm{CDCl}_{3}, 293 \mathrm{~K}, \mathrm{TMS}\right)$ : 


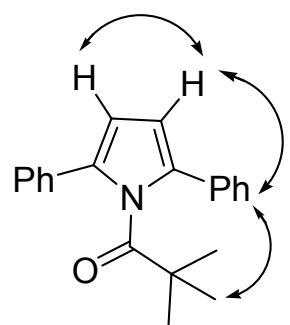

According to the network of the NOESY, the major regioisomer is the $\mathrm{C} 2, \mathrm{C} 5$ diarylated product. 


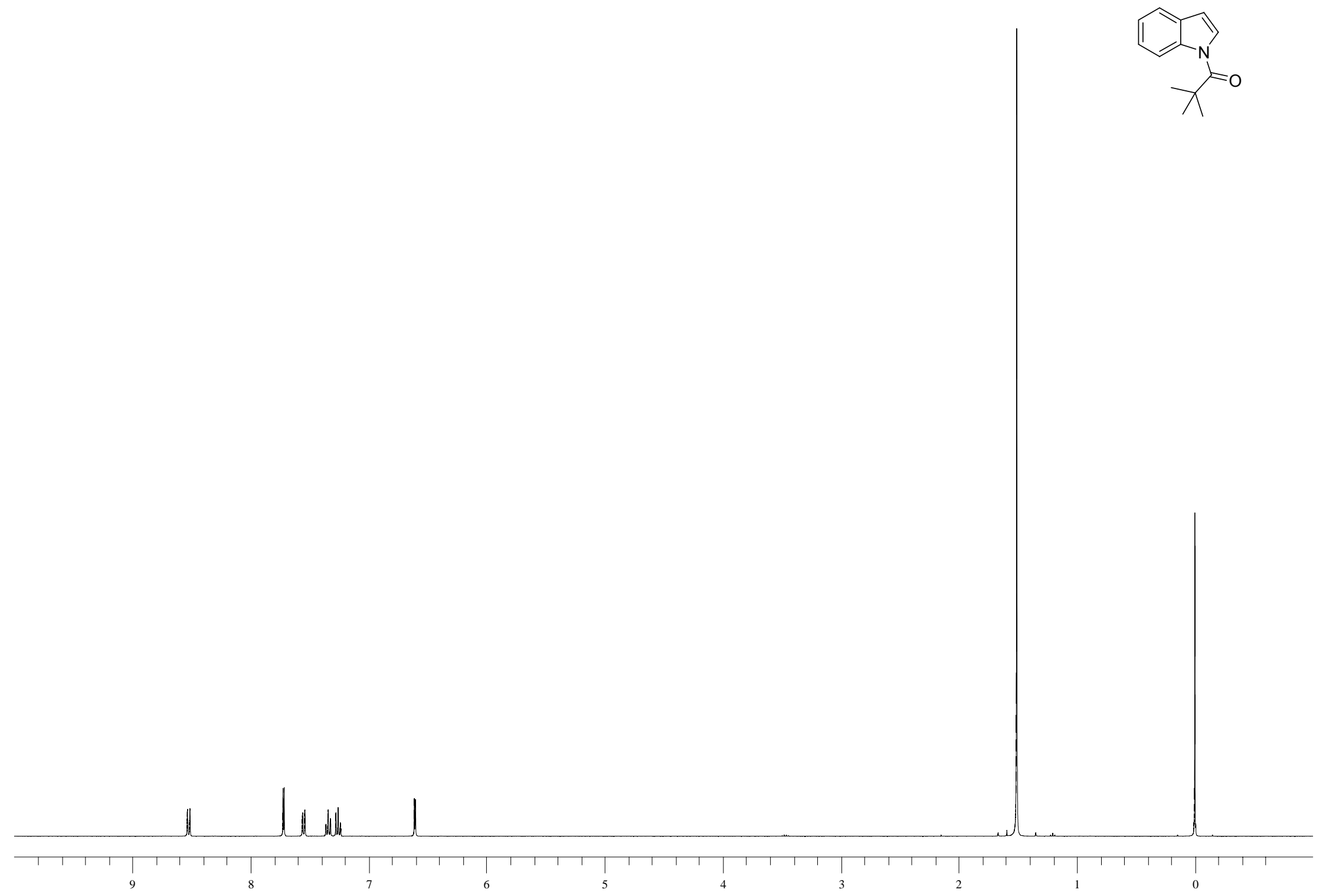


S20
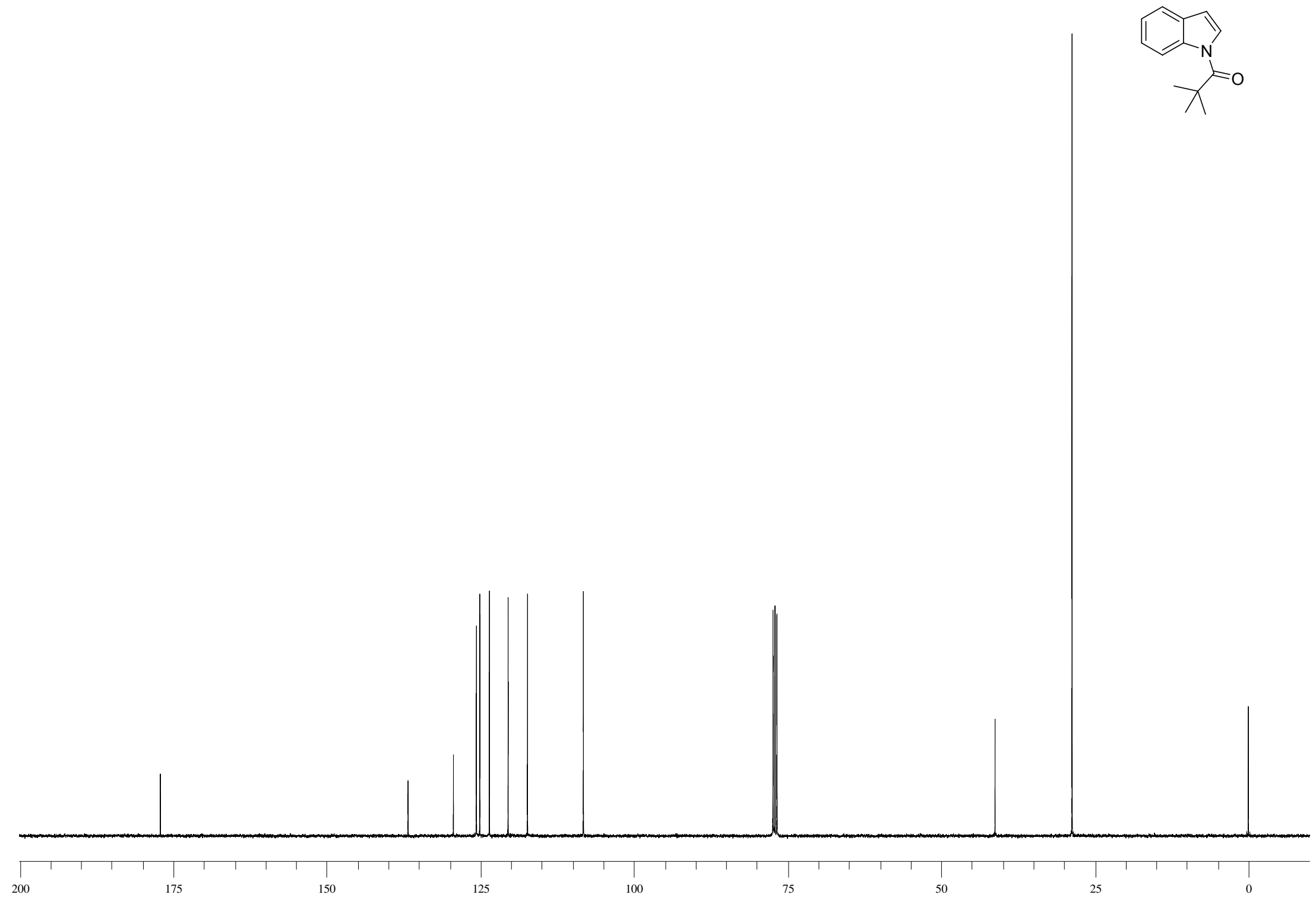
S21

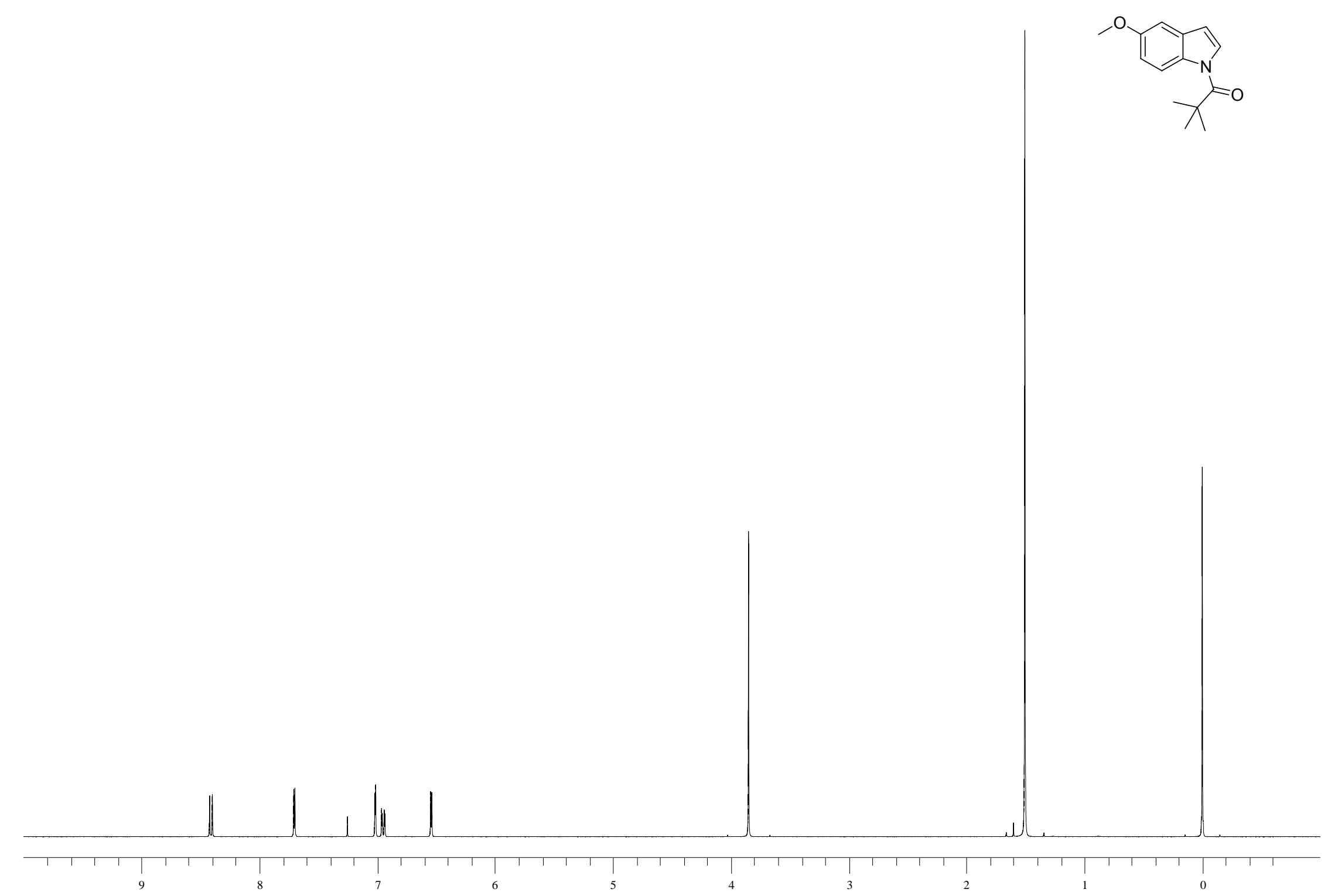




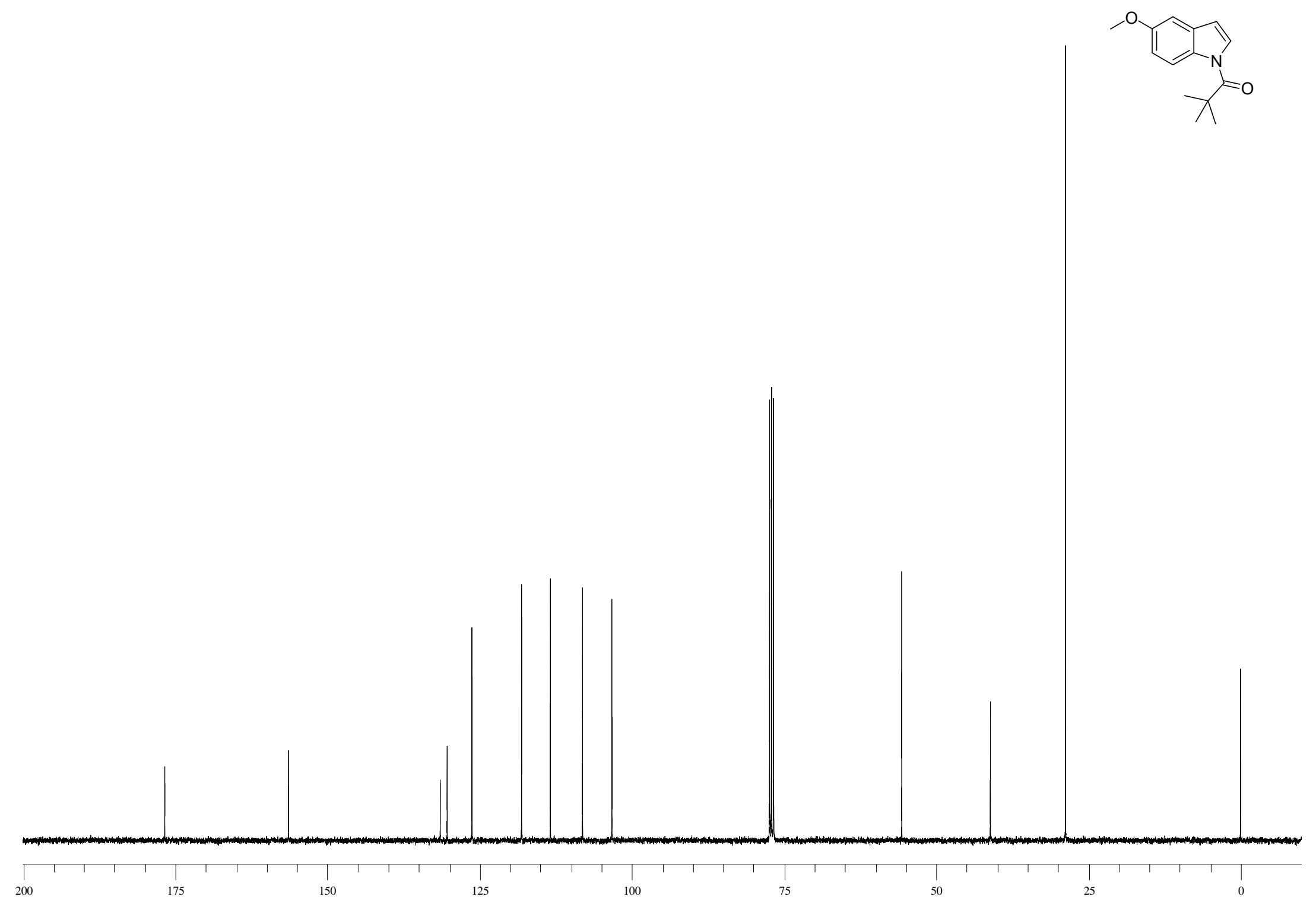


S23
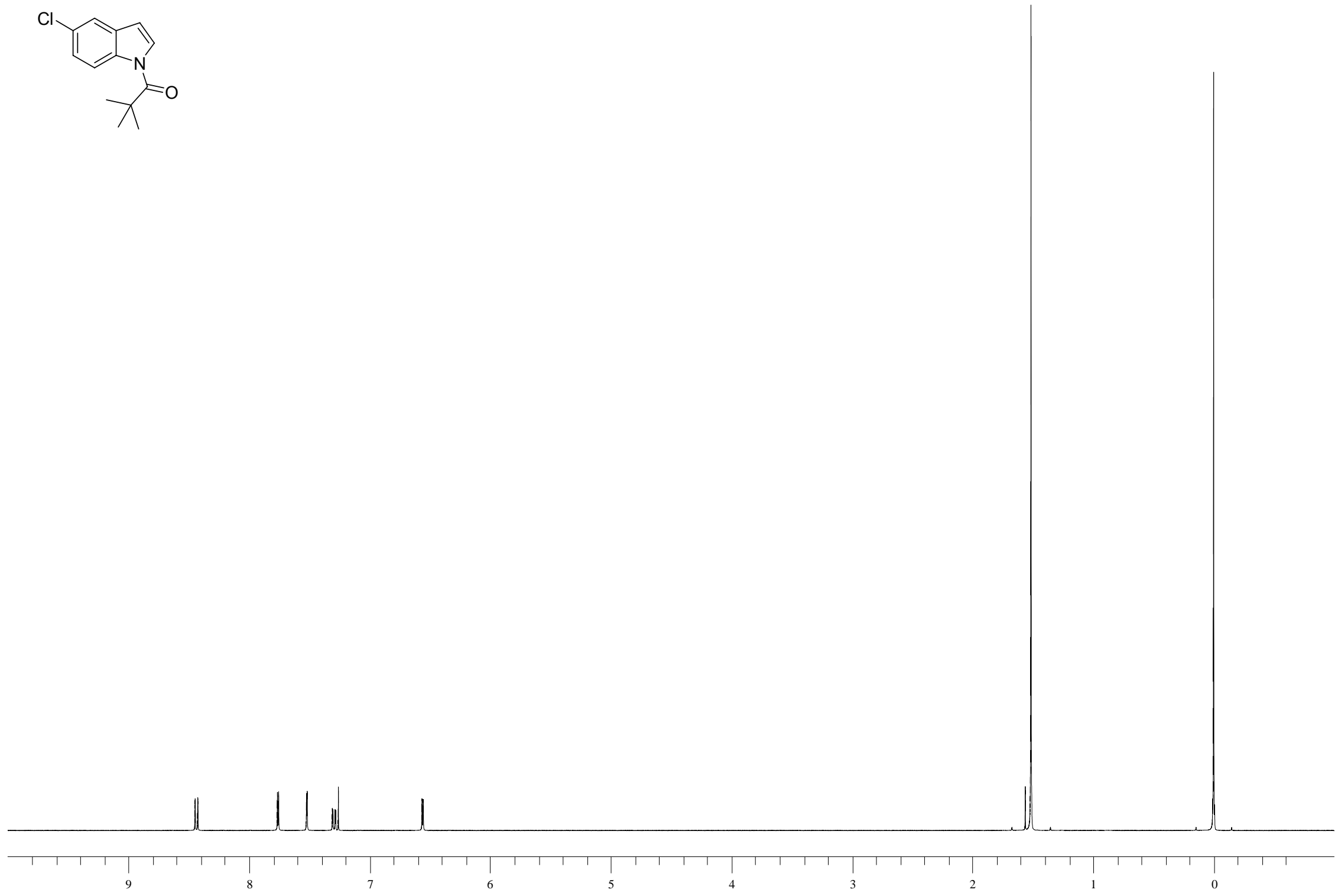


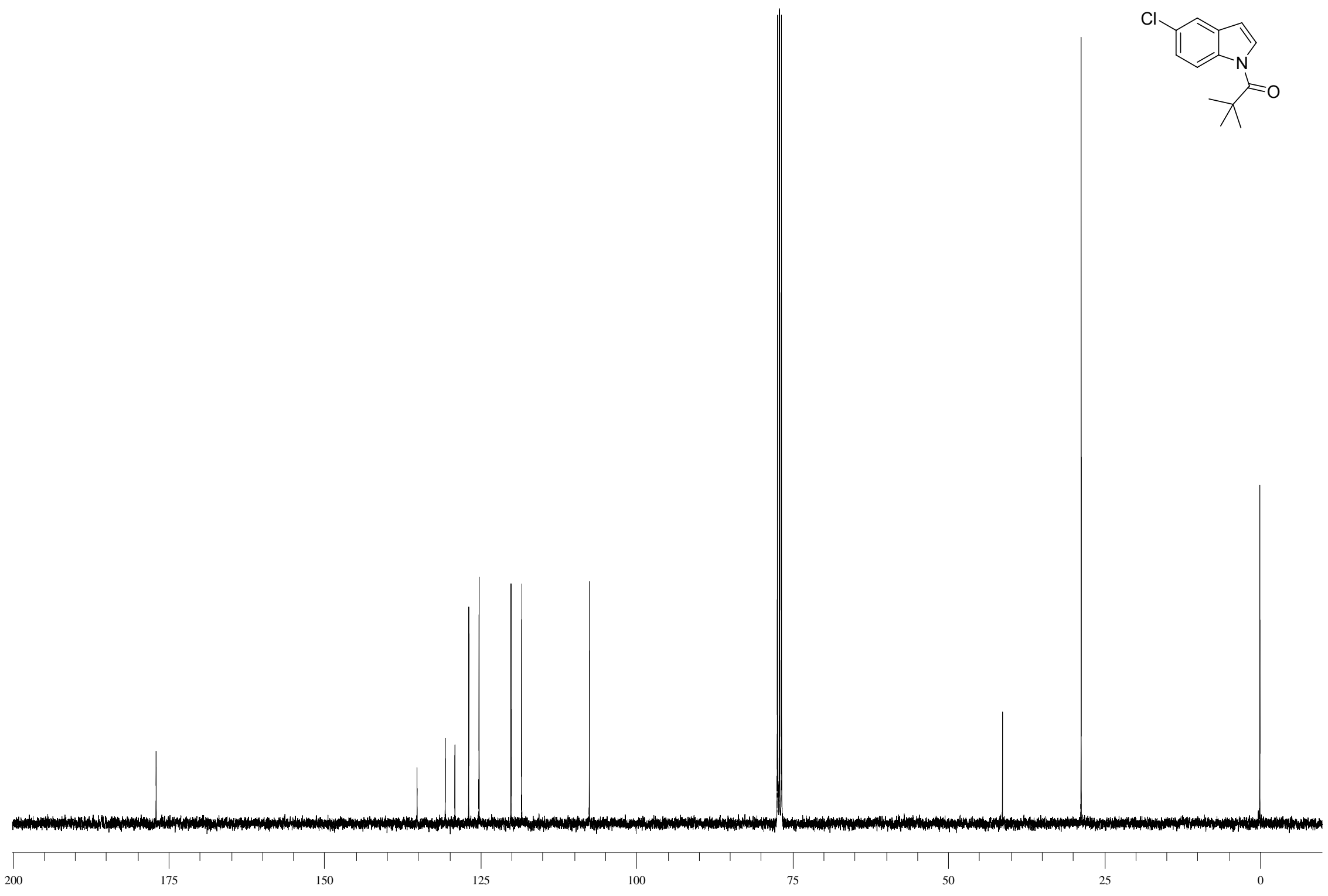




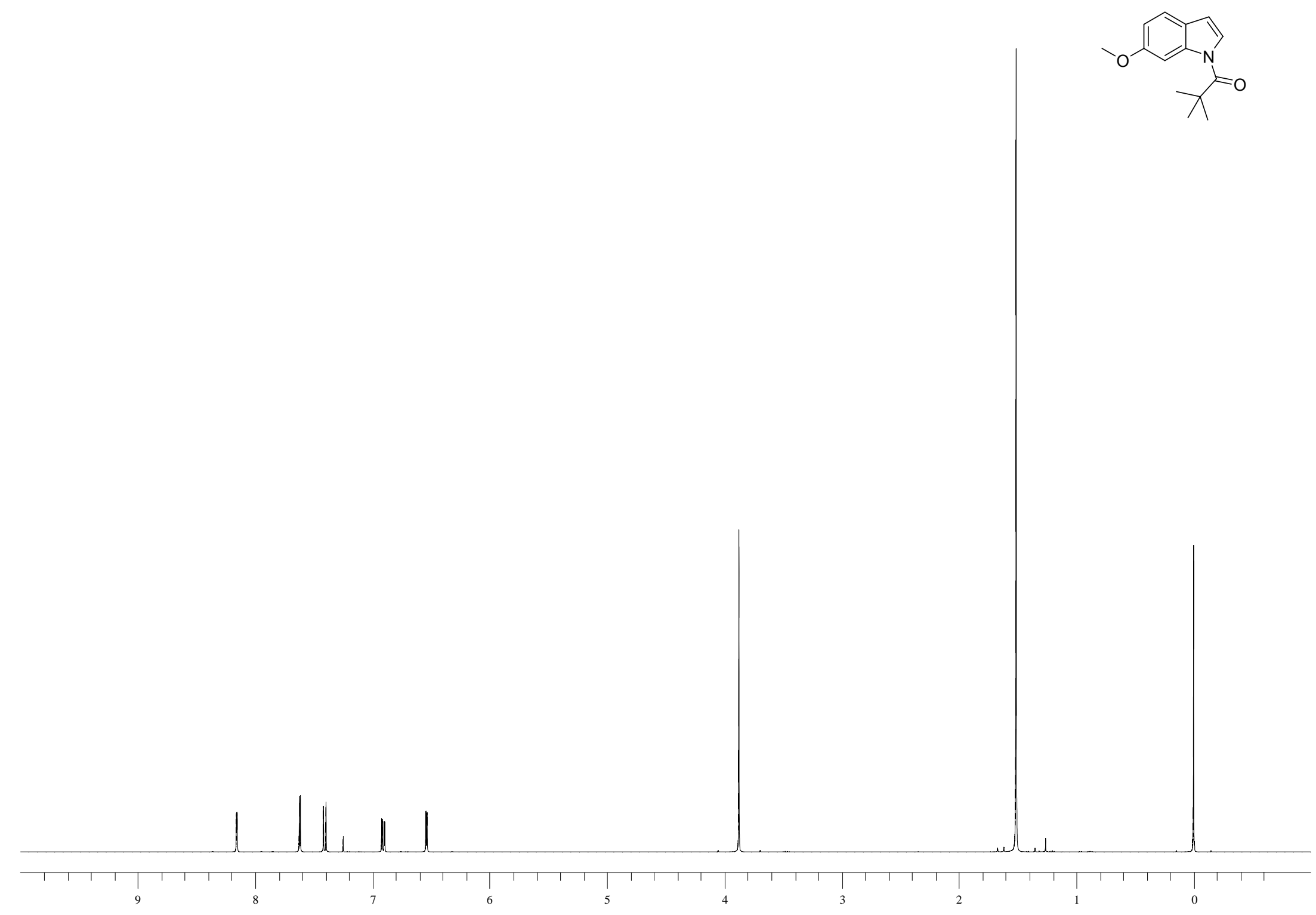




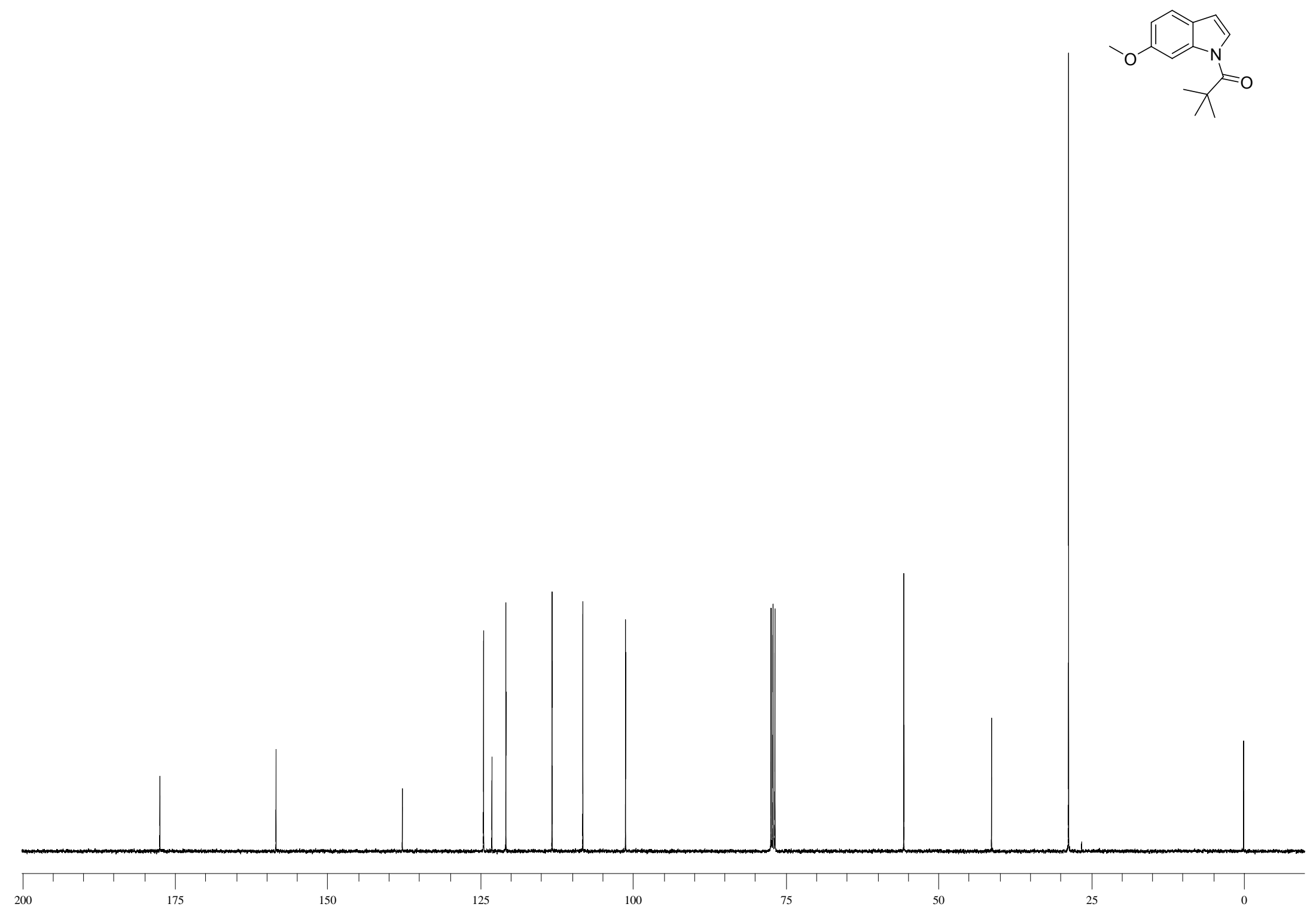




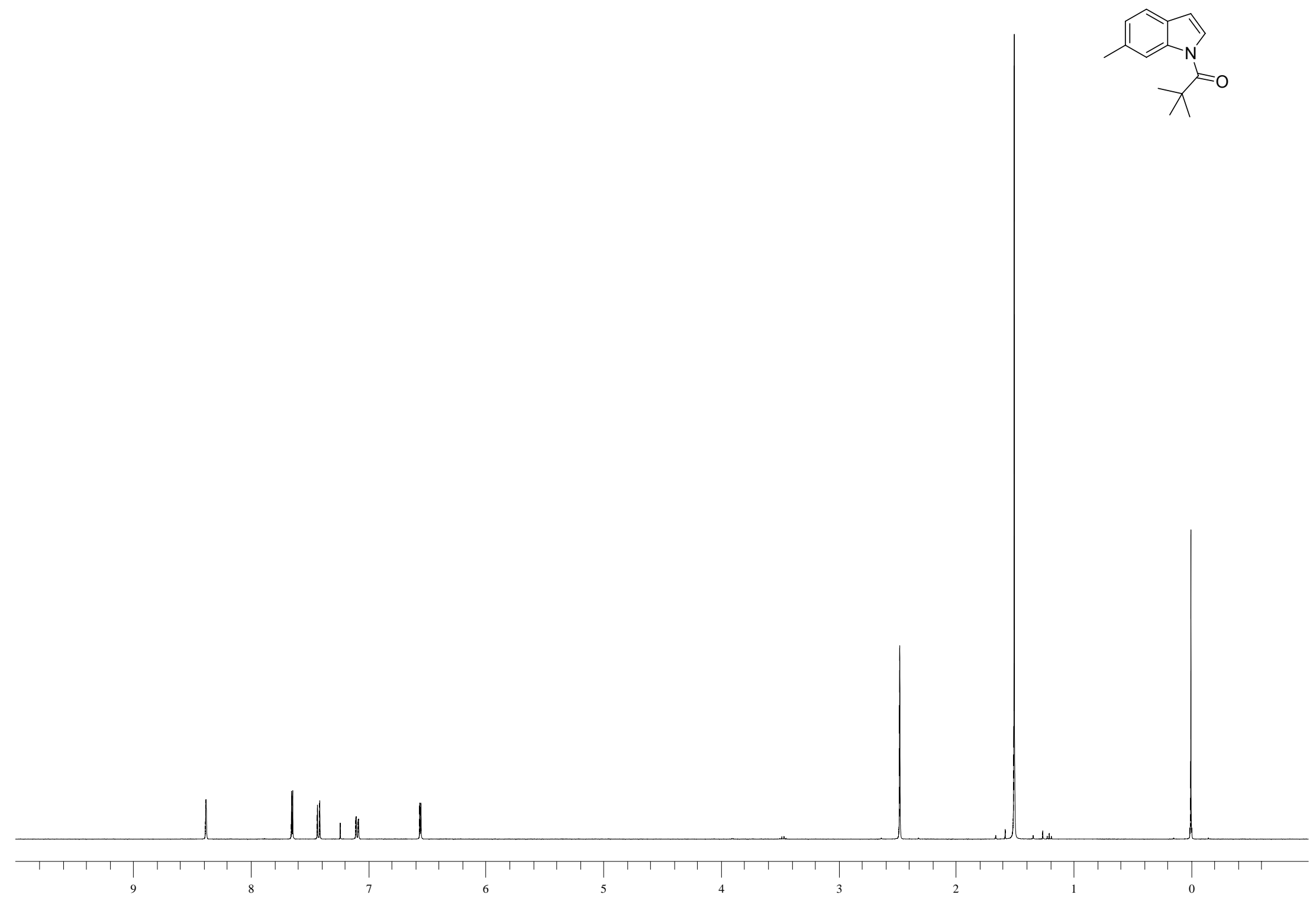


S28

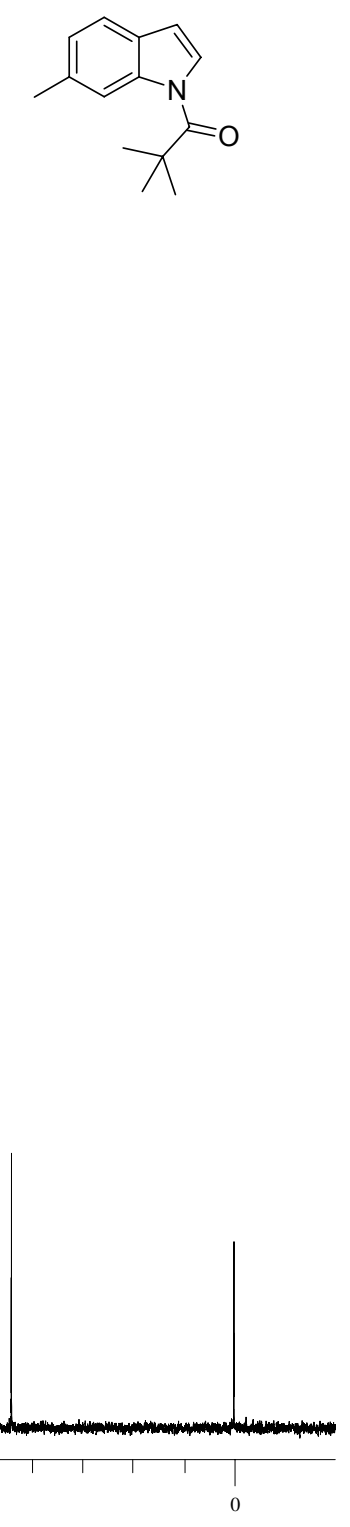




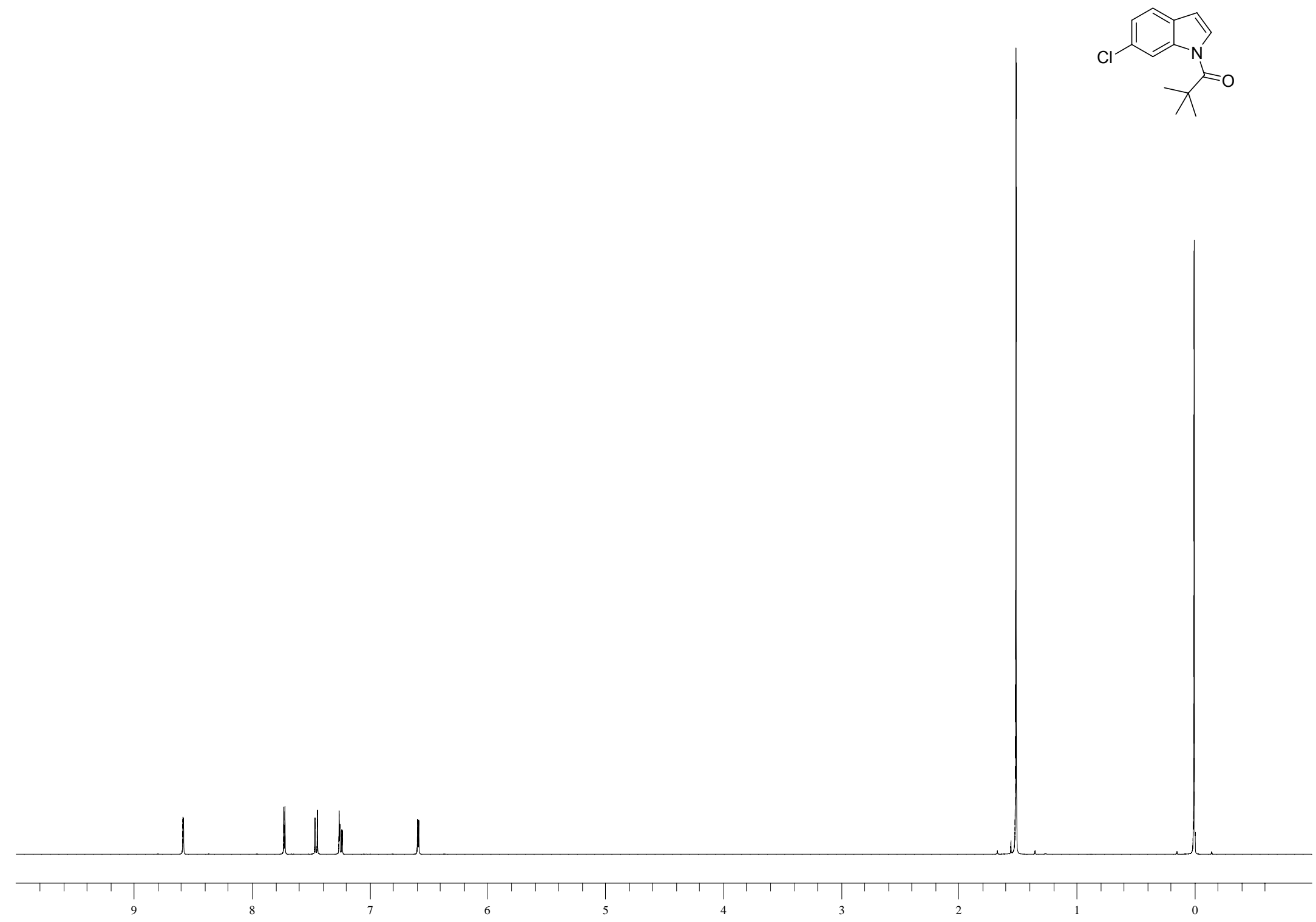




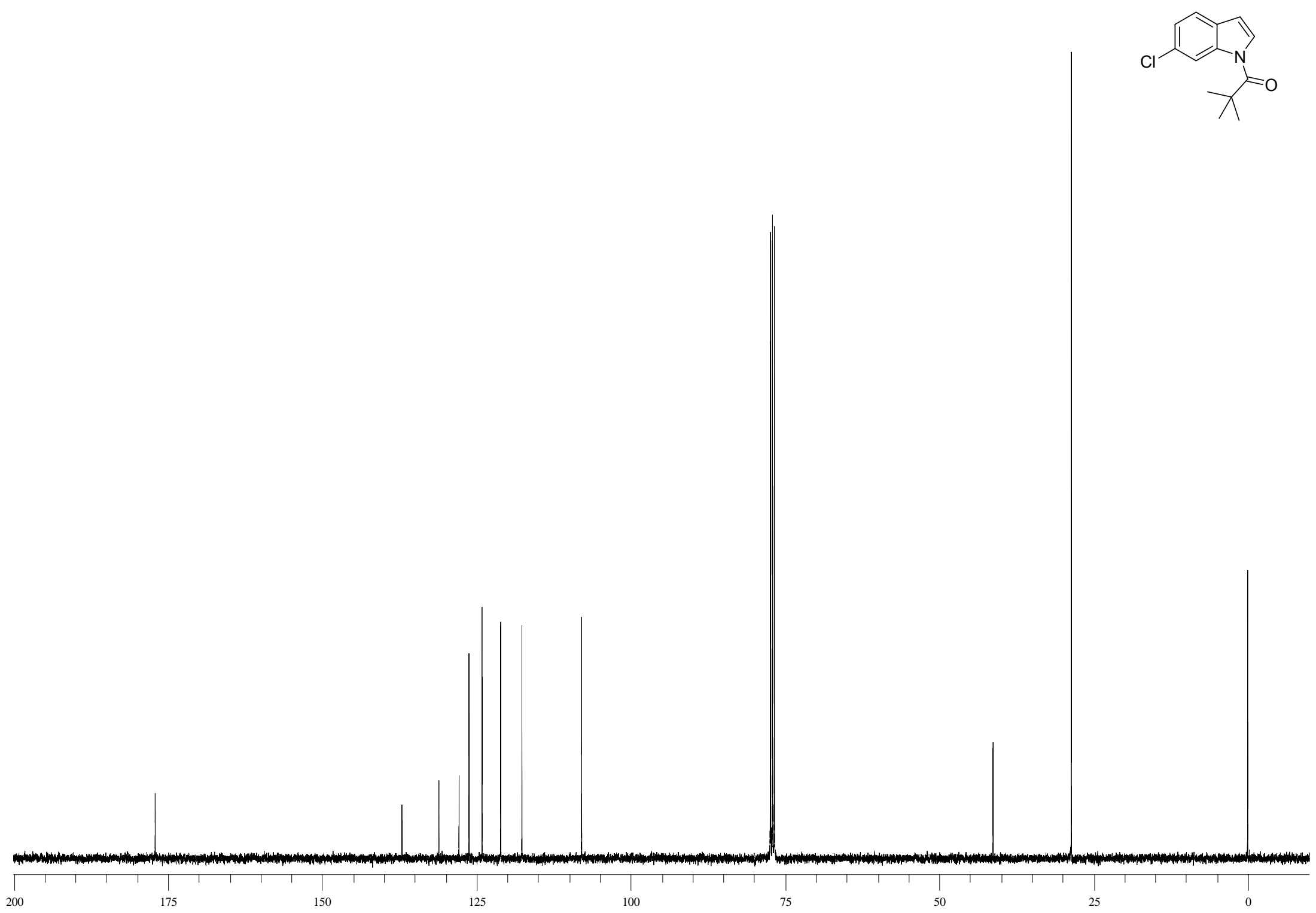




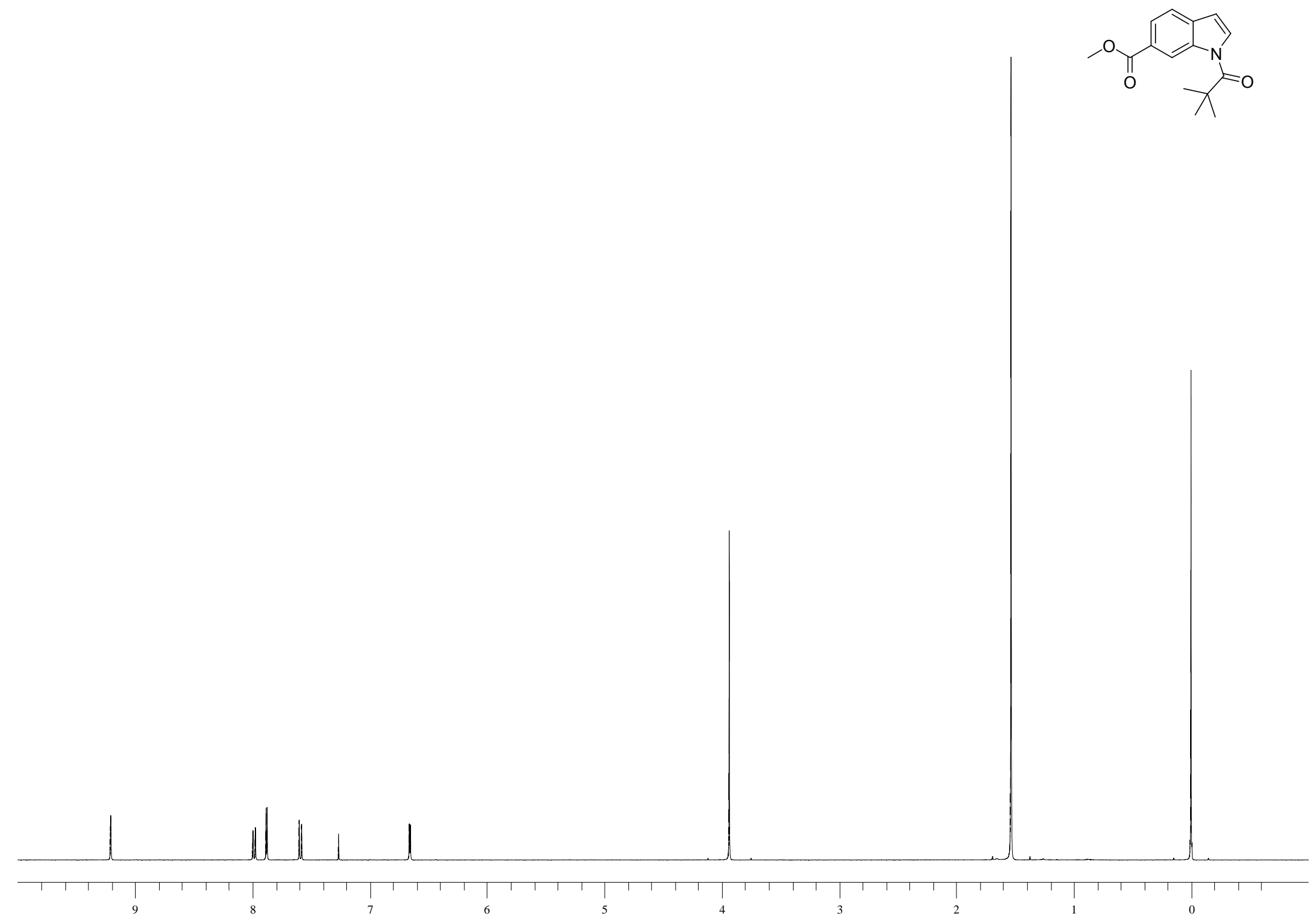




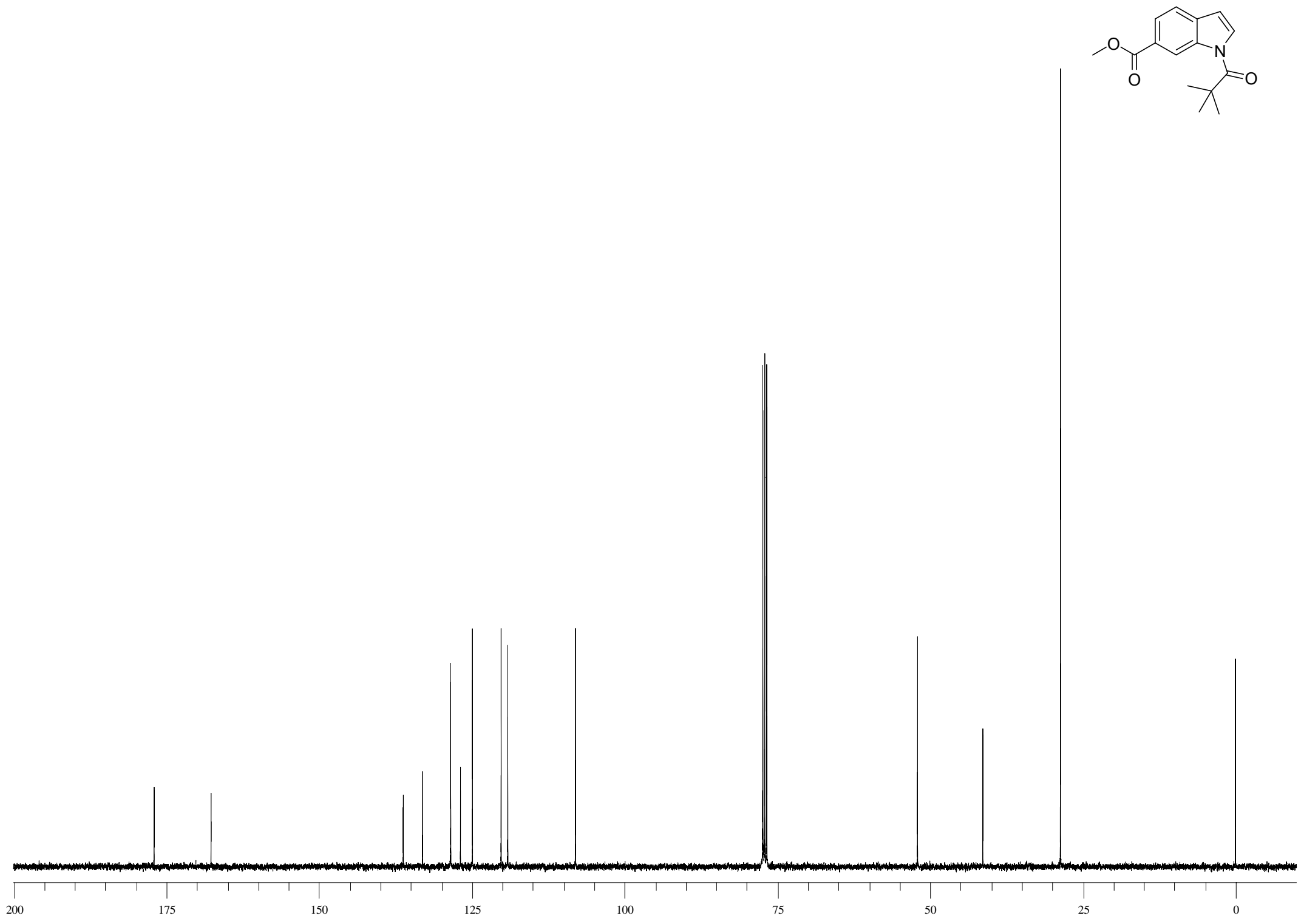



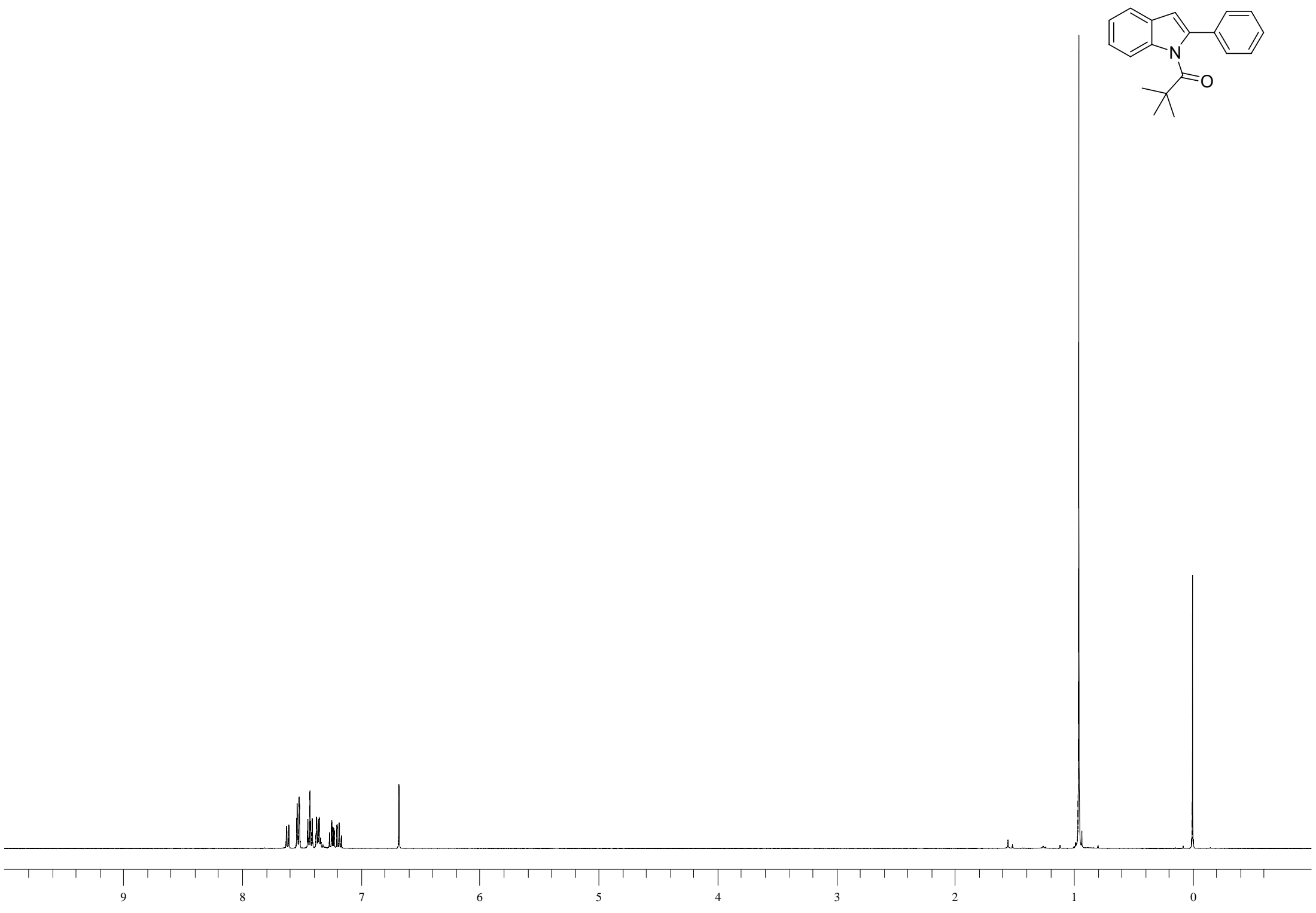


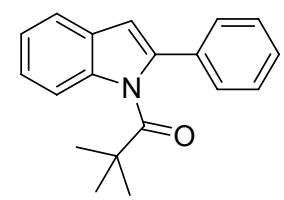

WH.

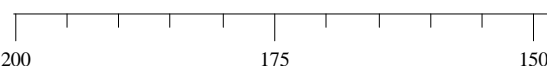




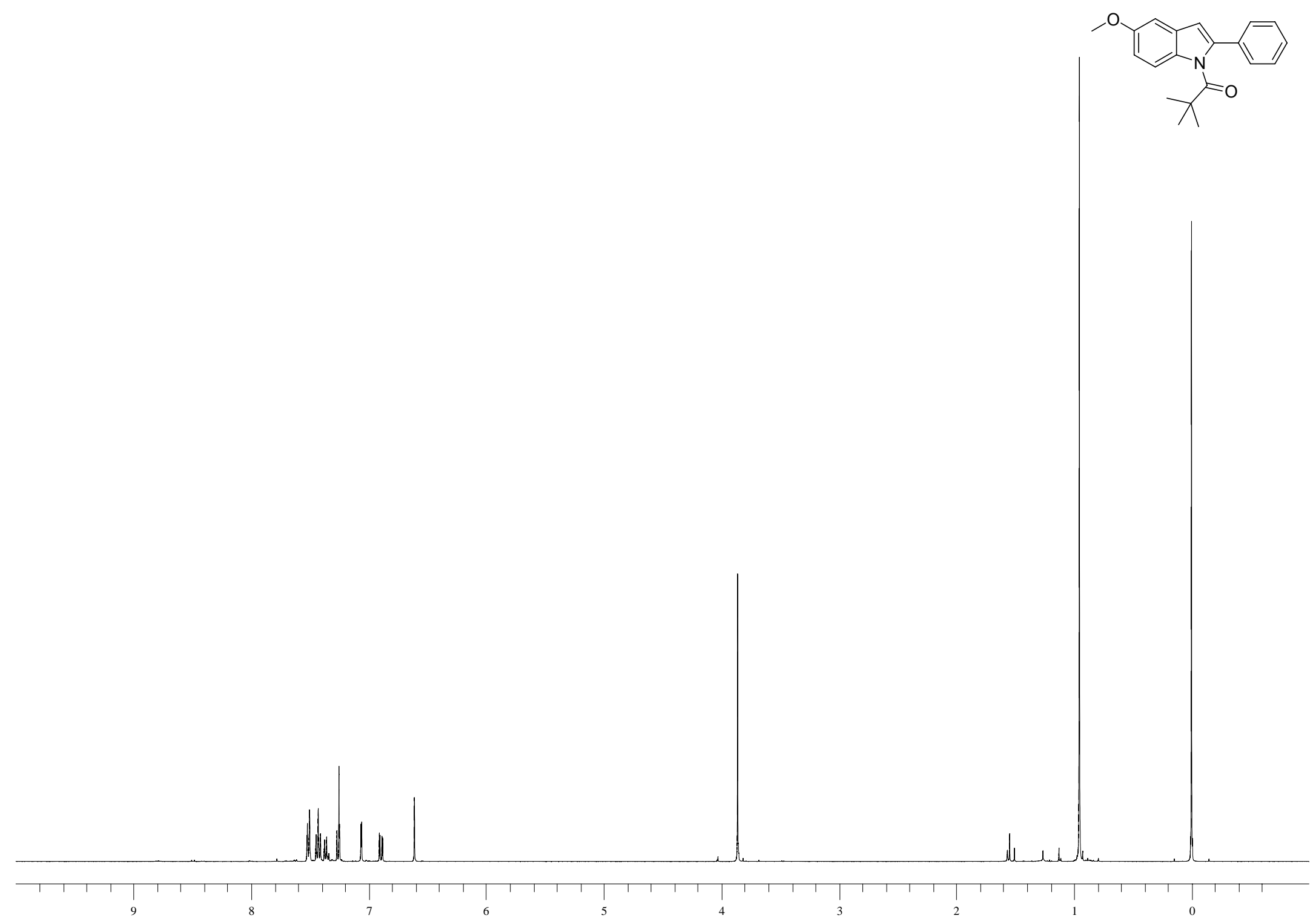




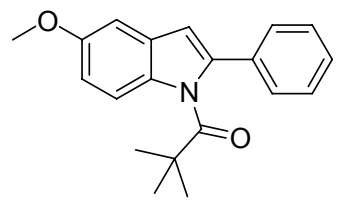

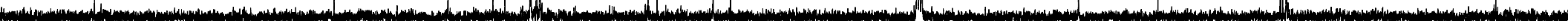

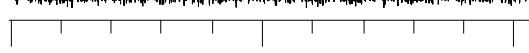




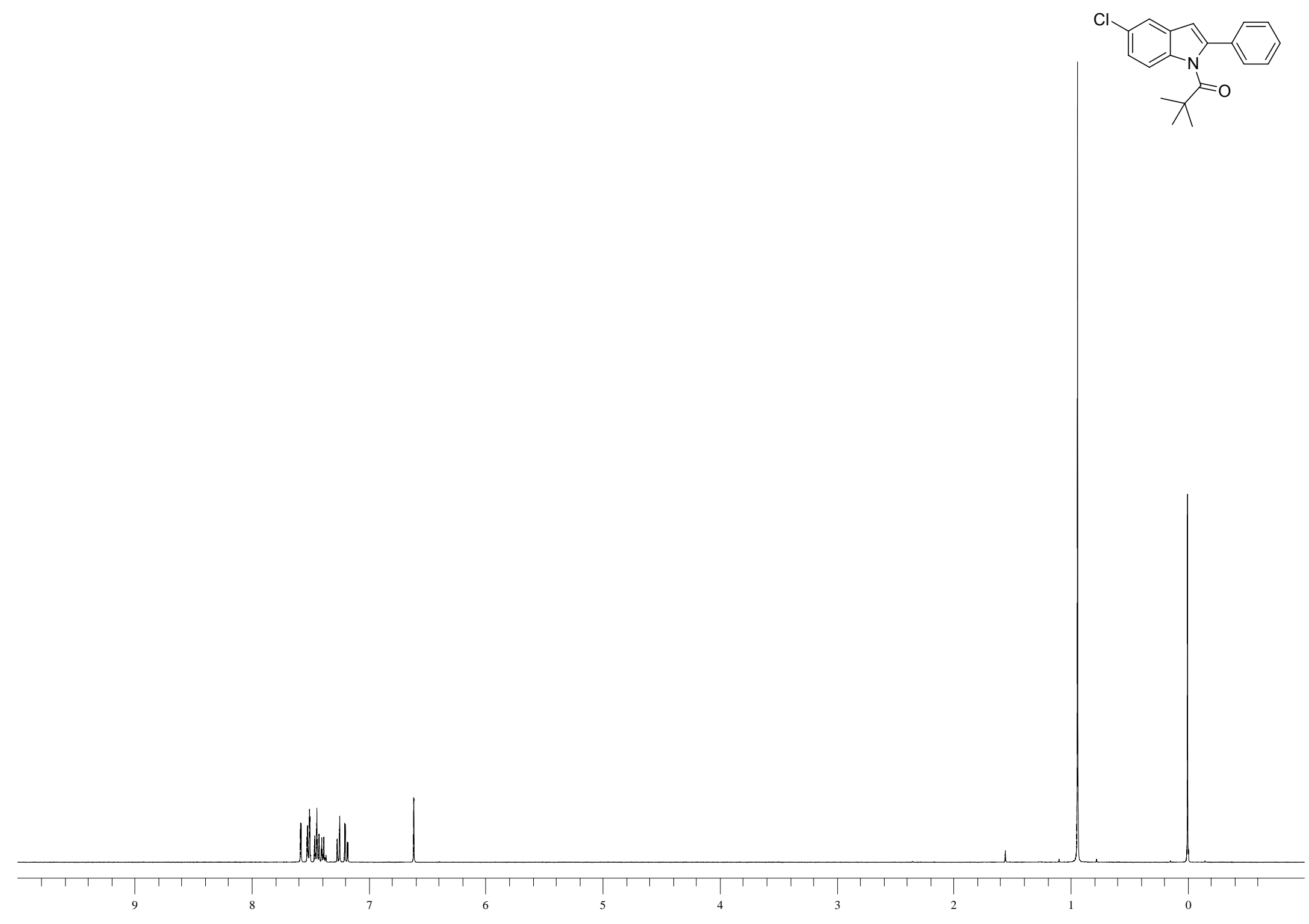



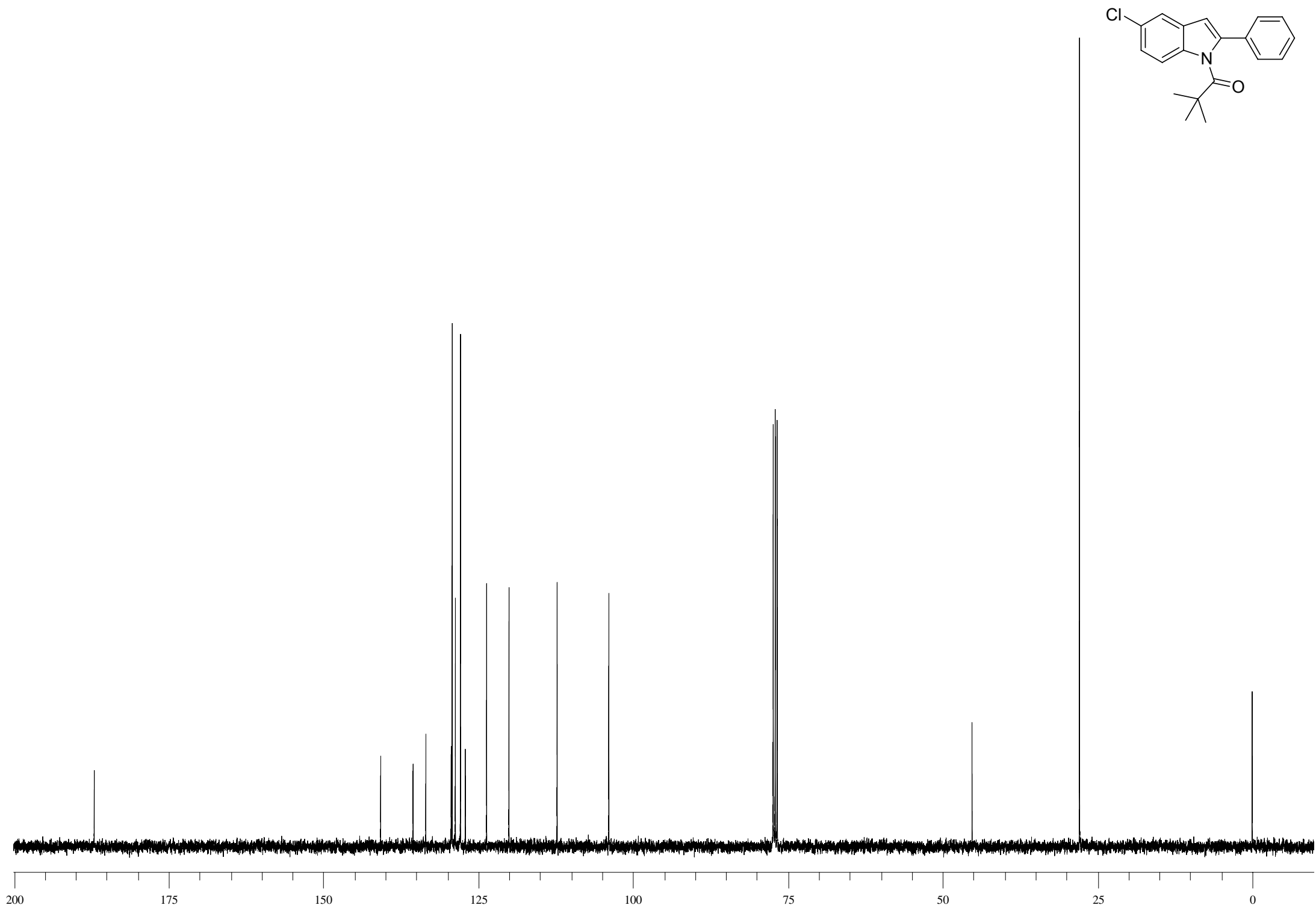


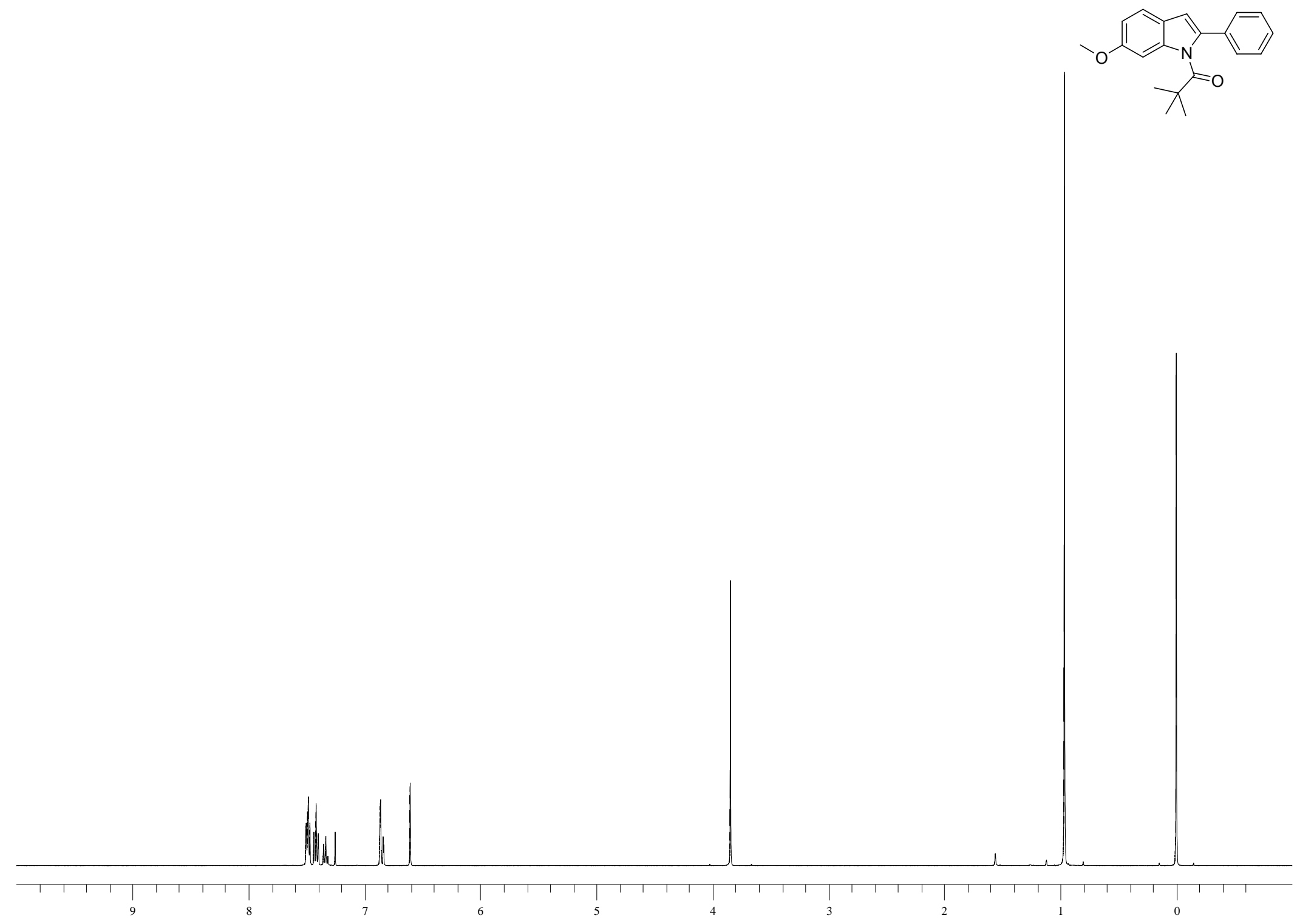




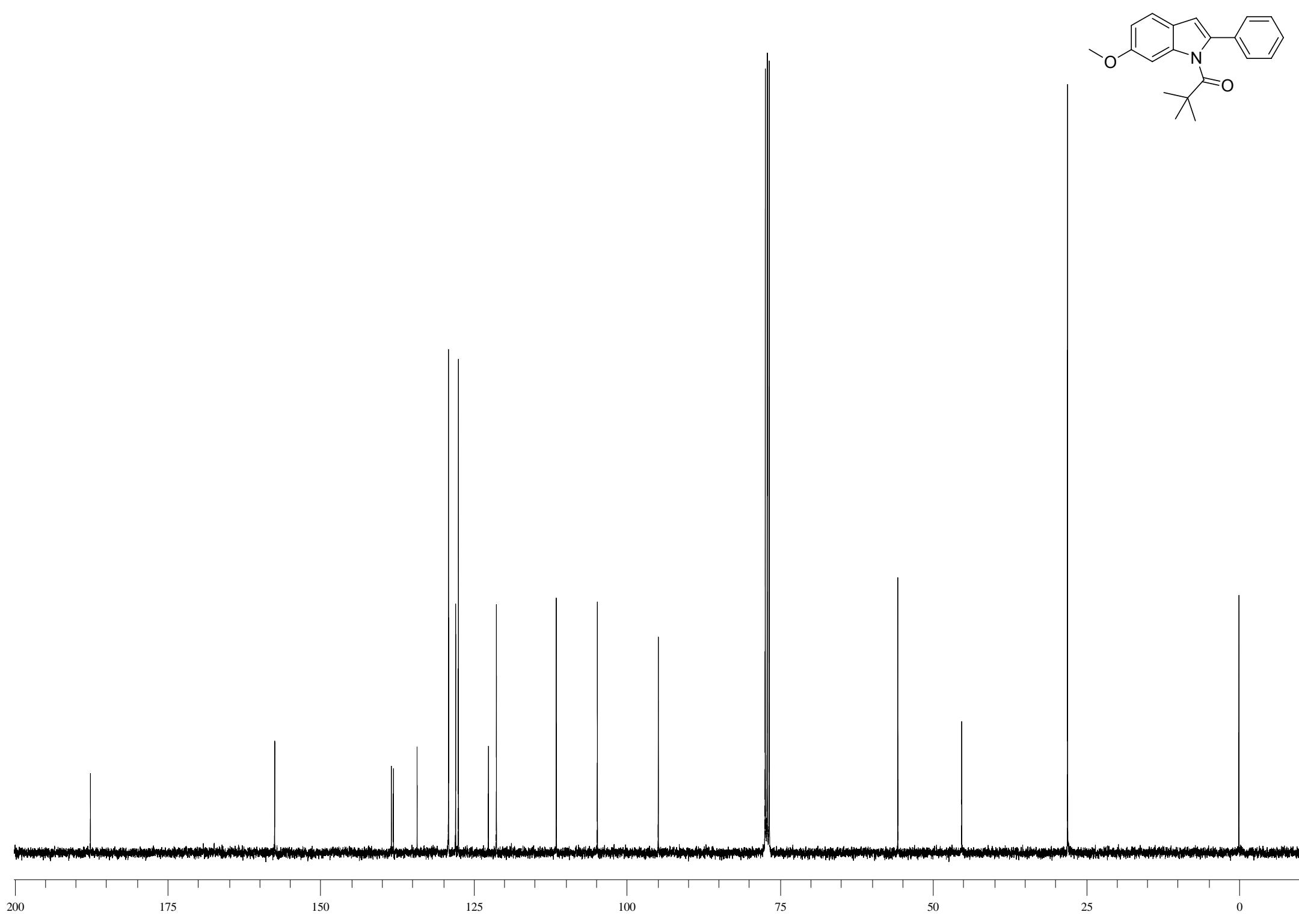




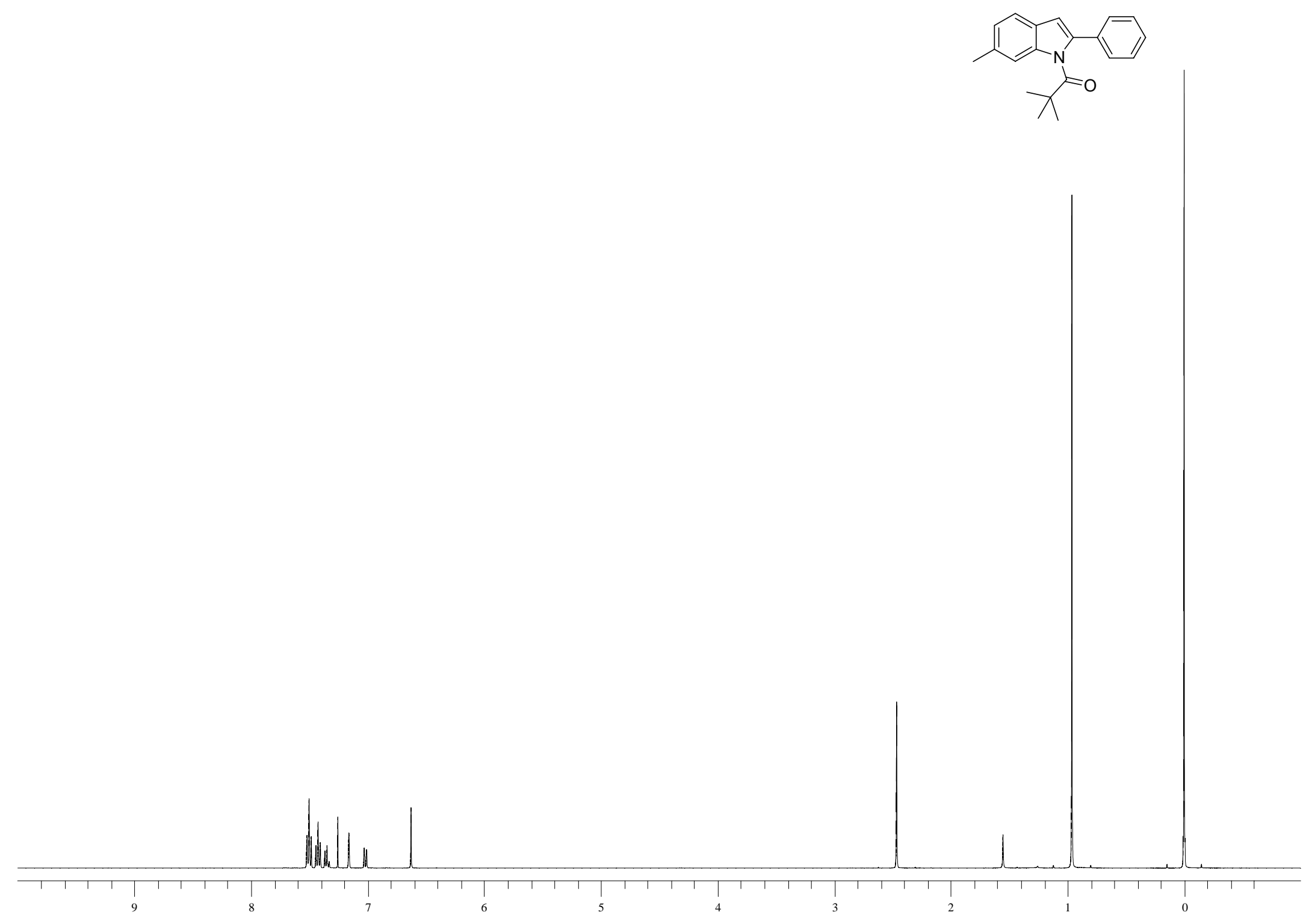




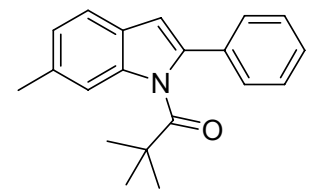

(1) 


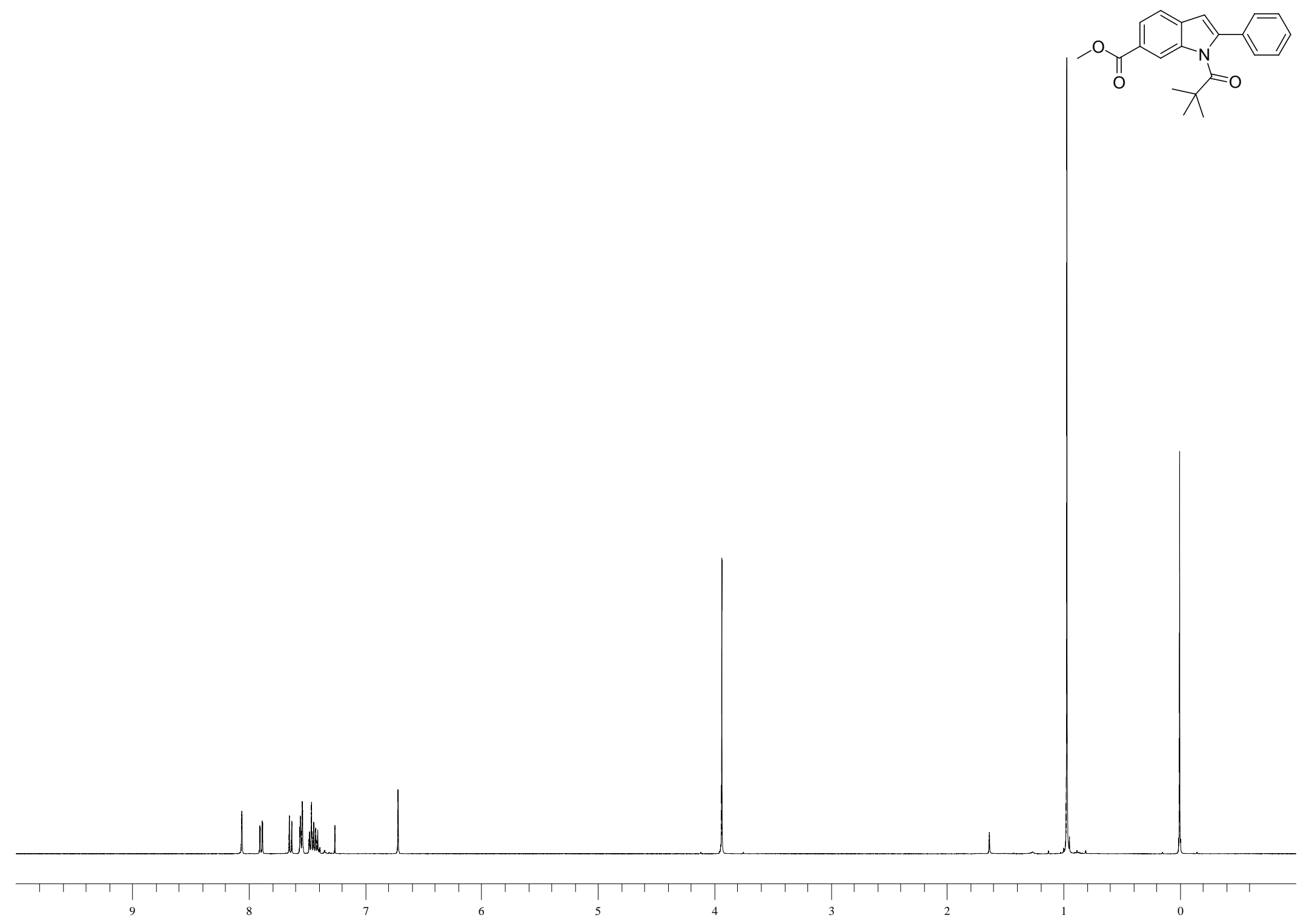




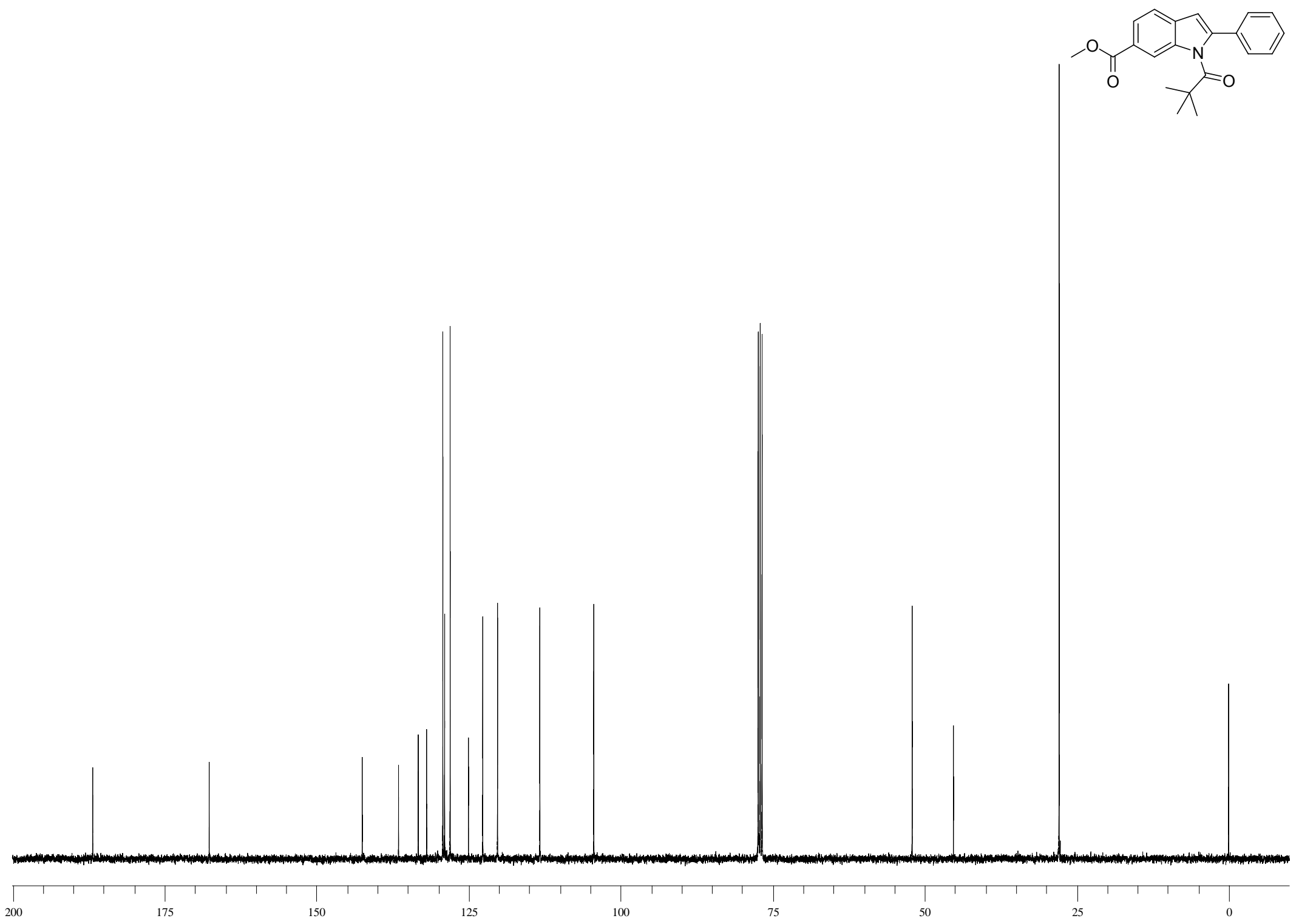




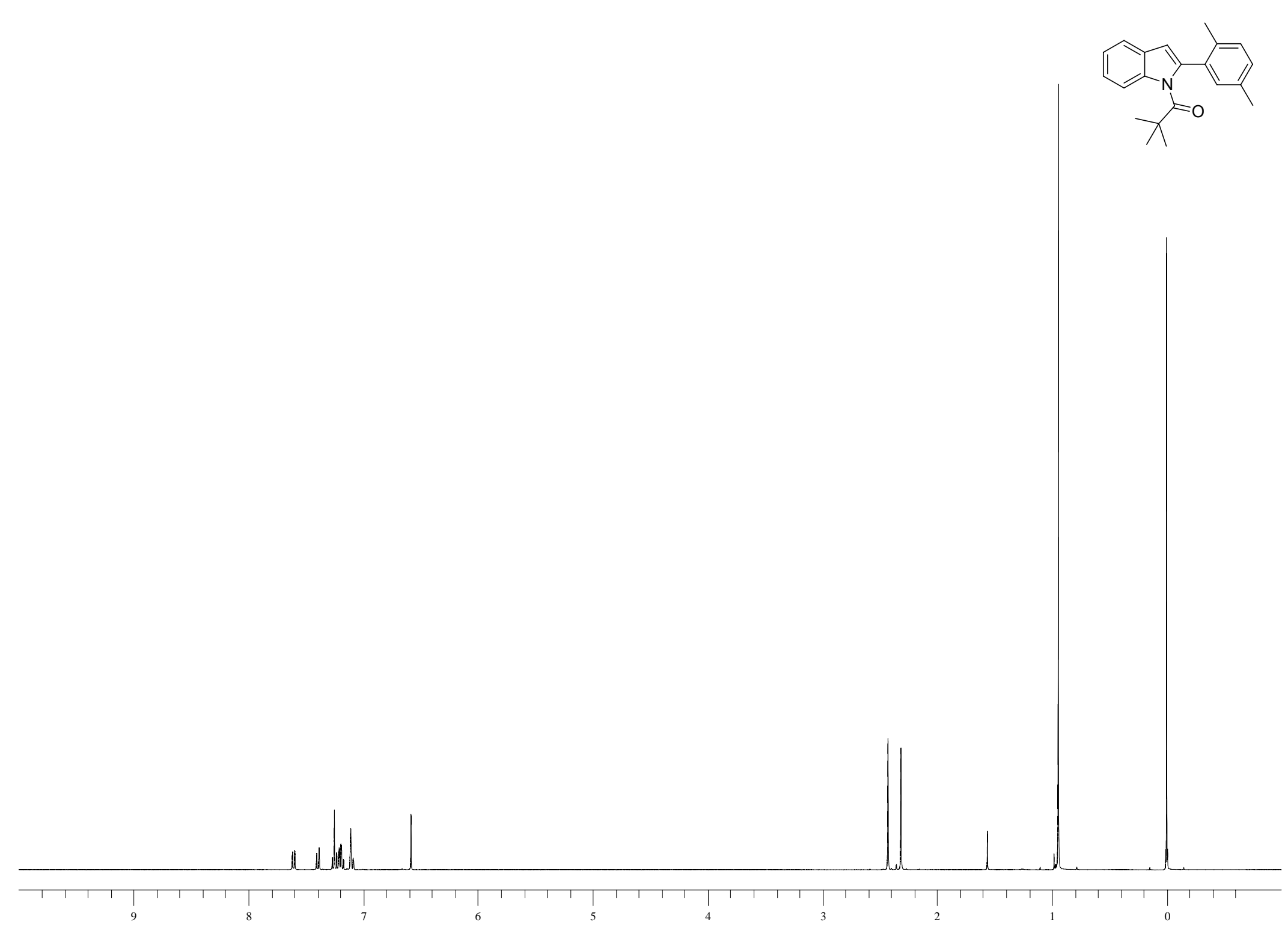




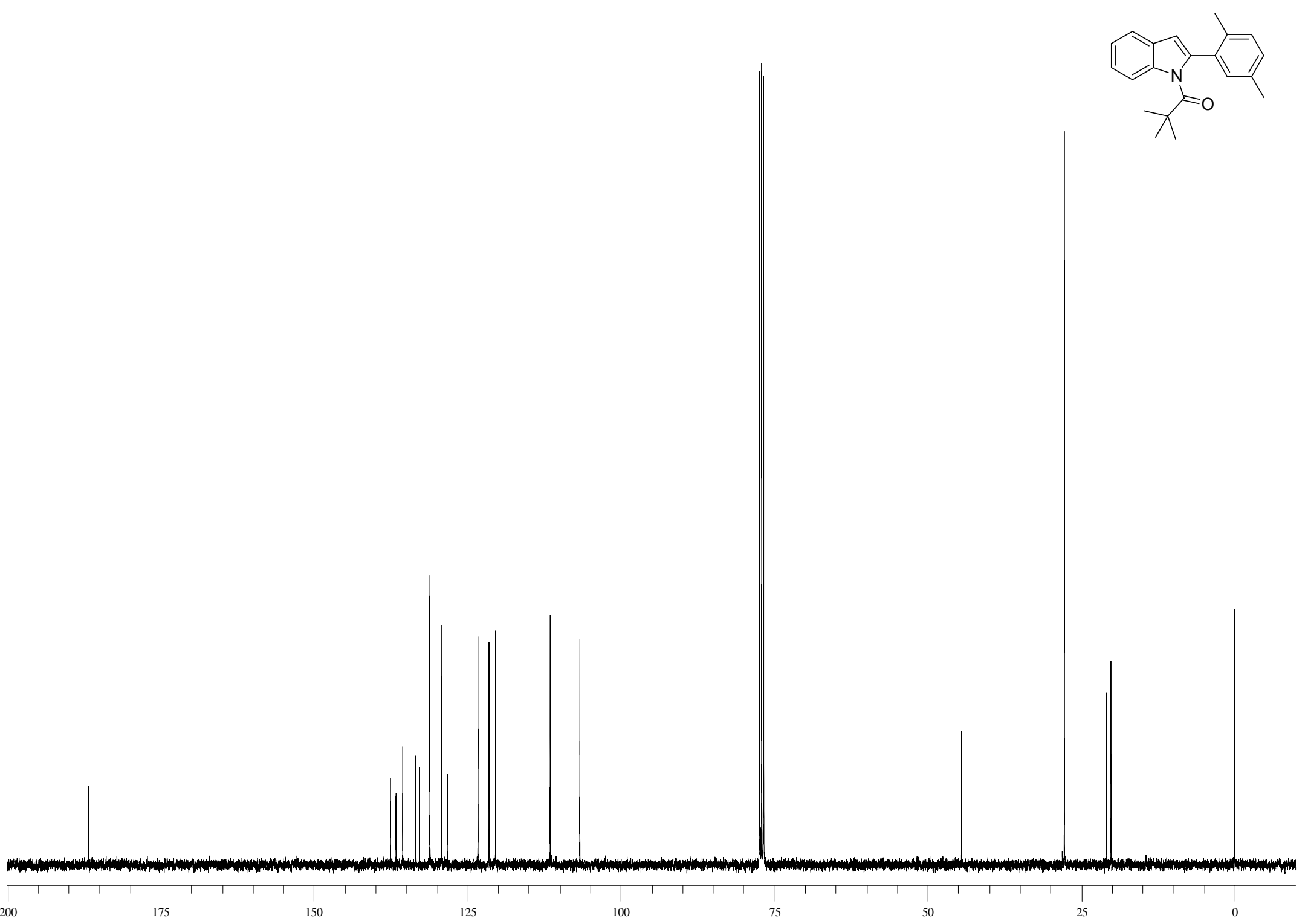




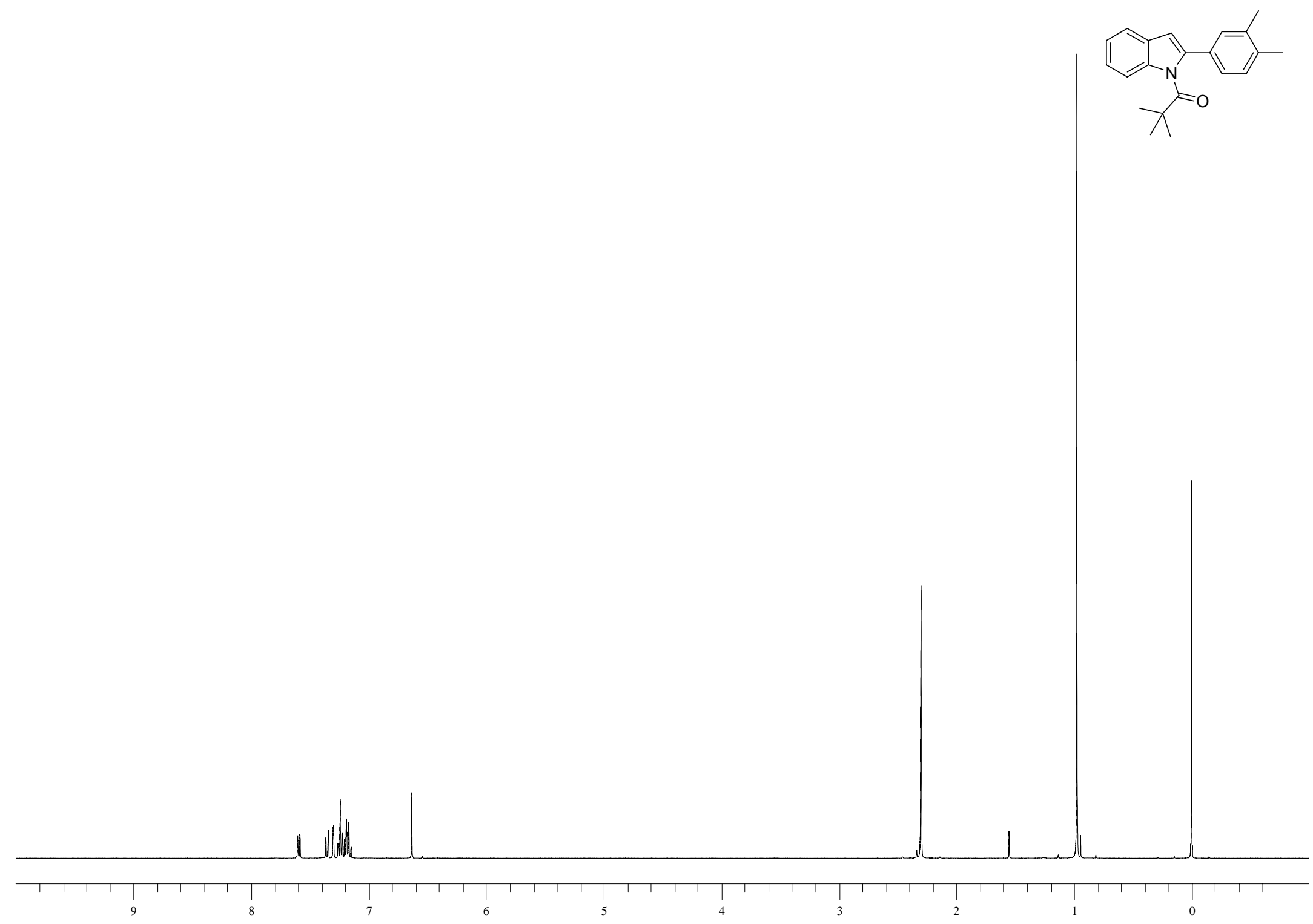




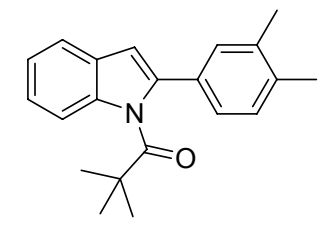

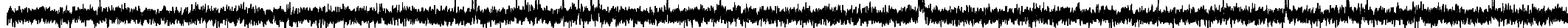




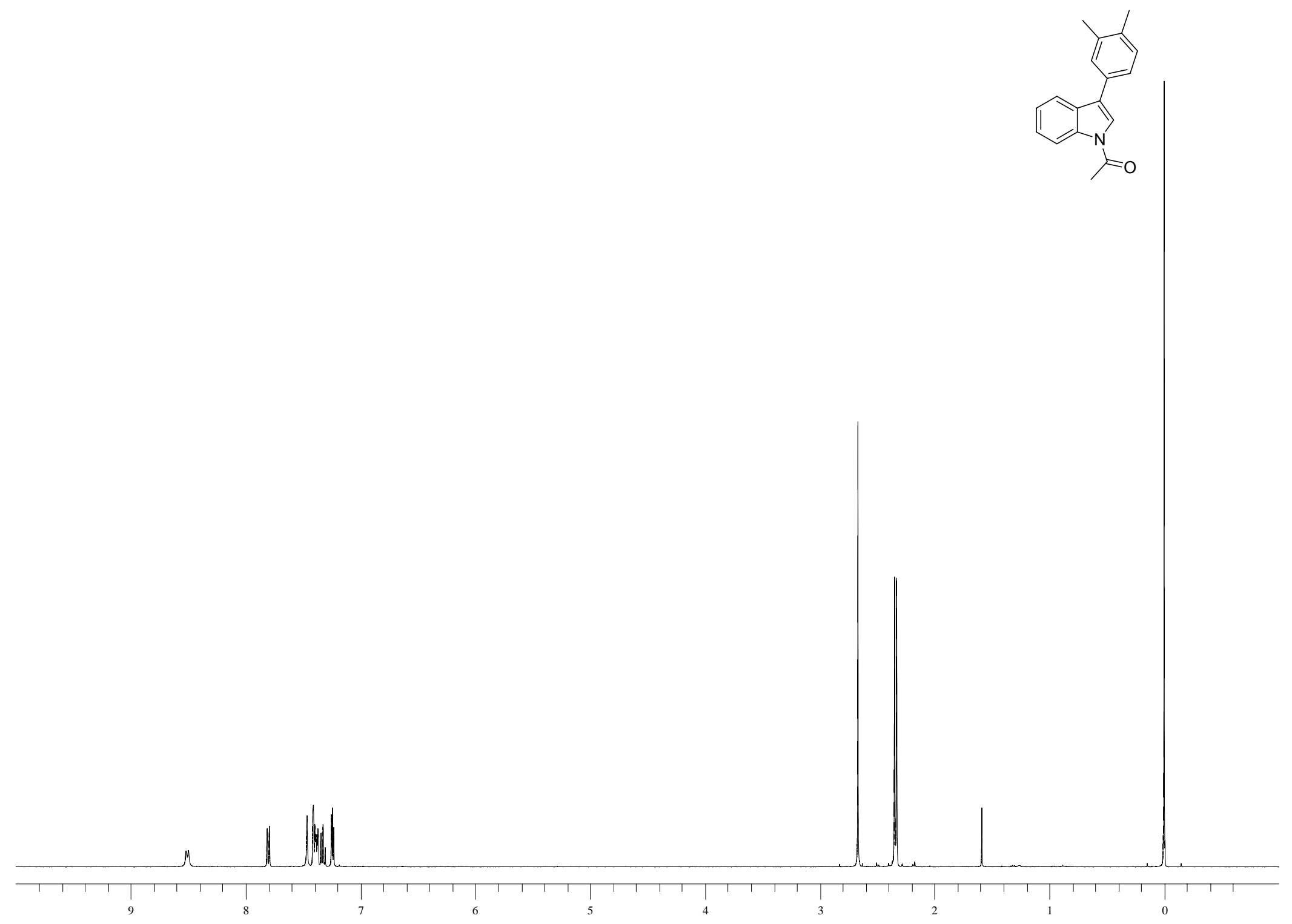




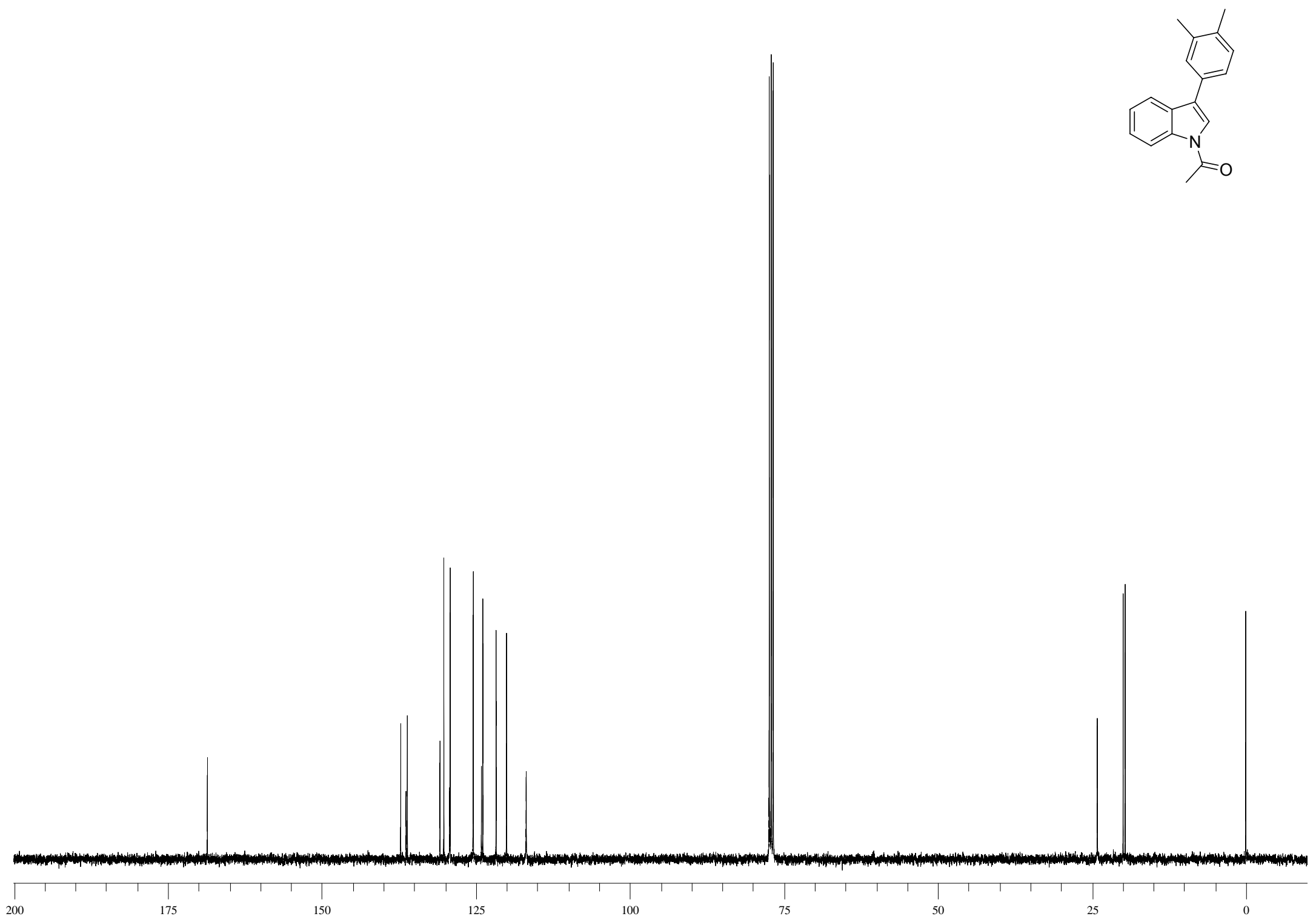




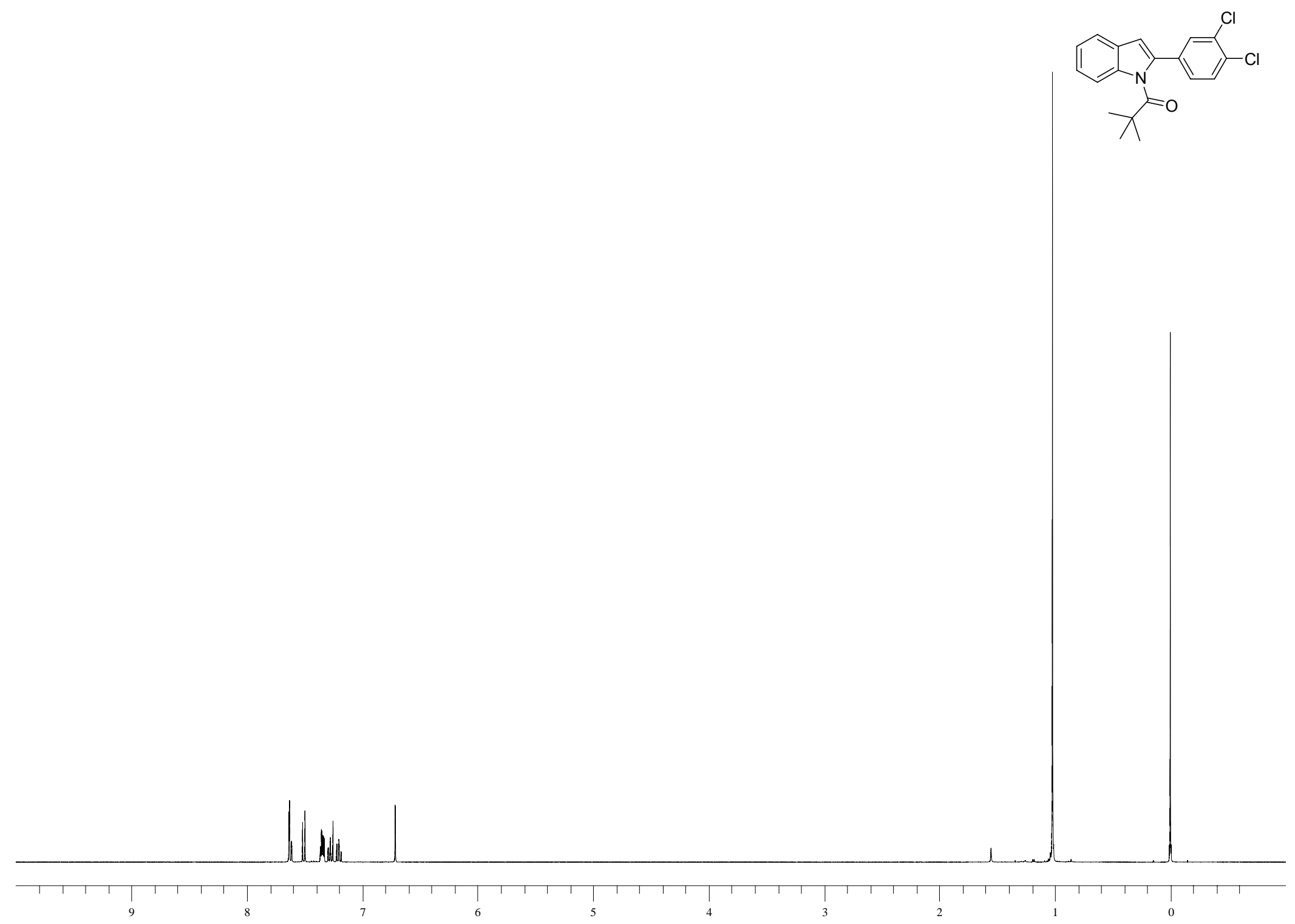




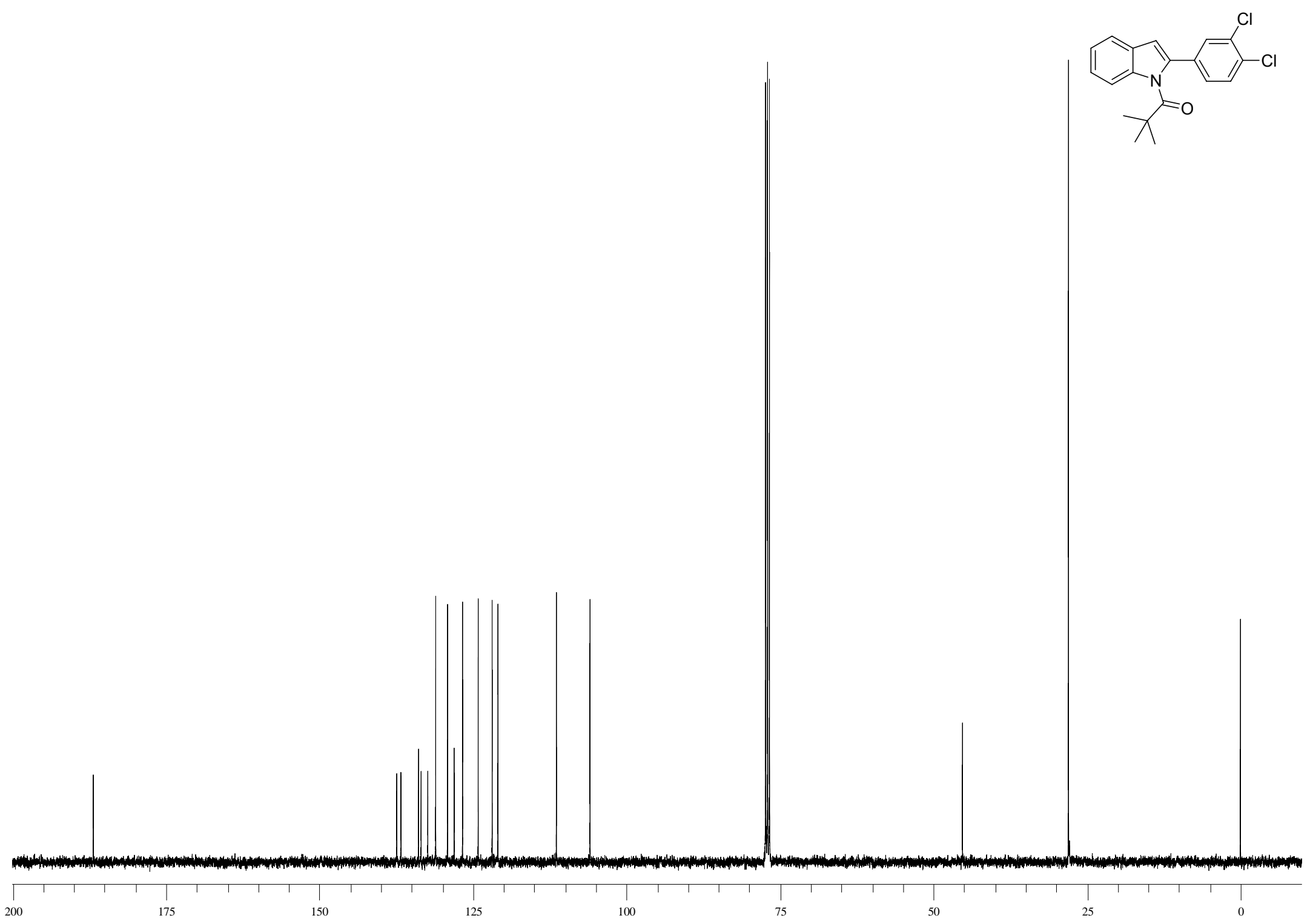



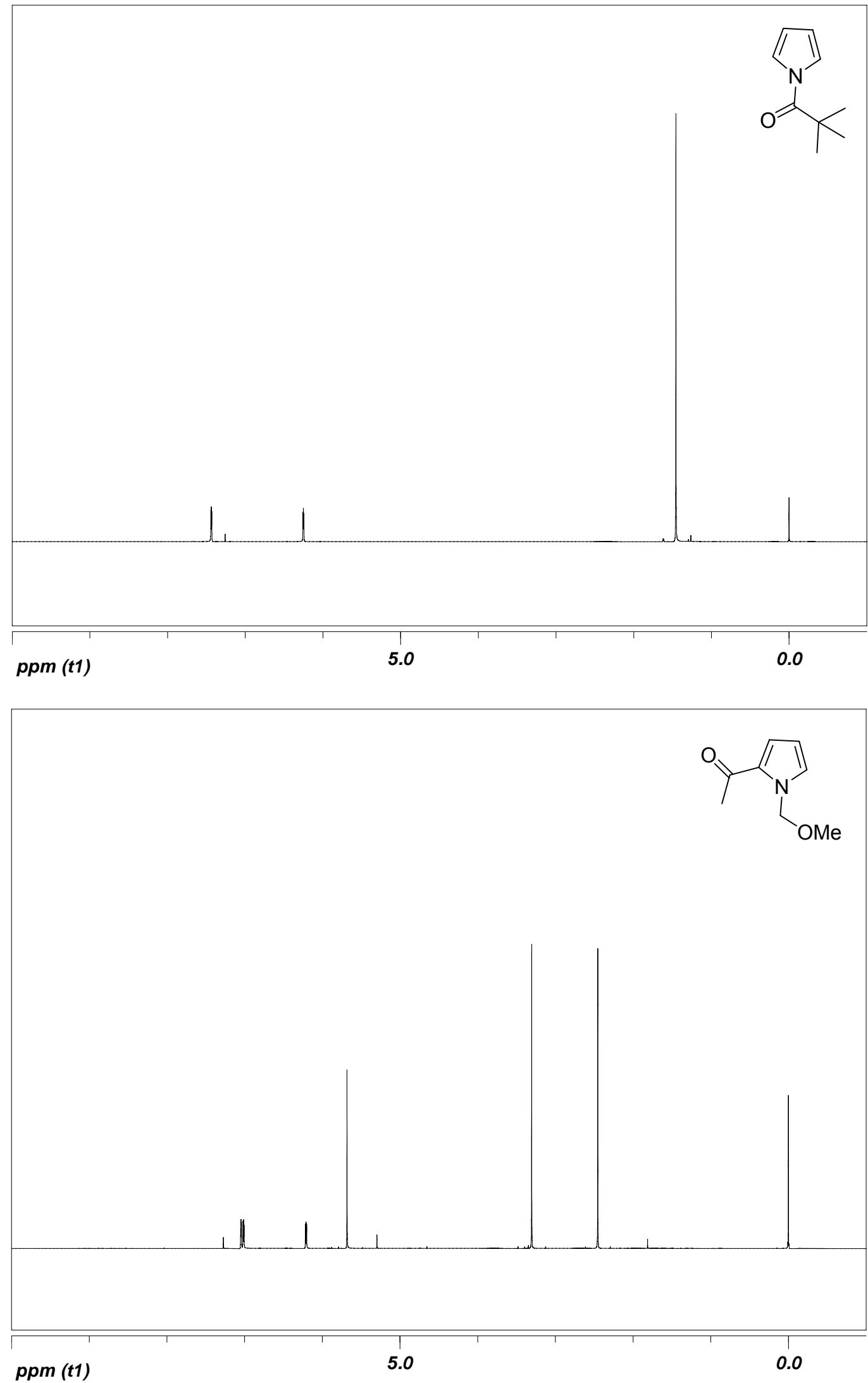


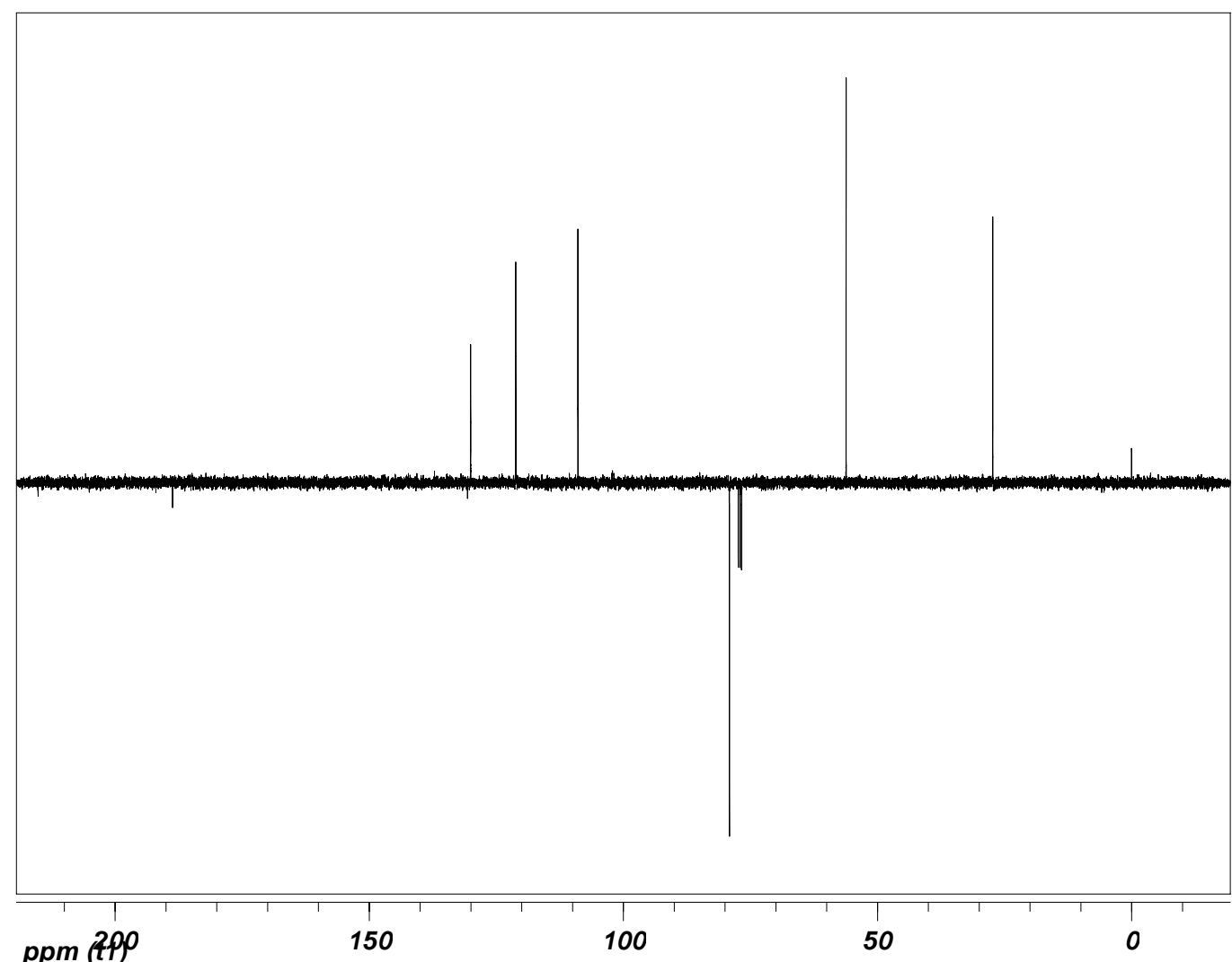

ppm (29)

150

100

50
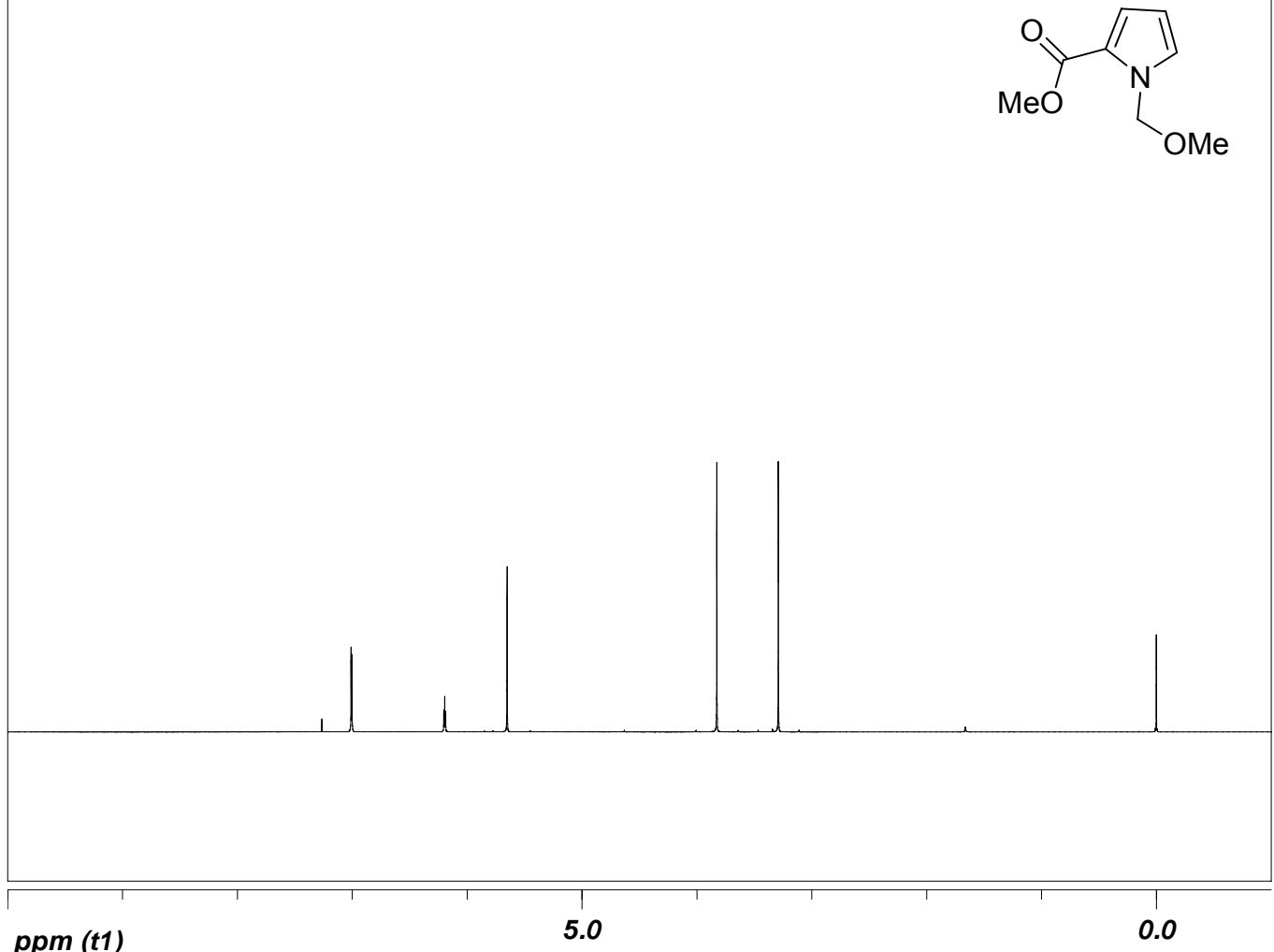

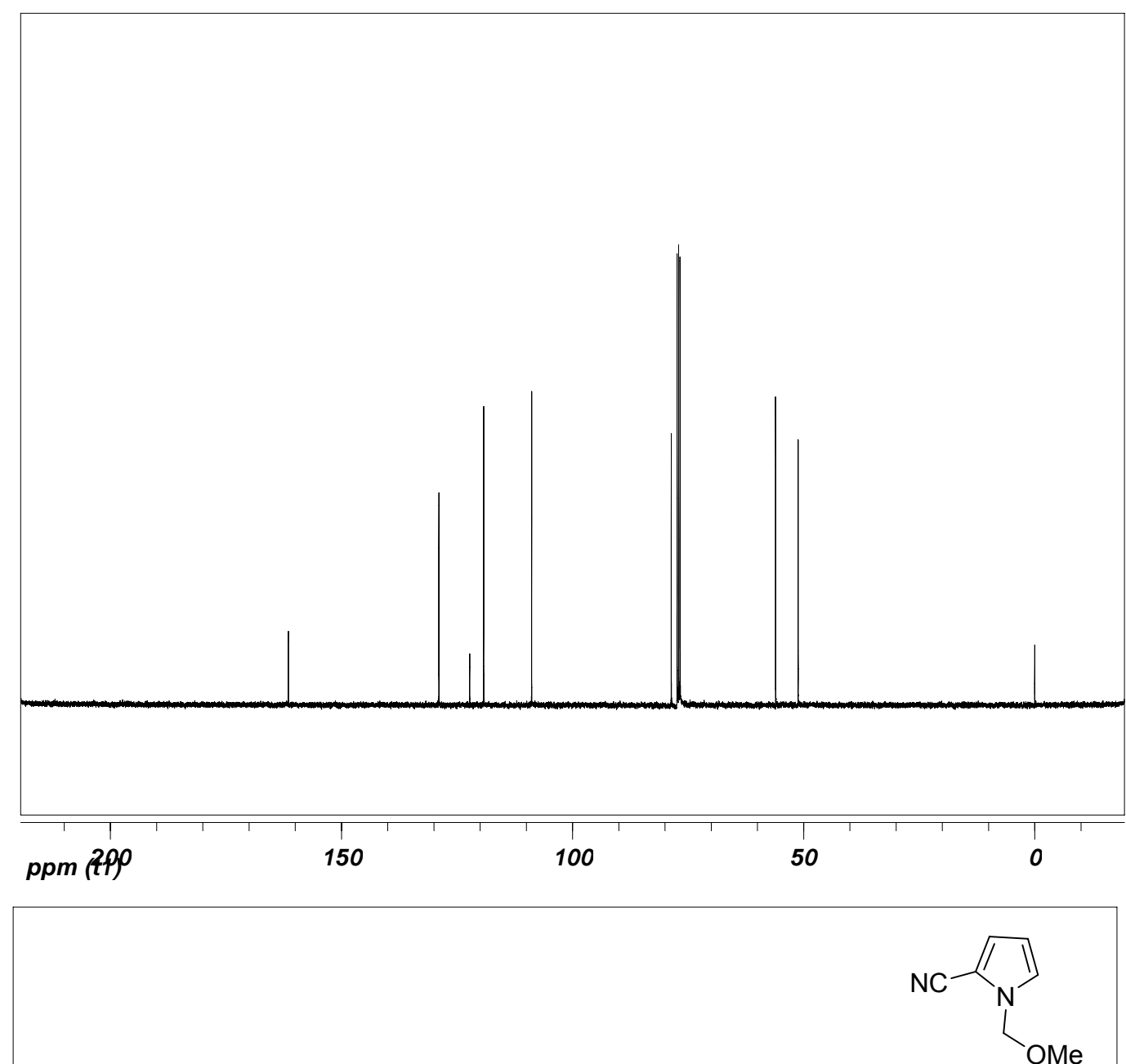

ppm (t1) 


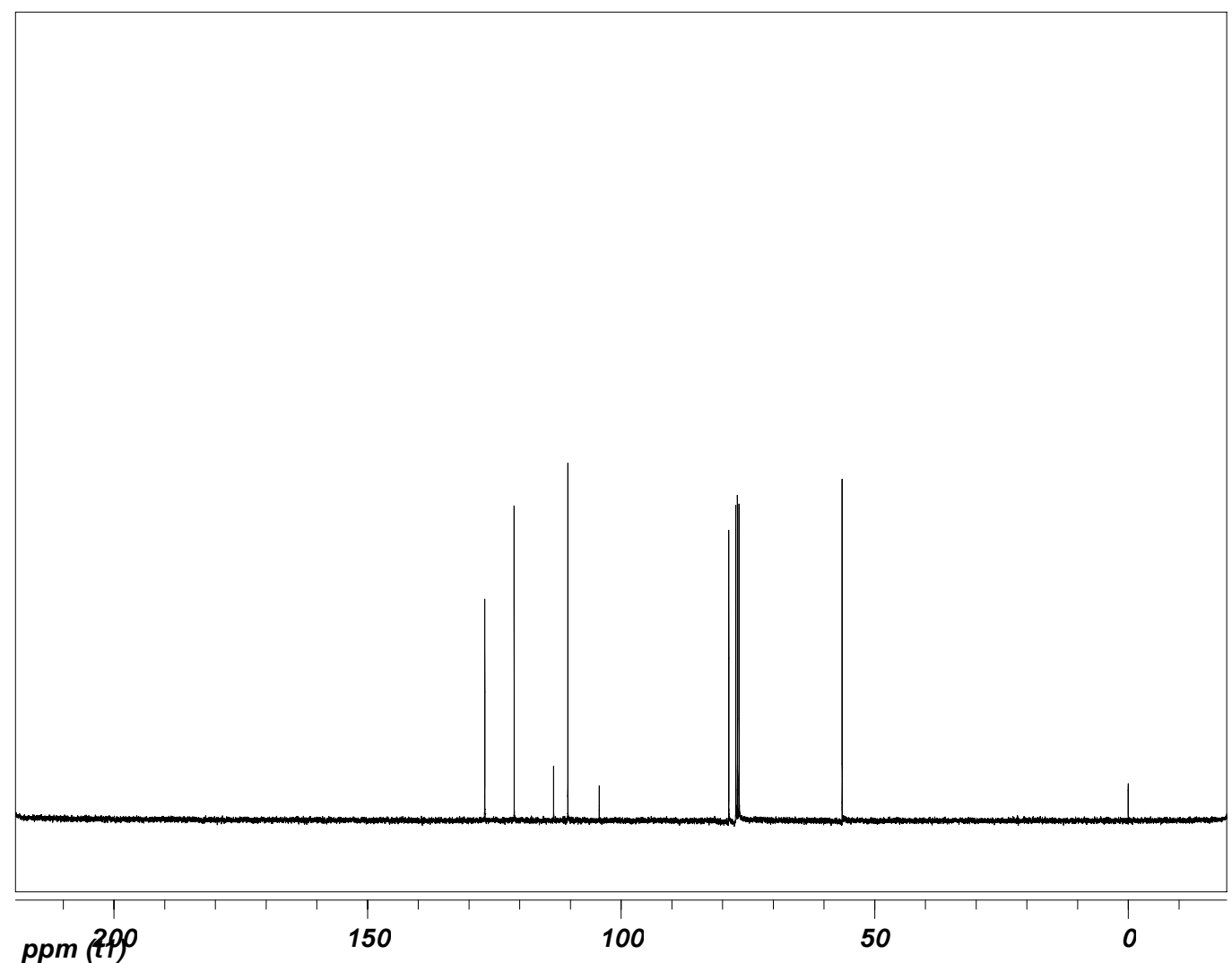

ppm (t2P)
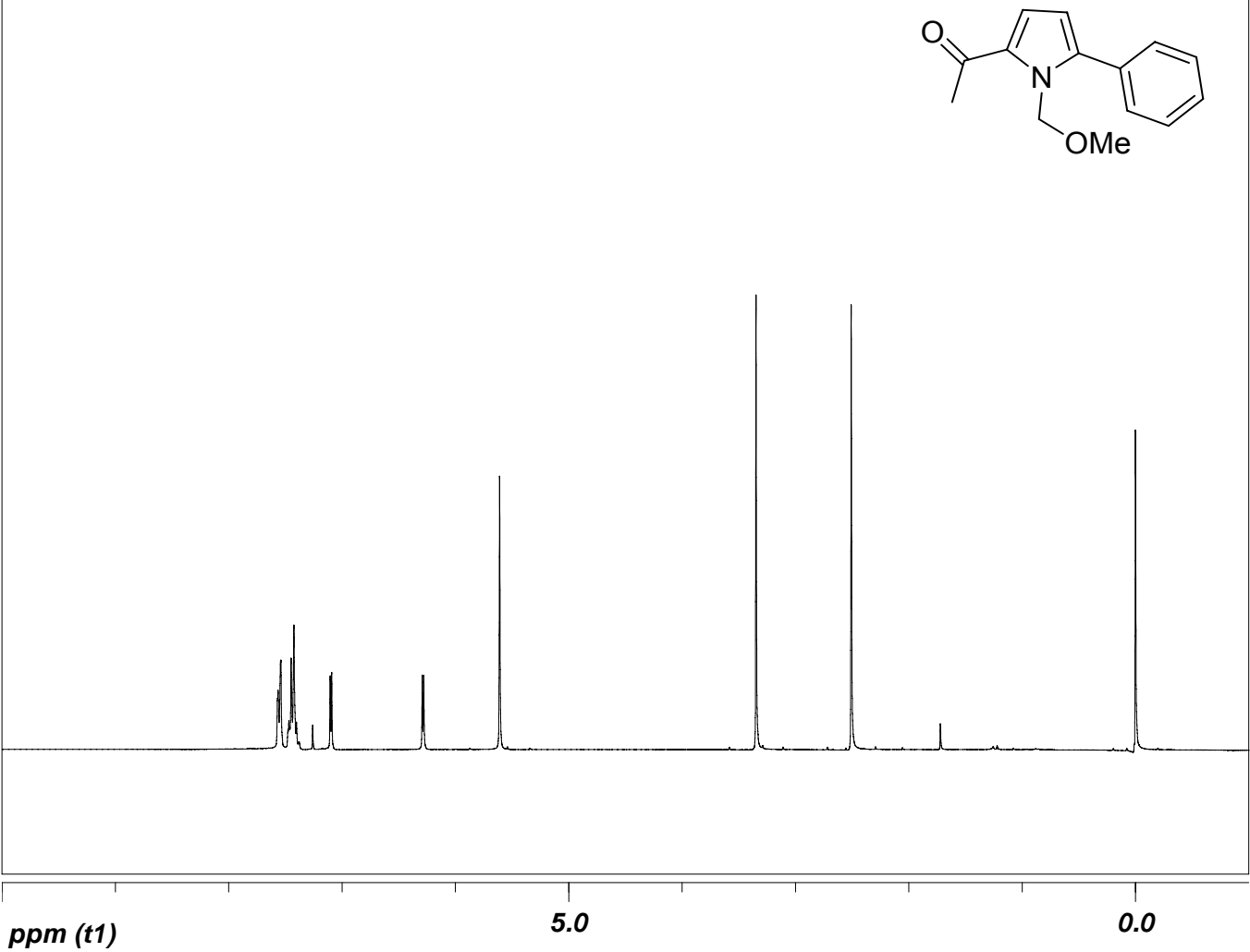

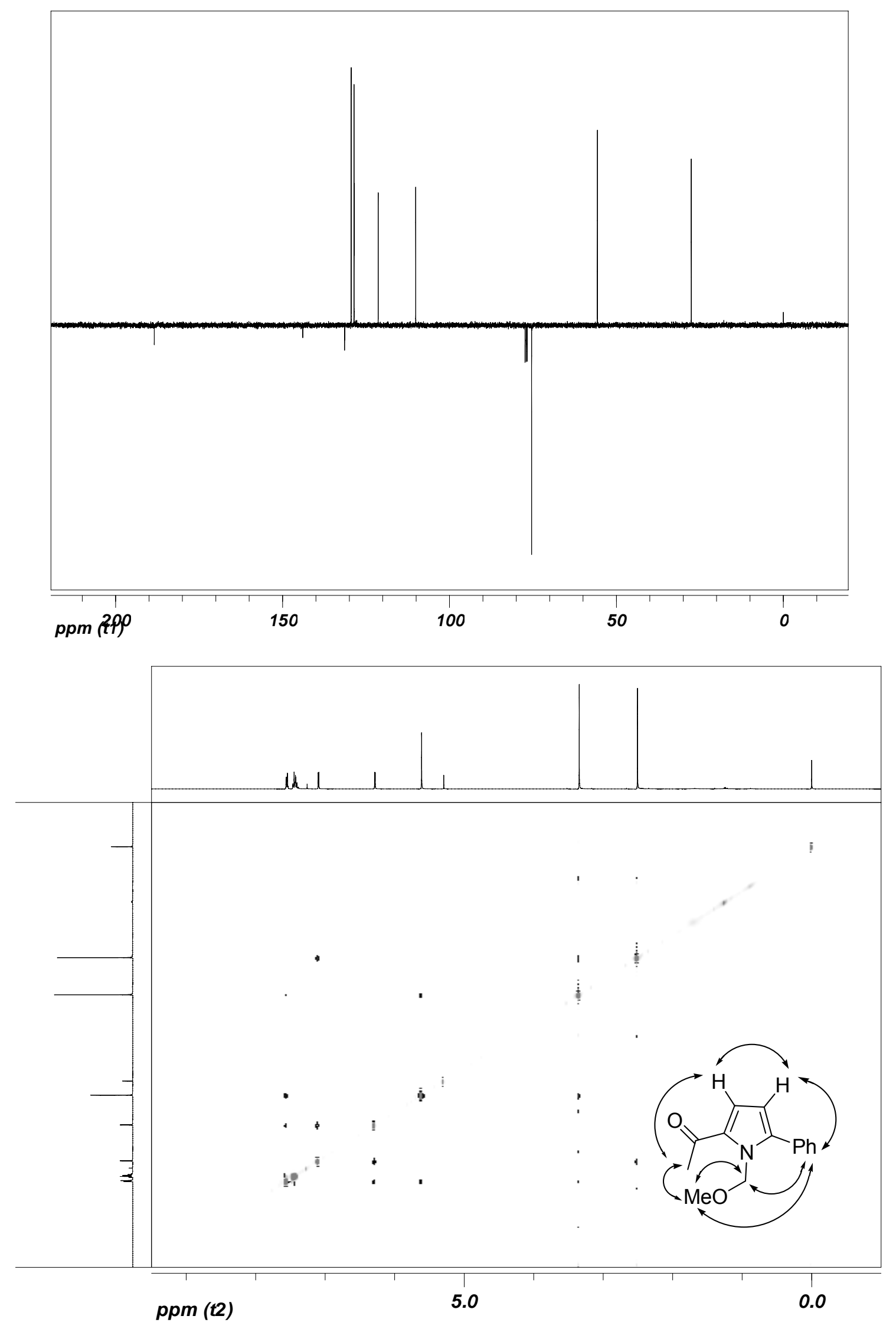


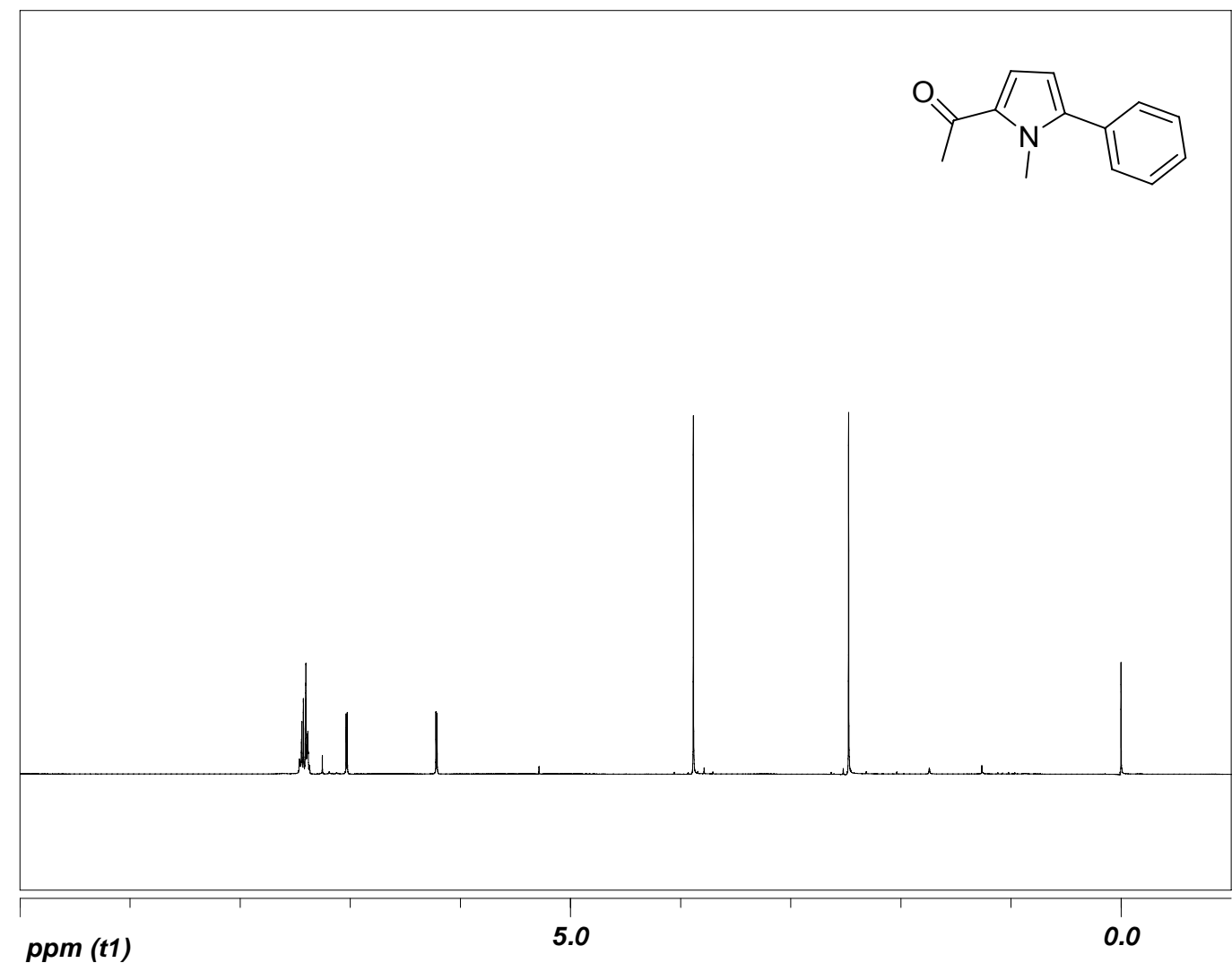

ppm (t1)

5.0

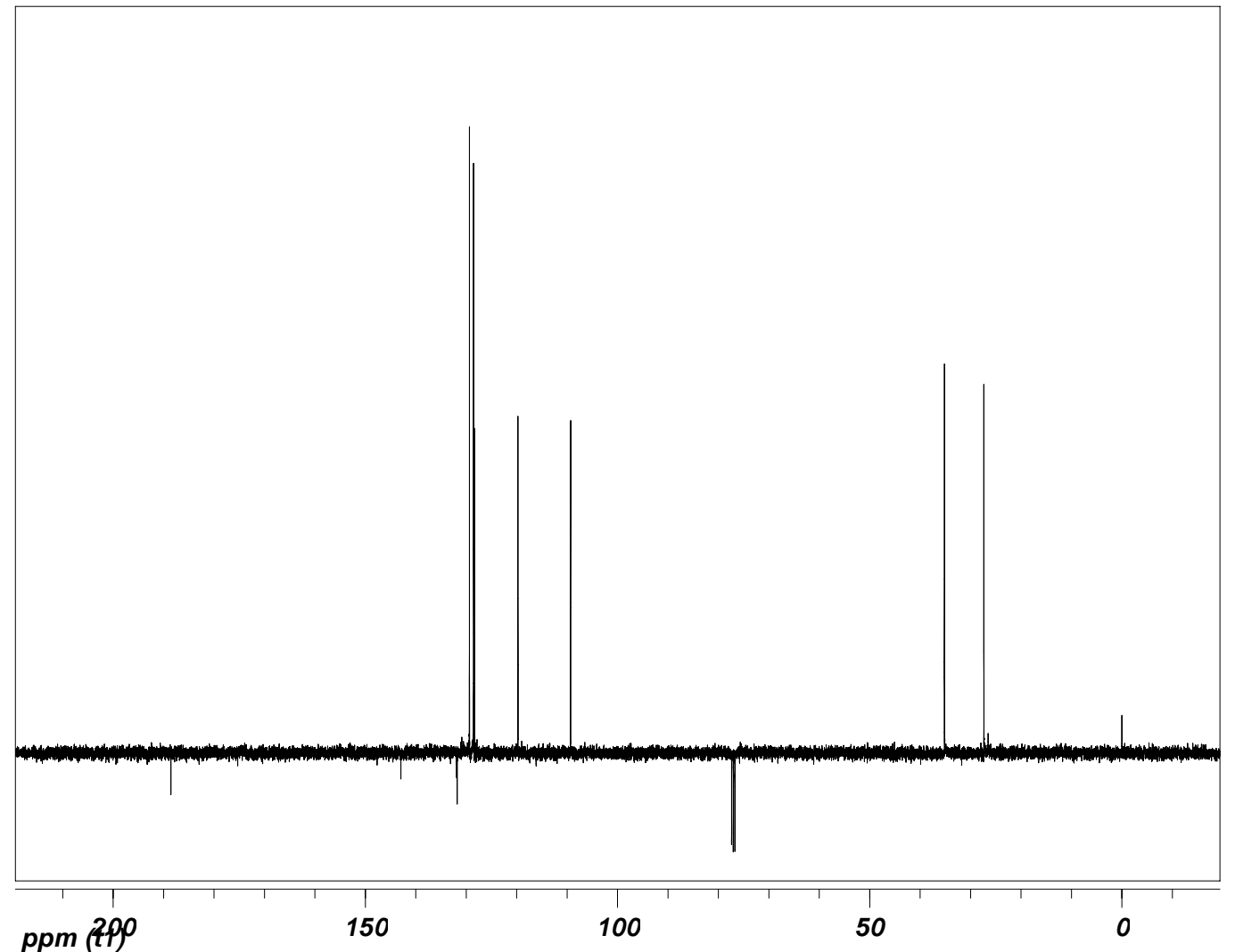



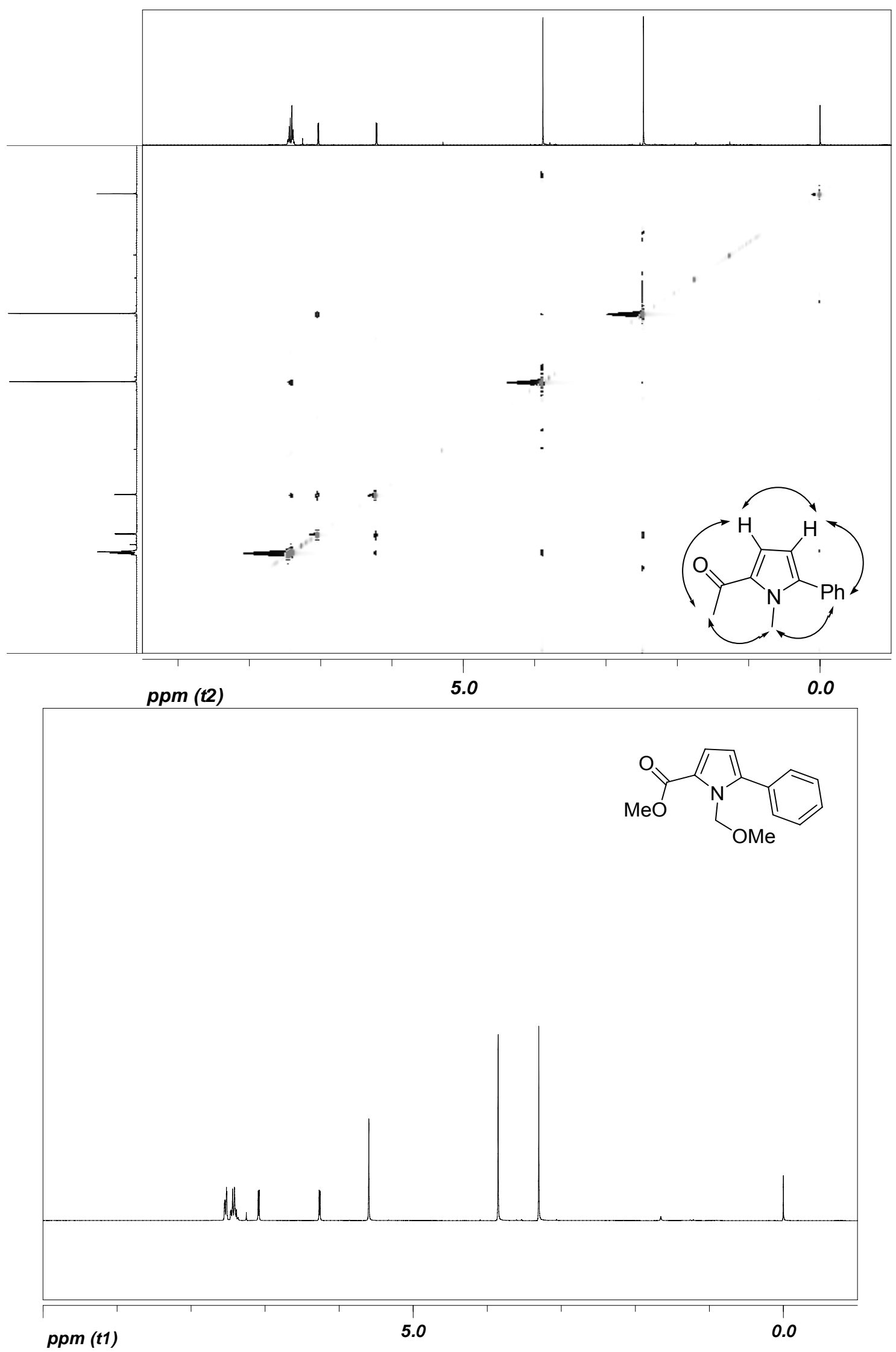


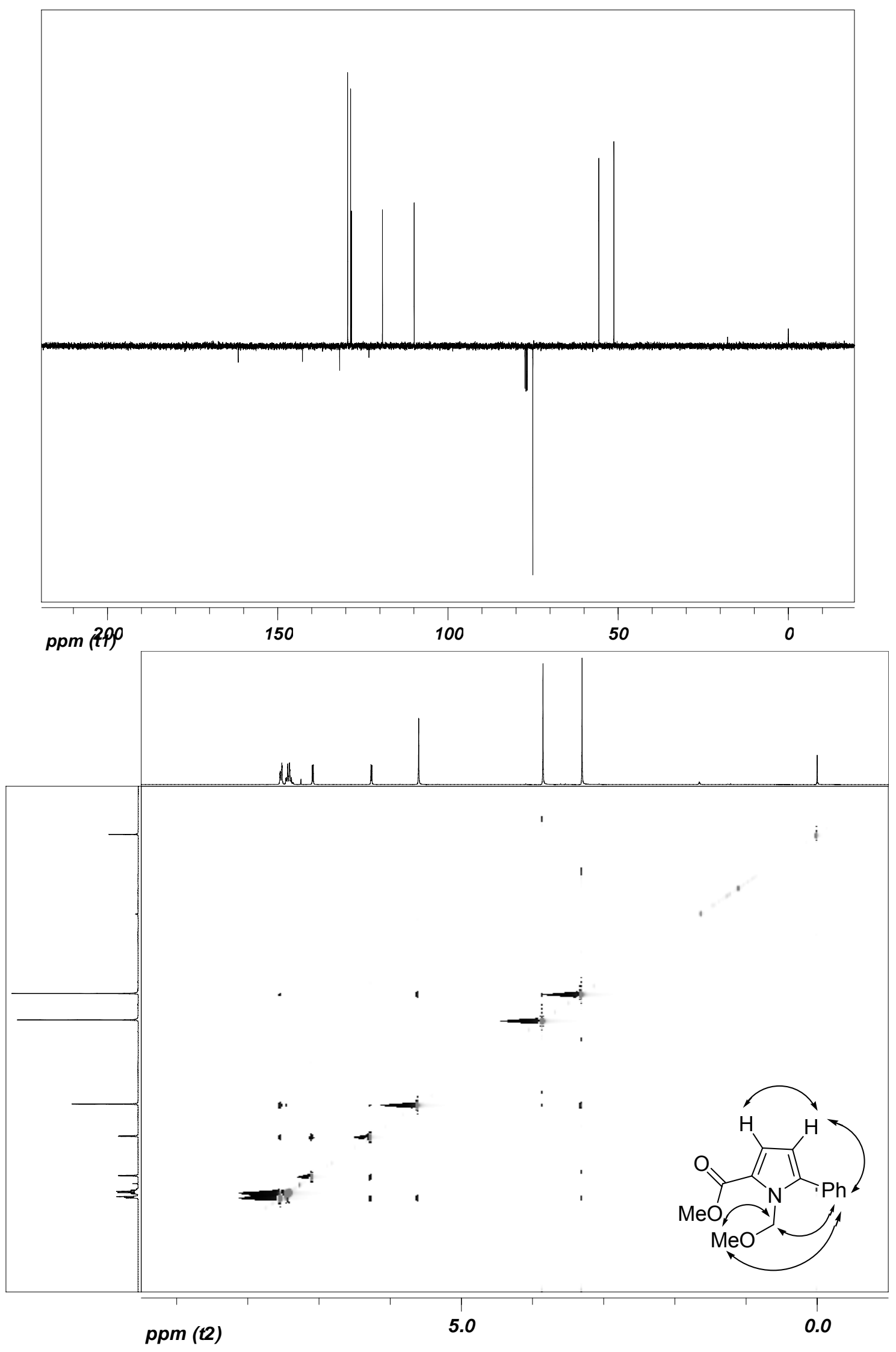




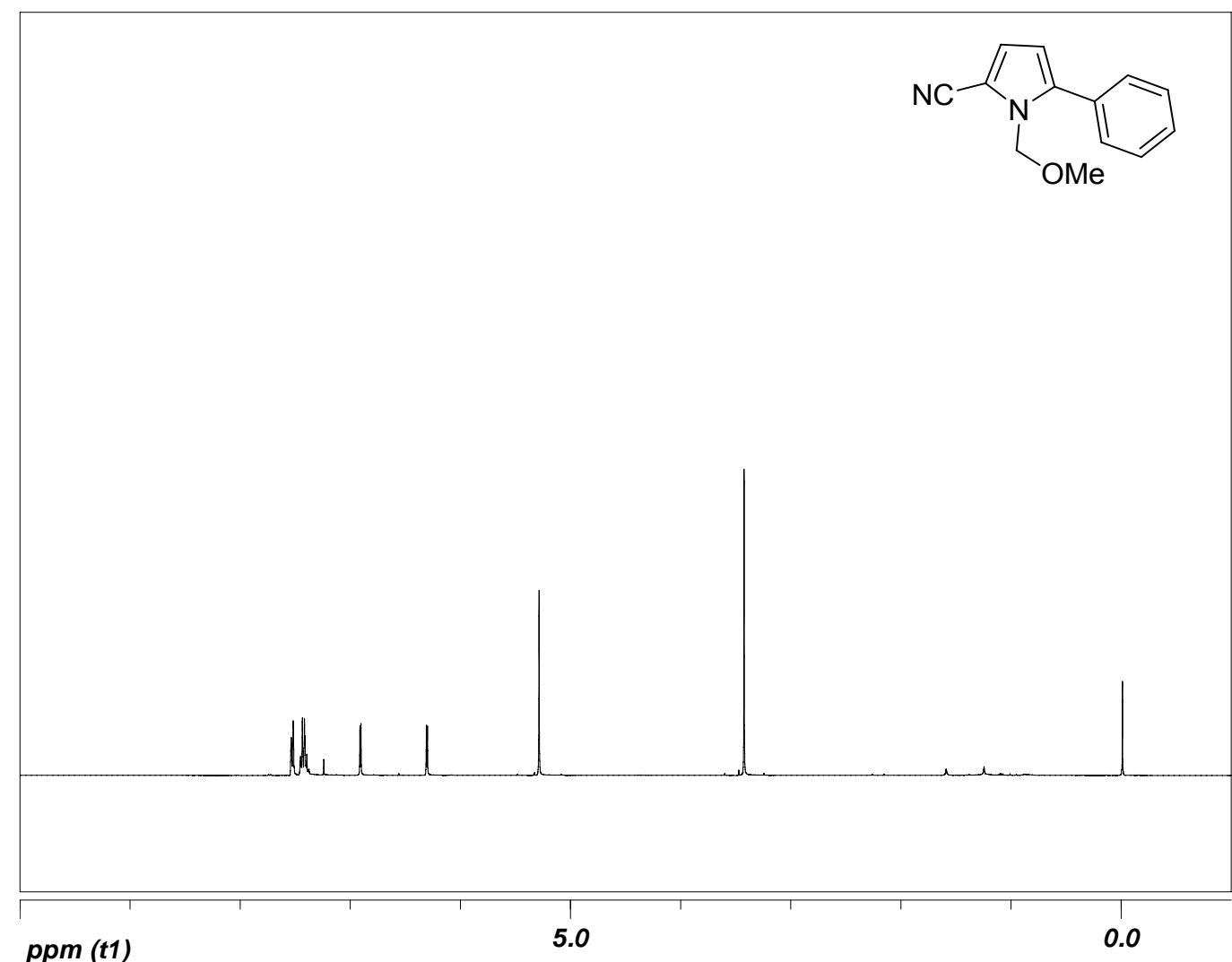

ppm (t1)

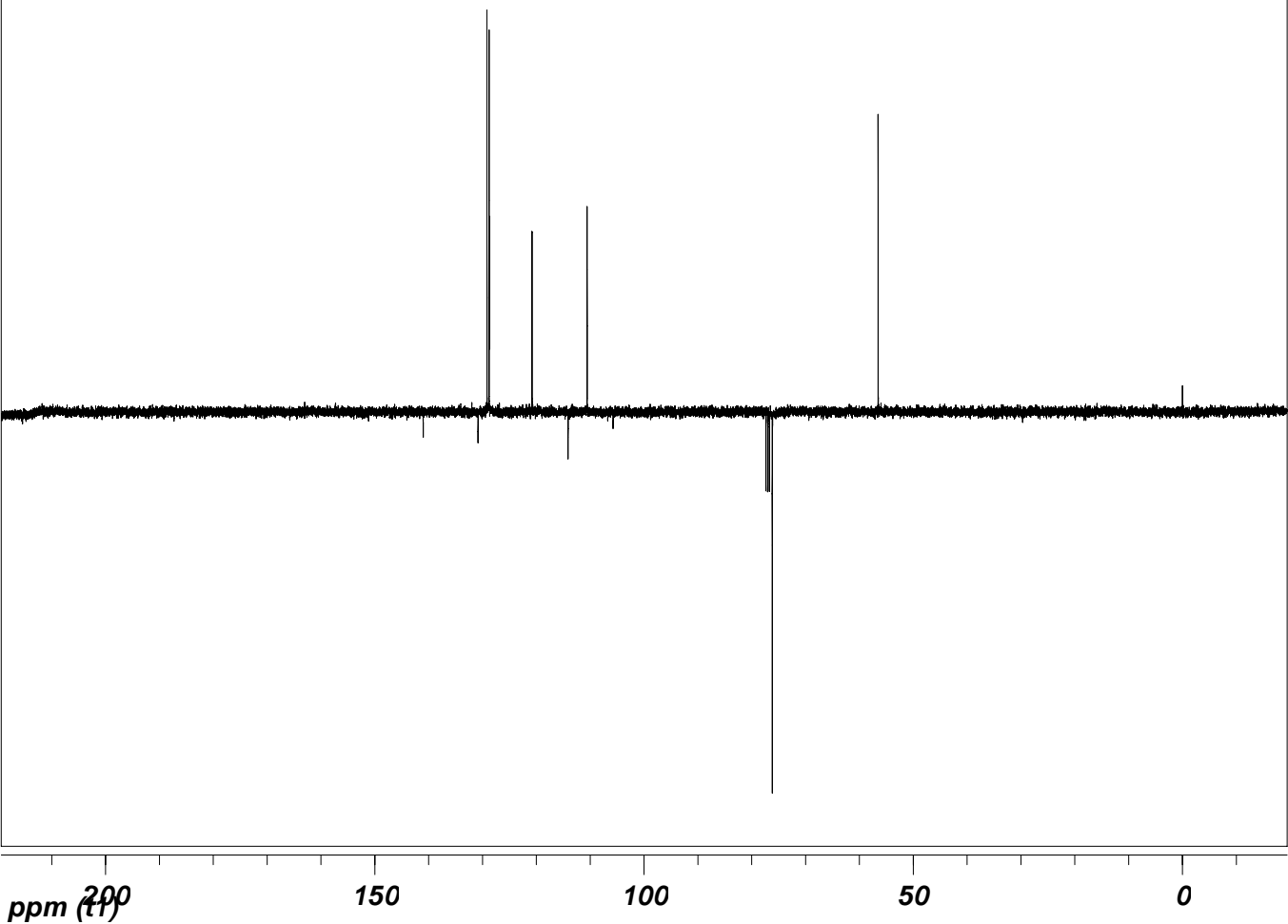



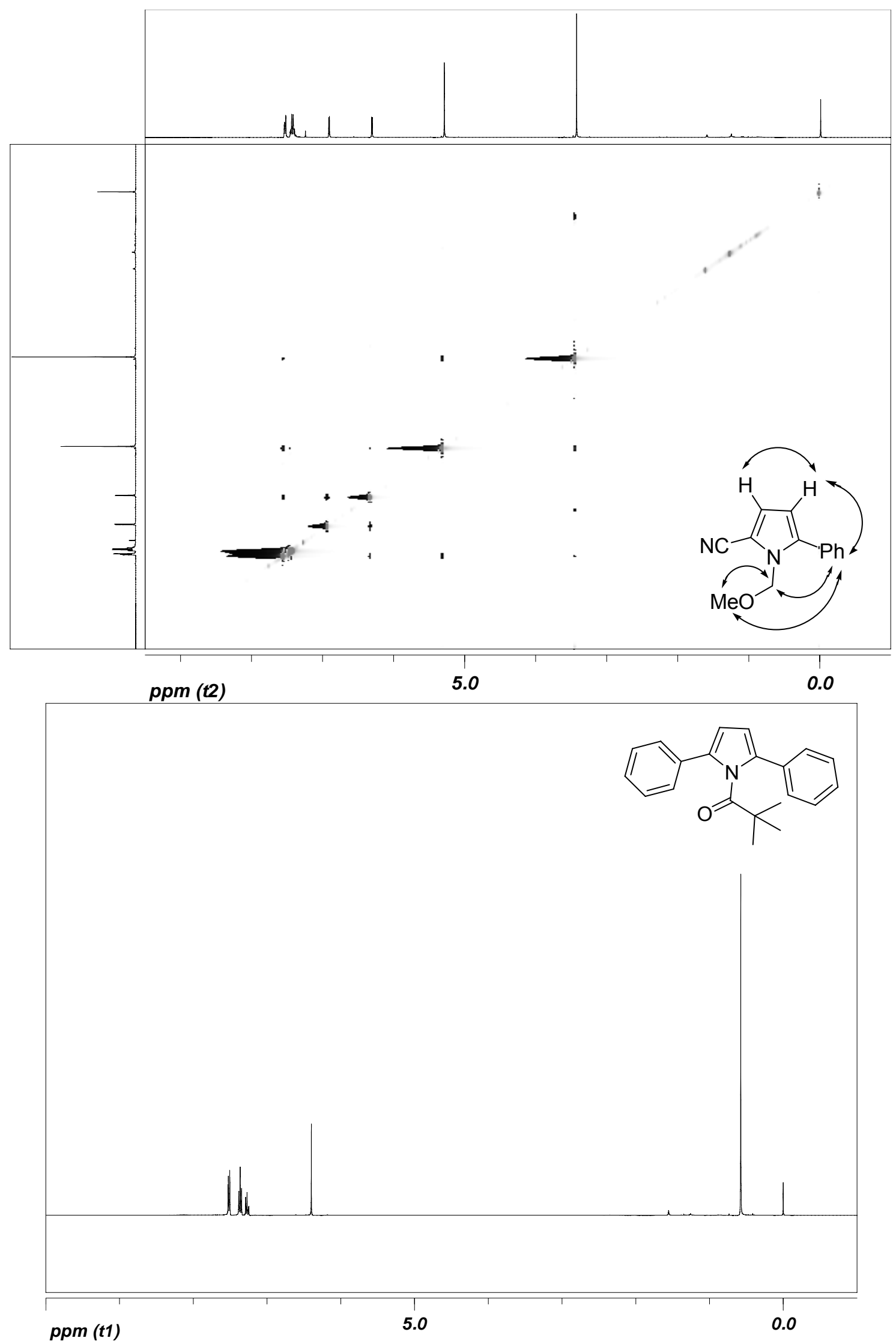


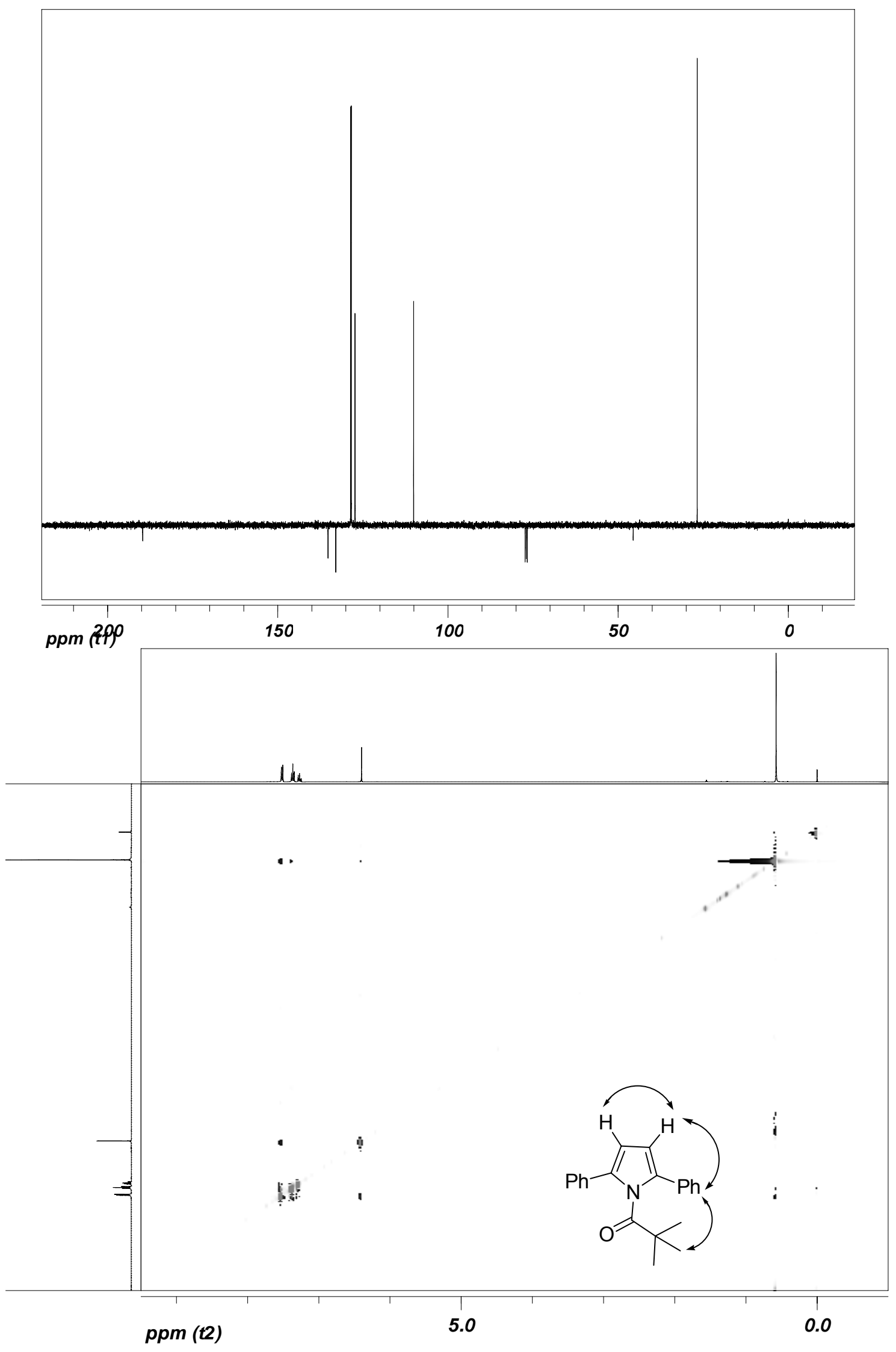

